\title{
Investigation of Kronecker-based \\ Recovery in Compressive Sensing
}

\author{
by \\ Dipayan Mitra

\begin{abstract}
A thesis submitted to the
Faculty of Graduate Studies and Research

in partial fulfillment of the requirements for the degree of

Master of Applied Science in Electrical and Computer Engineering
\end{abstract}

Department of Systems and Computer Engineering

Carleton University

Ottawa, Ontario, Canada

(C)2019, Dipayan Mitra 


\section{Abstract}

In this thesis, modified Kronecker-based compressive sensing (CS) 1-D and 2-D recovery techniques with random and deterministic measurement matrices are investigated to improve signal quality despite resource restricted acquisition. For regular recovery of individual segments of the compressed signal, the measurement and sparsifying matrices are required. While the regular Kronecker-based CS recovery technique uses expanded Kronecker measurement and basis matrices to achieve one-time recovery of a collection of compressively acquired segmented signals, in the proposed modified Kronecker-based CS recovery, a new basis matrix, which is an expanded and dense version of the original basis matrix, is used. The reduction of mutual coherence between the expanded Kronecker measurement and the expanded basis matrix leads to improvement in the recovery of the signal. Deterministic sensing further improves the recovery and preserves the structure of the acquired signal in the compressed domain which can be exploited for compressed domain signal processing algorithms. 


\section{Acknowledgements}

I would like to express my deep and sincere gratitude to the people who have helped me in the completion of the thesis.

Firstly, I would begin by thanking my supervisor Prof. Sreeraman Rajan for his guidance throughout the course of my M.A.Sc. Prof. Rajan and his wife have been welcoming and extremely supportive during the past two years of my stay in Ottawa. It has been a privilege to work with Prof. Rajan, who patiently spent countless hours discussing problems and guiding me to understand research methods and find solutions.

I would like to extend my gratitude towards Mr. Hadi Zanddizari, who has been extremely supportive in proving research materials and exchanging ideas. Mr. Zanddizari has taken personal efforts in my research by being a co-author for a number of publications with me.

I thank Dr. Bhashyam Balaji for sharing IR image database and providing insights into signal processing techniques, which has lead to a significant contribution of my thesis. I would also like to thank Prof. Martin Bouchard and Prof. Ioannis Lambadaris, whose teaching helped me to obtain a background enabling the completion of this thesis.

I would like to thank my friends, Mr. Rajshekhar Mukherjee, Mr. Elliot Yeung, Mr. Shanmugaraja K.V, Mr. Mohamed Abdelazez, Ms. Behnaz Fakhar, Mr. Aly 
Khedr, Mr. Zachary Baird who helped me in all possible ways. I would also like to thank NSERC \& Carleton University for supporting the work and last but not the least my parents for their unwavering support. 


\section{Contents}

1 Thesis in a Nutshell 1

1.1 Summary of the Thesis . . . . . . . . . . . . . . 1

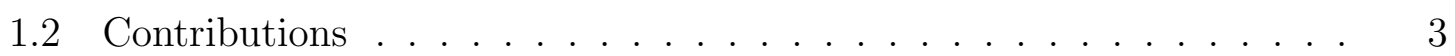

1.3 Organization of the Thesis $\ldots \ldots \ldots \ldots \ldots \ldots$

2 Compressive Sensing $\quad 6$

2.1 Introduction . . . . . . . . . . . . . . . . 6

2.2 Mathematical Formulation of CS $\ldots \ldots \ldots \ldots \ldots$

2.3 Design of Measurement Matrix _ . . . . . . . . . . . 9

2.4 Classification of Measurement Matrices . . . . . . . . . . . 10

2.4.1 Random Measurement Matrix . . . . . . . . . . . . . . . 11

2.4.2 Deterministic Measurement Matrix . . . . . . . . . . . . 12

2.5 Sparse Reconstruction . . . . . . . . . . . . . . . . . . . 14

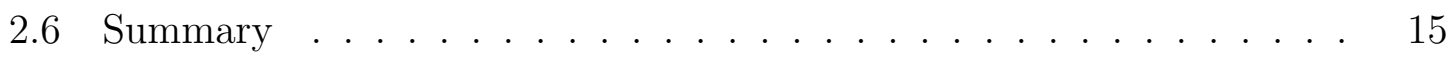

3 Kronecker-based Compressive Sensing Recovery 17

3.1 Introduction $\ldots \ldots \ldots \ldots \ldots \ldots \ldots \ldots \ldots \ldots \ldots \ldots \ldots \ldots$

3.2 Kronecker Product . . . . . . . . . . . . . . . . . . . . 18

3.3 Kronecker-based Compressive Measurement Matrix Expansion . . . . 19 
3.3.1 Kronecker-based Measurement Matrix Expansion . . . . . . . 20

3.4 Standard Kronecker-based Recovery . . . . . . . . . . . . . . . . . . . 21

3.5 Modified Kronecker-based CS Recovery . . . . . . . . . . . . 23

3.6 Summary . . . . . . . . . . . . . . . . . . 27

4 Kronecker-based Compressive Recovery for 1-D Signal 28

4.1 Introduction . . . . . . . . . . . . . . . . . . . . . . . . . . . 29

4.2 Segmentation-based Compressive ECG Measurement Techniques . . . 31

4.2.1 Advantages ......................... 31

4.2 .2 Disadvantages . . . . . . . . . . . . . . . . . 32

4.3 Way out? . . . . . . . . . . . . . . . . . . 32

4.4 Random Measurement Framework . . . . . . . . . . . . . . . . 33

4.5 Discussions . . . . . . . . . . . . . . . . . . . . . . . 34

4.5.1 Advantages ....................... 34

4.5.2 Disadvantages . . . . . . . . . . . . . . . . 34

4.6 Deterministic Measurement Framework . . . . . . . . . . . . . . . . 35

4.7 Problem Formulation and Kronecker-based Recovery . . . . . . . . 36

4.7.1 Segmentation-based ECG Signal Representation . . . . . . . . 36

4.7.2 Kronecker-based Measurement Matrix Formation for recovery 37

4.7.3 Segmented Measurement Vector Generation and Matrix Rep-

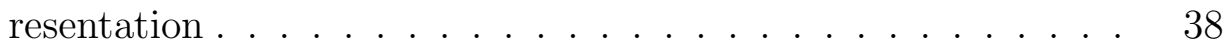

4.7.4 Kronecker-based Recovery of Segmented ECG Signals . . . . . 39

4.8 Performance Evaluation Metrics . . . . . . . . . . . . . . . . . . . . 44

4.8.1 Compression Ratio (CR) . . . . . . . . . . . . . 45

4.8.2 Percentage Root Mean square Difference (PRD) . . . . . . . . 45

4.8.3 Normalized Percentage Root Mean square Difference (PRDN) 45 
4.8.4 Root Mean Square (RMS) Error . . . . . . . . . . . . . . 46

4.8.5 Zero-th lag of Cross-Correlation (CC) . . . . . . . . . 46

4.8 .6 Quality Score $(\mathrm{QS}) \ldots \ldots \ldots$. . . . . . . . . . 46

4.8.7 Maximum Amplitude Error (MAX) . . . . . . . . . . 47

4.9 Database Description . . . . . . . . . . . . . . . . . . 47

4.10 Results and Analysis . . . . . . . . . . . . . . . 47

4.10 .1 Impact of Wavelet Decomposition Levels . . . . . . . . . . 48

4.10 .2 Impact of CR . . . . . . . . . . . . . . . . 52

4.10.3 Analysis of Quality of Reconstruction of Compressed ECG Signals 53

4.10.4 Recovery of Noisy ECG: Choice of Measurement Framework . 57

4.11 Summary . . . . . . . . . . . . . . . 60

5 Kronecker-based Compressive Recovery for 2-D Signal 62

5.1 Introduction . . . . . . . . . . . . . . . 63

5.2 Column-wise 2 -D CS . . . . . . . . . . . . . . . . . . 64

5.3 Segmentation-based CS . . . . . . . . . . . . . . . . . . . . 64

5.3.1 Segmentation-based 2-D Measurement . . . . . . . . . 66

5.4 Performance Evaluation Metrics . . . . . . . . . . . . . . 67

5.4 .1 Signal-to-Noise Ratio . . . . . . . . . . . . . . . . 67

5.4 .2 Structural Similarity Index _ . . . . . . . . . . . 67

5.5 Database Selection . . . . . . . . . . . . . . . . . 68

5.6 Results and Analysis . . . . . . . . . . . . . . . . . . . . . 69

5.7 Summary of column-wise $2-\mathrm{D}$ CS $\ldots \ldots \ldots \ldots \ldots$

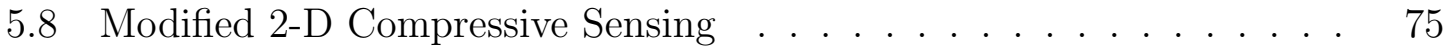

5.8 .1 Recovery in Modified 2-D CS . . . . . . . . . . 77

5.9 Performance Analysis . . . . . . . . . . . . . . . . . . . . . . . . 78 
5.9.1 Peak Signal-to-Noise Ratio (PSNR) _ . . . . . . . . 78

$5.9 .2 \quad$ Structural Similarity Index $(\mathrm{SSIM}) \ldots \ldots \ldots$

5.10 Database Selection . . . . . . . . . . . . . . . . . 79

5.11 Compression Ratio $(\mathrm{CR}) \ldots \ldots \ldots \ldots \ldots$

5.12 Result and Analysis . . . . . . . . . . . . . . . . . 80

5.13 Summary . . . . . . . . . . . . . . . . . 83

6 Deterministic Sensing-Based Compressed Domain Image Analysis 84

6.1 Introduction . . . . . . . . . . . . . . 85

6.2 2-D Deterministic Measurement and Recovery . . . . . . . . . 86

6.3 Feature-Based Template Matching . . . . . . . . . . . . . . 87

6.4 Database Selection . . . . . . . . . . . . . . . . . . . . . . . 89

6.4.1 Low Resolution Image $\ldots \ldots \ldots$

6.4 .2 Medium Resolution Image $\ldots \ldots \ldots$

6.4 .3 High Resolution Image . . . . . . . . . . . . . . . . . . 90

6.5 Results and Analysis . . . . . . . . . . . . . . 90

6.5.1 Structural Similarity Analysis _. . . . . . . . . . . . . 91

6.5.2 Compressed Domain Feature Preservation _. . . . . . . . 92

6.6 Summary . . . . . . . . . . . . . . . . . . . . 97

7 Conclusions, Limitations and Future Works $\quad 99$

7.1 Limitations and Scope of Future Works . . . . . . . . . . . . . 101

$\begin{array}{ll}\text { Appendices } & 104\end{array}$

$\begin{array}{ll}\text { References } & 114\end{array}$ 


\section{List of Figures}

2.1 Signal acquisition technique in CS . . . . . . . . . . . 8

2.2 Classification of $\Phi$ based on structure . . . . . . . . . . . . . . 11

2.3 Linear filter-based block diagram representation of DBBD measurement matrix . . . . . . . . . . . . . . . . . . . . . 13

2.4 Block diagram representation of sparse reconstruction. . . . . . . . 15

3.1 Block diagram representation of the standard Kronecker-based recovery technique. . . . . . . . . . . . . . . . . 22

3.2 Block diagram representation of the modified Kronecker-based recovery

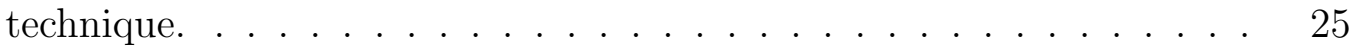

4.1 Signal segmentation into $p$ number of smaller blocks. . . . . . . . . . 31

4.2 Block diagram representation of random measurement framework. . . 33

4.3 Block diagram representation of deterministic measurement framework. 36

4.4 Comparison between different decomposition levels of Daubechies-15 wavelet at $\mathrm{CR}=50 \%$ with $\boldsymbol{\Phi}=$ DBBD. . . . . . . . . . . . . . 49

4.5 Comparison between different decomposition levels of Symlets-8 wavelet at $\mathrm{CR}=50 \%$ with $\boldsymbol{\Phi}=$ DBBD. . . . . . . . . . . . . . . . 49 
4.6 Comparison between different decomposition levels of different wavelets at $\mathrm{CR}=50 \%$ with $\boldsymbol{\Phi}=\mathrm{DBBD}$ using the modified Kronecker-based recovery technique. . . . . . . . . . . . . . . .

4.7 Comparison between different decomposition levels of different wavelets at $\mathrm{CR}=50 \%$ with $\boldsymbol{\Phi}=\mathrm{DBBD}$ using the standard Kronecker-based recovery technique. ....................... 50

4.8 Reconstruction quality comparison on for various levels of compression using $\boldsymbol{\Psi}=$ Biorthogonal-3.3 wavelet basis with $\boldsymbol{\Phi}=$ DBBD. (A) Comparison of SNR as a function of CR. (B) Comparison of Quality Score (QS) as a function of CR. . . . . . . . . . . . . .

4.9 Reconstruction quality comparison on for various levels of compression using $\Psi=$ Biorthogonal-3.3 wavelet basis and $\boldsymbol{\Phi}$ as a random matrix with values drawn from normal distribution. (A) Comparison of SNR as a function of CR. (B) Comparison of Quality Score (QS) as a function of CR. . . . . . . . . . . . . . .

4.10 Reconstruction quality comparison on for various levels of compression using $\boldsymbol{\Psi}=$ Biorthogonal-3.3 wavelet basis and $\boldsymbol{\Phi}$ as a random matrix with values drawn from Bernoulli distribution. (A) Comparison of SNR as a function of CR. (B) Comparison of Quality Score (QS) as a function of CR. . . . . . . . . . . . . . . .

4.11 Visual representation of ECG signals. Recovery has been performed using SL0 algorithm, $\boldsymbol{\Phi}=\mathrm{DBBD}$ and $\mathrm{CR}=50 \%$ with $\boldsymbol{\Psi}=$ DCT. (A) Original signal (x). (B) Recovered signal ( $\hat{\mathbf{x}})$. (C) Reconstructed signal quality assessment based on RMS error. . . . . . . . . . . . 56 
4.12 Visual representation of ECG signals in presence of $-15 \mathrm{~dB}$ noise at source (before compression). Recovery has been performed using SL0 algorithm, $\mathbf{\Phi}=\mathrm{DBBD}$ and $\mathrm{CR}=75 \%$ with $\boldsymbol{\Psi}=$ Coiflets-5. (A) Original signal $(\mathbf{x})$. (B) Signal corrupted by $-15 \mathrm{~dB}$ additive noise $\left(\mathrm{x}^{\prime}\right)$. (C) Recovered signal ( $\hat{\mathbf{x}})$. (D) Reconstructed signal quality assessment based on RMS Error. . . 58

4.13 Visual representation of ECG signals in presence of $-15 \mathrm{~dB}$ noise at source (before compression). Recovery has been performed using SL0 algorithm, $\boldsymbol{\Phi}=$ Random matrix with values chosen from Bernoulli distribution and $\mathrm{CR}=75 \%$ with $\Psi=$ Coiflets-5. (A) Original signal (x). (B) Signal corrupted by $-15 \mathrm{~dB}$ additive noise $\left(\mathbf{x}^{\prime}\right)$. (C) Recovered signal $(\hat{\mathbf{x}})$. (D) Reconstructed signal quality assessment based on RMS Error. . . . . . . . . . . . . . . . .

5.1 Column-wise measurement and recovery of 2-D signals. . . . . . . . . 65

5.2 Non-segmented image with each column of length $N$. . . . . . . 65

5.3 Column-wise segmented image with each segment of length $n$. . . . 66

5.4 Reconstruction quality comparison between segmentation-based CS and ordinary CS methods. . . . . . . . . . . . . . . 70

5.5 Analysis using DCT as sparsifying dictionary for $\mathrm{CR}=50 \%$ : (a) Original MR Image, (b) Recovered by the modified Kronecker-based CS recovery technique $(\mathrm{SNR}=38.99 \mathrm{~dB}),(\mathrm{c})$ Recovered by the standard Kronecker-based CS recovery technique $(\mathrm{SNR}=31.31 \mathrm{~dB}) \ldots . . . . \quad 71$

5.6 Analysis using DCT as sparsifying dictionary for $\mathrm{CR}=93.75 \%$ : (a) Original MR Image, (b) Recovered by the modified Kronecker-based CS recovery technique $(\mathrm{SNR}=23.05 \mathrm{~dB}),(\mathrm{c})$ Recovered by the standard Kronecker-based CS recovery technique $(\mathrm{SNR}=20.74 \mathrm{~dB})$. . . 
5.7 Comparison using structural similarity index for $\mathrm{CR}=93.75 \% \ldots \ldots$. . 74

5.8 Modified 2-D CS: row \& column-wise measurement and recovery. . . . 77

5.9 Block diagram representation of the modified 2-D CS recovery. . . . . 78

5.10 Comparison using structural similarity index for $\mathrm{CR}=75 \%$. . . . . 80

5.11 Comparison using structural similarity index for $\mathrm{CR}=87.5 \%$. . . . 81

5.12 Comparison using structural similarity index for $\mathrm{CR}=93.75 \%$. . . . 81

5.13 Analysis using DCT as sparsifying dictionary at $\mathrm{CR}=93.75 \%$ for the modified 2-D CS: (a) Original MR Image, (b) Recovered by the modified Kronecker-based CS recovery technique $(\mathrm{PSNR}=34.89 \mathrm{~dB})$, (c) Recovered by the standard Kronecker-based CS recovery technique $(\mathrm{PSNR}=28.51 \mathrm{~dB}) \ldots \ldots \ldots \ldots \ldots$

6.1 Visual representation of images at different compression levels. (A) Original image, (B) Image with $\mathrm{CR}=50 \%$, (C) Image with $\mathrm{CR}=75 \% .93$

6.2 Feature-based template matching from compressed infra-red images. (A) Original image compressed at $\mathrm{CR}=50 \%$. (B) Template generated from uncompressed image. (C) Outlier removed match point identification using SURF algorithm. (D) Bounding box indicating the position of the template in the compressed image. . . . . . . . . . . .

6.3 Feature-based template matching from compressed medium resolution images. (A) Original image compressed at $\mathrm{CR}=50 \%$. (B) Template generated from uncompressed image. (C) Outlier removed match point identification using SURF algorithm. (D) Bounding box indicating the position of the template in the compressed image. . . . . . . . . 
6.4 Feature-based template matching from compressed high resolution images. (A) Original image compressed at $\mathrm{CR}=50 \%$. (B) Template generated from uncompressed image. (C) Outlier removed match point identification using SURF algorithm. (D) Bounding box indicating the position of the template in the compressed image. . . . . . . . . . . 96 


\section{List of Tables}

4.1 Statistical analysis of recovery performance using the modified Kronecker-based technique: $\mathrm{CR}=50 \%, \boldsymbol{\Phi}=\mathrm{DBBD} \ldots \ldots . . . . .51$

4.2 Analysis of reconstruction quality using the standard and the modified Kronecker-based technique for $\mathrm{CR}=50 \%$. . . . . . . . . . 55

6.1 Structural Similarity Analysis (Compressed Images) . . . . . . . . . . 92

6.2 Structural Similarity Analysis (Recovered Images) . . . . . . . . . . . 92

1 Reconstruction Quality Analysis: Case 1 . . . . . . . . . . . 105

2 Reconstruction Quality Analysis: Case 2 . . . . . . . . . . . . 105

3 Reconstruction Quality Analysis: Case 3 . . . . . . . . . . . . . 105

4 Reconstruction Quality Analysis: Case 4 . . . . . . . . . . . . 106

$5 \quad$ Reconstruction Quality Analysis: Case 5 . . . . . . . . . . . . . 106

6 Reconstruction Quality Analysis: Case 6 . . . . . . . . . . . . 106

7 Reconstruction Quality Analysis: Case 7 . . . . . . . . . . . 106

8 Reconstruction Quality Analysis: Case 8 . . . . . . . . . . . 106

$9 \quad$ Reconstruction Quality Analysis: Case 9 . . . . . . . . . . . . . 107

10 Reconstruction Quality Analysis: Case 10 . . . . . . . . . . . . . 107

11 Reconstruction Quality Analysis (Modified 2-D CS): Case 1 . . . . . 107

12 Reconstruction Quality Analysis (Modified 2-D CS): Case 2 . . . . 107 
13 Reconstruction Quality Analysis (Modified 2-D CS): Case 3 . . . 107

14 Reconstruction Quality Analysis (Modified 2-D CS): Case 4 . . . . . 108

15 Reconstruction Quality Analysis (Modified 2-D CS): Case 5 . . . . . 108

16 Reconstruction Quality Analysis (Modified 2-D CS): Case 6 . . . . . 108

17 Reconstruction Quality Analysis (Modified 2-D CS): Case 7 . . . . . 108

18 Reconstruction Quality Analysis (Modified 2-D CS): Case 8 . . . . . 108

19 Reconstruction Quality Analysis (Modified 2-D CS): Case 9 . . . . . 109

20 Reconstruction Quality Analysis (Modified 2-D CS): Case 10 . . . . . 109

21 Reconstruction Quality Analysis (Modified 2-D CS with Random (Normal) Matrix): Case 1 . . . . . . . . . . . . . . . . . 109

22 Reconstruction Quality Analysis (Modified 2-D CS with Random (Nor-

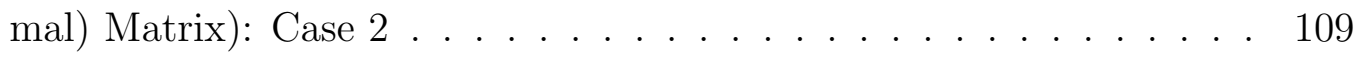

23 Reconstruction Quality Analysis (Modified 2-D CS with Random (Normal) Matrix): Case 3 . . . . . . . . . . . . . . . . . 109

24 Reconstruction Quality Analysis (Modified 2-D CS with Random (Normal) Matrix): Case 4 . . . . . . . . . . . . . . . . . 110

25 Reconstruction Quality Analysis (Modified 2-D CS with Random (Normal) Matrix): Case 5 . . . . . . . . . . . . . . . 110

26 Reconstruction Quality Analysis (Modified 2-D CS with Random (Normal) Matrix): Case 6 . . . . . . . . . . . . . . . . . 110

27 Reconstruction Quality Analysis (Modified 2-D CS with Random (Normal) Matrix): Case 7 . . . . . . . . . . . . . . . . . 110

28 Reconstruction Quality Analysis (Modified 2-D CS with Random (Nor-

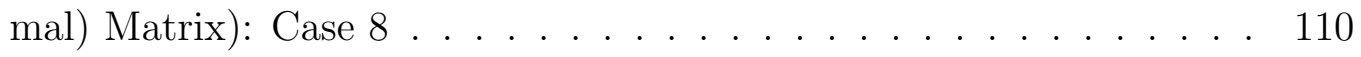

29 Reconstruction Quality Analysis (Modified 2-D CS with Random (Nor-

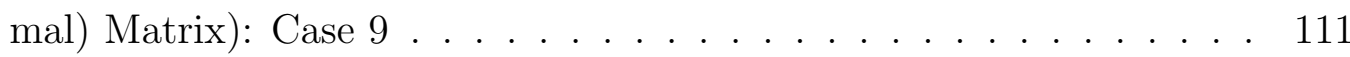


30 Reconstruction Quality Analysis (Modified 2-D CS with Random (Normal) Matrix): Case 10 . . . . . . . . . . . . . . . 111

31 Reconstruction Quality Analysis (Modified 2-D CS with Random (Bernoulli) Matrix): Case 1 . . . . . . . . . . . . . 111

32 Reconstruction Quality Analysis (Modified 2-D CS with Random (Bernoulli) Matrix): Case 2 . . . . . . . . . . . . . . . . . 111

33 Reconstruction Quality Analysis (Modified 2-D CS with Random (Bernoulli) Matrix): Case 3 . . . . . . . . . . . . . . . 111

34 Reconstruction Quality Analysis (Modified 2-D CS with Random (Bernoulli) Matrix): Case 4 . . . . . . . . . . . . . 112

35 Reconstruction Quality Analysis (Modified 2-D CS with Random (Bernoulli) Matrix): Case 5 . . . . . . . . . . . . . . . 112

36 Reconstruction Quality Analysis (Modified 2-D CS with Random (Bernoulli) Matrix): Case 6 . . . . . . . . . . . . . . . . . 112

37 Reconstruction Quality Analysis (Modified 2-D CS with Random (Bernoulli) Matrix): Case 7 . . . . . . . . . . . . . 112

38 Reconstruction Quality Analysis (Modified 2-D CS with Random (Bernoulli) Matrix): Case 8 . . . . . . . . . . . . . . . . 112

39 Reconstruction Quality Analysis (Modified 2-D CS with Random (Bernoulli) Matrix): Case 9 . . . . . . . . . . . . . . . 113

40 Reconstruction Quality Analysis (Modified 2-D CS with Random (Bernoulli) Matrix): Case 10 . . . . . . . . . . . . 113 


\title{
List of Abbreviations
}

\author{
BP Basis Pursuit \\ CoSaMP COmpressive SAmpling Matching Pursuit \\ CS Compressive Sensing \\ CVD Cardiovascular Disease \\ DBBD Deterministic Binary Block Diagonal Matrix
}

DoG Difference of Gaussian

ECG Electrocardiogram

GP Gradient Pursuit

LASSO Least Absolute Shrinkage and Selection Operator

MRI Magnetic Resonance Imaging

MSE Mean Square Error

NCD Noncommunicable Disease

OMP Orthogonal Matching Pursuit 
PCA Principal Component Analysis

PRD Percentage Root Mean square Difference

PSNR Peak Signal-to-Noise Ratio

SL0 Smooth $\ell^{0}$

SNR Signal-to-Noise Ratio

StOMP Stagewise Orthogonal Matching Pursuit

SURF Speeded-up Robust Features

UAV Unmanned Aerial Vehicle

WBAN Wireless Body Area Networks

\section{Nomenclature}

* Convolution operation

$<., .>$ Inner product operation

$[.]^{T} \quad$ Matrix transpose operation

$\downarrow$ Decimation operation

$\delta_{k} \quad$ Isometric constant

Ł.」 $\quad$ Floor function

$\Phi \quad$ Measurement matrix 
$\Psi \quad$ Sparsifying basis

I Identity matrix

X Boldfaced upper-case letters denote matrices

x Boldfaced lower-case letters denote vectors

$\mu \quad$ Mutual coherence (Exception: In Chapter 6, $\mu$ represents mean of a distribution)

$\otimes \quad$ Kronecker product operation

$\phi_{A, B}(0)$ Zero-th lag of cross-correlation between A \& B

$\Re \quad$ Reconstruction algorithm

$\sigma \quad$ Standard deviation (Exception: In Chapter 6, $\sigma$ represents standard scalespace, i.e. convolution of a 2-D image with Gaussian kernel)

$\Theta \quad$ Total dictionary

$\|\cdot\|_{p} \quad \ell_{p}$ norm

$\|\cdot\|_{\max }$ Max norm

var Variance of a distribution (Only for Chapter 6)

$\operatorname{supp}($.$) Support function$ 


\section{Chapter 1}

\section{Thesis in a Nutshell}

\subsection{Summary of the Thesis}

In recent times with the advent of 'data explosion' continuous acquisition of signals has become the need of the hour. However, the data analysis (or processing) is often performed in a 'processing on demand' fashion. As a result, most of the sensors designed for continuous signal acquisition are connected to a remote system where the collected signals are transmitted for further processing. Applications of such systems can be found in a wide range of areas, from healthcare to surveillance cameras in airports or public places. With almost every sensor connected to the internet, the need for efficient sampling schemes where the number of samples is reduced as much as possible or compression schemes for data transfer where only the needed amount of data is transferred. Compressive sensing is an appropriate choice for both cases.

Compressive sensing (CS) relies on the compressibility of the signal in order to acquire it at a lower rate. As most of the connected devices do not have large onboard storage memory or computational memory space, smaller segments of signals are acquired. These compressed measurements which are obtained in smaller seg- 
ments are sent to a remote unit for recovery. As the recovery of the original signal from compressed measurement requires a lot of computation, such computationally demanding operations are outsourced to clouds which do not have resource restrictions. Often, clouds are also used for storing the acquired compressed signals and recovery is performed 'on demand'.

In this thesis, a novel recovery technique is investigated to improve the quality of the reconstructed signals. The underlying assumption in this thesis is the measurements are obtained using compressive sensing of an already acquired discrete signal. This compression is done in order to transmit the measurement to a remote resourcerich location, such as a cloud, where on-demand reconstruction would be performed. If the analog signal is acquired through compressive sensing technique, say in an IoT device, then the compressively acquired measurements may be transferred to a remote location for reconstruction on demand. In either case, the recovery process follows the same mathematical formulation. Improvement in recovery offered by the Kronecker-based CS recovery technique is theorized and recovery improvements are demonstrated by compressing and recovering 1-D ECG signals obtained from the MIT-BIH Arrhythmia database. The choice of measurement matrix and its role in the proposed recovery technique is investigated. In particular, random and deterministic measurement matrices are used in this thesis. The influence of sparsifying basis in determining the quality of the recovered signal is also investigated for 1-D ECG signals.

The 1-D Kronecker-based CS recovery technique is then extended for 2-D signals by considering 2-D signals as a collection of columns of 1-D signals. A novel 2-D CS technique (row \& column-wise CS) is proposed and its effectiveness is compared against the extended 1-D compression and recovery techniques. A deterministic 2-D compressive sensing that preserves the aspect ratio and the morphology of the 2-D 
signals is also considered in this thesis. The deterministic 2-D compressive sensing and recovery technique is applied on images of high, medium and low resolution. As the deterministic compressive sensing preserves the structure and morphology of the original signal in the compressed domain, it is possible to conduct further signal processing in the compressed domain without the need for any recovery.

\subsection{Contributions}

The contributions of the thesis are as follows,

1. Development of a modified Kronecker-based recovery technique for deterministic compressive sensing for $1-\mathrm{D}$ and $2-\mathrm{D}$ signals.

2. Comparison of deterministic and random compressive sensing frameworks for signal quality improvement using a modified Kronecker-based recovery technique.

3. Development of a novel 2-D aspect ratio preserving CS technique and application of the modified Kronecker-based recovery technique for 2-D signals.

4. Demonstration of structure and morphology preservation through 2-D deterministic aspect ratio preserving compressive sensing leading to signal processing in the compressed domain without the need for any recovery.

5. Investigation of Kronecker-based CS recovery technique for ECG signals using various sparsifying dictionaries, measurement matrices and noise levels for various compression levels.

The following list of journal and conference publications have resulted from this thesis: 
- Journal Publications

1. D. Mitra, H. Zanddizari and S. Rajan, "Investigation of Kronecker-based Recovery of Compressed ECG Measurements," under revision after first submission to IEEE Transactions on Instrumentation and Measurement, 2019 .

2. H. Sadreazami, D. Mitra, S. Rajan and M. Bolic, "Fall Detection in Compressed Domain using Machine Learning, 2019. (Under Preparation).

- Conference Publications

1. D. Mitra, S. Rajan and B. Balaji, "Deterministic compressive sensing approachfor compressed domain image analysis," in 2019 IEEE Sensors Applications Symposium (SAS), pp. 1-6, Sophia Antipolis, France, March 2019 .

2. D. Mitra, H. Zanddizari and S. Rajan, "Improvement of recovery in segmentation-based parallel compressive sensing, in 2018 IEEE International Symposium on Signal Processing and Information Technology (ISSPIT), pp. 501-506, Lousville, USA, Dec 2018.

3. D. Mitra, H. Zanddizari and S. Rajan, "Improvement of signal quality during recovery of compressively sensed ECG signals," in 2018 IEEE International Symposium on Medical Measurements and Applications (MeMeA), pp. 1-5, Rome, Italy, June 2018.

\subsection{Organization of the Thesis}

In Chapter 2, mathematical formulations of CS has been discussed along with different choice of measurement matrices and recovery (reconstruction) techniques. Chapter 3 
introduces Kronecker-based CS. Application of Kronecker-based CS in 1-D signal has been investigated in Chapter 4. Random and deterministic measurement frameworks are provided and role played by random and deterministic matrices on the quality of reconstruction is investigated. This chapter also presents a quantitative analysis of Kronecker-based CS recovery technique for varying compression ratios, sparsifying basis. Chapter 5 illustrates the extension of the 1-D Kronecker-based CS recovery technique to 2-D signals. A novel 2-D CS approach is introduced and investigated in this chapter. Chapter 5 presents a comparative study of the proposed and the extended recovery techniques for 2-D signals. Possibility of compressed domain signal processing, based on an aspect ratio preserving deterministic sensing framework, by eliminating the need to perform CS reconstruction, is introduced in Chapter 6. The thesis is concluded in Chapter 7 along with suggestions for future work. Results obtained during the course of this thesis work is presented in Appendices 1 to 40. 


\section{Chapter 2}

\section{Compressive Sensing}

\section{$2.1 \quad$ Introduction}

In modern data-driven society, countless signal acquisition techniques exist based on canonical sampling theory proposed by Claude Elwood Shannon [1]. A significant part of the data acquired by traditional Shannon-Nyquist sampling rate "can be thrown away', without any 'perceptual loss' [2]. In other words, the signal can be compressed. The process of acquiring signals at a high sampling rate and later 'throwing away' is highly inefficient. To avoid throwing away of samples, in [2] Donoho proposed a sensing technique exploiting the 'compressibility' of the signals. In this technique, only an adequate number of samples required for representing the signal in the domain of a compact representation are acquired. Many signals such as bio-signals, natural images or audio signals are typically 'compressible' on a certain basis, such as wavelets [3].

Compressive Sensing (CS) is a signal processing technique that ensures nearperfect signal reconstruction (with high probability), from samples acquired significantly lower than the Shannon-Nyquist sampling rate, for a large class of signals hav- 
ing a sparse representation in certain predetermined basis. In compressive sensing, a signal is compressed and sampled simultaneously. CS may be considered as a linear transformation, where the signal lying in a higher dimensional space is projected onto a lower dimensional space. The result of the transformation is called measurement. Compressive sensing-based acquisition of analog signal leads to several advantages in the hardware such as reduced size and power. If the analog signal is acquired compressively, then a natural question arises, 'Did the transformation preserve all the information in the original analog signal?'. This question is answered through the reconstruction or recovery process. Several recovery techniques exist in the literature, the details of which are presented later in this chapter. Unlike compressive sensing of analog signal, in this thesis, the measurements are obtained using compressive sensing of an already acquired discrete signal. This compression is done in order to transmit the measurement to a remote resource-rich location such as a cloud where reconstruction may be performed. If the analog signal is acquired through compressive sensing technique, say in an IoT device, then the compressively acquired measurements need to be transferred to a remote location for reconstruction on demand. In either case, the recovery process follows the same mathematical formulation.

\subsection{Mathematical Formulation of CS}

Any discrete time signal $\mathbf{x} \in \mathbb{R}^{N}$ can be represented in terms of orthonormal basis as follows:

$$
\mathbf{x}_{N \times 1}=\mathbf{\Psi}_{N \times N} \mathbf{s}_{N \times 1}
$$

Here $\boldsymbol{\Psi}_{N \times N}$ is termed as sparsifying matrix or sometimes as the dictionary of the signal $\mathbf{x}$. In the theory of CS, $\mathbf{s}$ is called a $k$-sparse or just sparse vector if in $\mathbf{s}$ there exists $k$ non-zero elements and $(N-k)$ are strictly zero elements, with $k<<N$. 
Either the sparsifying matrix $\Psi$ is assumed to be known a priori or needs to be determined. In literature, a number of adaptive algorithms have been proposed to find the sparse representation of compressible signals [4-10]. However, in this thesis, the sparsifying matrix is assumed to be already predetermined. Often, in practice, signals are not strictly sparse, where strictly only $k$ components of $\mathbf{s}$ are non-zeros and it will be assumed that $(N-k)$ components are negligible when compared to the other $k$ elements of s. Such signals are called 'compressible signals'. In this thesis, the term compressible and sparse will be used interchangeably.

Now let $\mathbf{x} \in \mathbb{R}^{N}$ be a ( $k$-sparse) signal having a sparse representation in a basis $\Psi_{N \times N}$. The measurement vector $\mathbf{y}$ is obtained from the signal $\mathbf{x}$ through a linear transformation as shown below:

$$
\mathbf{y}_{M \times 1}=\mathbf{\Phi}_{M \times N} \mathbf{x}_{N \times 1}
$$

where $k<M<<N$ and $\mathbf{y} \in \mathbb{R}^{M}$. Here $\boldsymbol{\Phi}_{M \times N}$ is called the sensing or measurement or projection matrix. Substituting equation 2.1 into equation 2.2 we can write the following:

$$
\mathbf{y}_{M \times 1}=\boldsymbol{\Phi}_{M \times N} \mathbf{\Psi}_{N \times N} \mathbf{s}_{N \times 1}
$$

Oftentimes, in literature the product of $\boldsymbol{\Phi}_{M \times N} \boldsymbol{\Psi}_{N \times N}$ is called as $\boldsymbol{\Theta}_{M \times N}$ or total dictionary. Figure 2.1 shows the block diagram representation of CS-based signal acquisition technique.

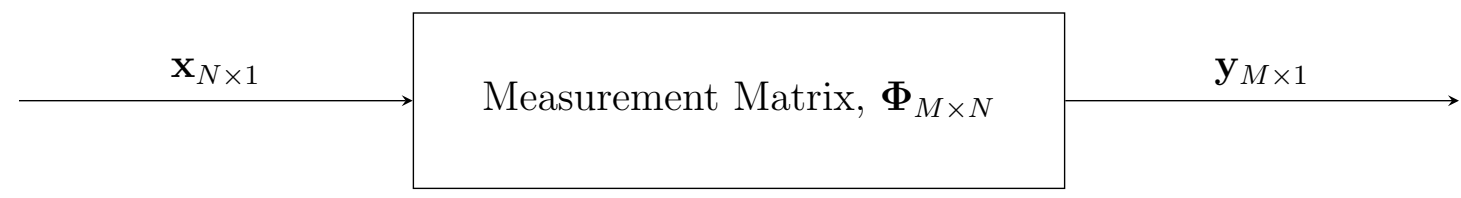

Figure 2.1: Signal acquisition technique in CS 
Once the measurement vector is formed, the measurement vector can be transferred to a remote location and 'on demand' recovery may be performed or recovered given the measurement vector and the measurement and sparsifying matrices. One of the major challenges in CS lies in the design of $\boldsymbol{\Phi}_{M \times N}$, which ensures a successful recovery. The properties that a measurement matrix must satisfy in order to have a good recovery or reconstruction is discussed in the following section.

\subsection{Design of Measurement Matrix}

The key consideration behind the design of a measurement matrix, $\boldsymbol{\Phi}_{M \times N}$, is to preserve the information content of the $k$-sparse signal $\mathbf{x}_{N \times 1}$. If a priori information regarding the positions of the $k$ non-zero elements are available, $\boldsymbol{\Phi}_{M \times N}$ can be designed for solving a least-square problem by forming the $M \times k$ measurement matrix by choosing the columns of $\boldsymbol{\Phi}_{M \times N}$ pertaining to the positions of non-zero elements in $\mathbf{x}$. However, in a practical measurement scenario, it is highly unlikely that such $a$ priori position information would be available. It becomes an NP-hard combinatorial problem when it comes to choosing $k$ columns of the measurement matrix when the positions of the sparse elements of $\mathbf{x}$ are not known. By not explicitly identifying the $k$ non-zero locations of the $\mathbf{x}$, mathematicians have identified solutions by reposing the recovery problem as a convex optimization problem. In order to ensure that a solution to the CS recovery problem, Candès proposed Restricted Isometry Property (RIP) for measurement matrices [11]. $\boldsymbol{\Phi}_{M \times N}$ for a $k$-sparse signal $\mathbf{x}_{N \times 1}$ is said to maintain RIP of order $k$ if the following inequality holds:

$$
\left(1-\delta_{k}\right) \leq \frac{\|\mathbf{\Phi} \mathbf{x}\|_{2}^{2}}{\|\mathbf{s}\|_{2}^{2}} \leq\left(1+\delta_{k}\right)
$$


where $\|\cdot\|_{2}$ denotes the $\ell_{2}$ norm and $\delta_{k} \in(0,1)$ is the isometric constant. Although RIP is a sufficient condition to ensure reconstruction of the $k$-sparse signal from the measurement $\mathbf{y}_{M \times 1}$, there exists no polynomial time algorithm to verify RIP. As a result, it is very complex to check RIP condition by solving an NP-complete problem in a practical measurement scenario. Alternatively, in literature, the relationship between two matrices $\boldsymbol{\Phi}_{M \times N}$ and $\boldsymbol{\Psi}_{N \times N}$ is exploited. Based on the principal of CS, $\mathbf{x}_{N \times 1}$ cannot have sparse representation in both $\boldsymbol{\Phi}_{M \times N}$ and $\boldsymbol{\Psi}_{N \times N}$ [12]. In other words, $\boldsymbol{\Phi}_{M \times N}$ needs to be incoherent with $\boldsymbol{\Psi}_{N \times N}$. Hence, mutual coherence $(\mu)$ between $\boldsymbol{\Phi}_{M \times N}$ and $\boldsymbol{\Psi}_{N \times N}$ is chosen to be an alternative to RIP and defined as follows $[13,14]$ :

$$
\mu(\boldsymbol{\Phi}, \boldsymbol{\Psi})=\max _{i \neq j}\left|<\tilde{\Phi}_{i}, \Psi_{j}>\right|
$$

where $\tilde{\boldsymbol{\Phi}}=\boldsymbol{\Phi}^{T}$ and $<., .>$ denotes inner product operation between the column

vectors $\tilde{\Phi}_{i}, \Psi_{j} \in \mathbb{R}^{N}$ of $\boldsymbol{\Phi}^{T}$ (row of $\boldsymbol{\Phi}$ ) and $\boldsymbol{\Psi}$. For the total dictionary, $\boldsymbol{\Theta}_{M \times N}$, a very small value of $\mu$ ensures near perfect signal reconstruction from the compressive measurement $\mathbf{y}_{M \times 1}$.

For a chosen $\Psi$, an appropriate measurement matrix needs to be designed that has a low mutual coherence with $\Psi$. Researchers have proposed several measurement matrices that satisfy the mutual coherence requirement. The following section summarizes the different options for the choice of measurement matrices.

\subsection{Classification of Measurement Matrices}

Measurement matrices can be classified into two main categories: random measurement matrix and deterministic measurement matrix. Figure 2.2 presents the classification of each category of matrices [15]. 


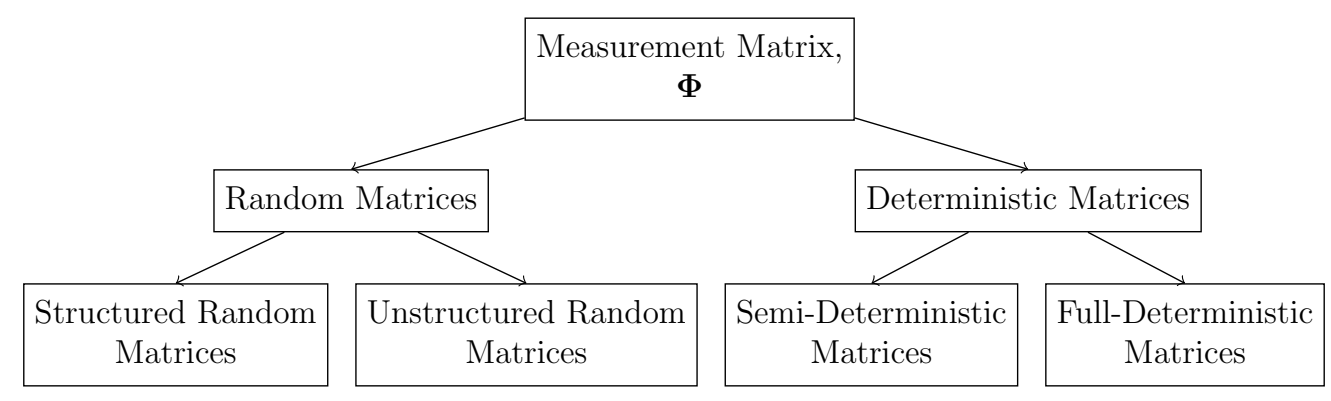

Figure 2.2: Classification of $\boldsymbol{\Phi}$ based on structure

\subsubsection{Random Measurement Matrix}

Baraniuk et al. showed that the random matrices, with elements drawn from various distributions, maintain previously discussed RIP property [16]. As a result, with high probability, random matrices guarantee near perfect signal reconstruction. Random matrices with independent and identically distributed (i.i.d) elements drawn from Gaussian $\mathcal{N}\left(0, \frac{1}{M}\right)$, sub-Gaussian and Bernoulli distribution $\left( \pm \frac{1}{\sqrt{M}}\right)$ are two unstructured random sensing matrices, known to satisfy RIP with overwhelming probability [17]. Based on studies focused on the symmetry and asymptotic properties of random matrices, numerous structured random matrices have been designed to meet the purpose of compressive measurement [18]. Numerous design techniques of structured random matrices have been introduced in [19-25]. Despite maintaining RIP, i.e. guaranteeing near perfect signal reconstruction with high probability, random measurement matrices suffer from the following two major disadvantages:

1. In order to recover the signal from the compressive measurement $\mathbf{y}_{M \times 1}$, a priori knowledge about measurement matrix is required by the sparse recovery algorithm. Hence, the random measurement matrix is required to be stored. For most of the practical measurement scenario, storage of the measurement matrix for signal recovery as well as further realization is not feasible. 
2. Based on the properties of random measurement matrices, signal recovery is guaranteed with a probabilistic notion; hence, obtaining the best possible reconstruction matrix is not guaranteed since measurement may also contain measurement noise.

To circumvent the shortcomings of the random measurement matrices, deterministic sensing is preferred for most of the practical measurement applications. One advantage of random measurement matrix is the compression and encoding can be achieved simultaneously. When privacy is an issue, use of the random matrix is preferred over deterministic matrices. However, this thesis will not address privacy issues related to data security.

\subsubsection{Deterministic Measurement Matrix}

To meet the objective of maintaining RIP and avoiding the drawbacks of the random measurement matrices, numerous design techniques have been proposed for deterministic sensing. $\mathrm{Yu}$ et al. proposed a deterministic measurement matrix construction technique by forming a Toeplitz-structured matrix from chaotic sequence [26]. In $[27,28]$ circular deterministic measurement matrices are constructed. Both the circular and Toeplitz-structured deterministic matrices are classified as semi-deterministic matrices. Utilizing chirp-functions, Applebaum et al. introduced deterministic measurement matrix construction [29]. Howard et al. utilized second-order Reed Muller code to generate deterministic measurement matrix, which maintains RIP property [30]. In [31], BCH codes were used to construct deterministic measurement matrices. In literature, a number of deterministic construction techniques have been proposed [32-34]. Aforementioned class of deterministic measurement matrices are known as full-deterministic matrices. 
Among many full-deterministic matrix construction techniques, in this thesis, an emphasis on the construction of deterministic binary block diagonal (DBBD) matrix, introduced in [35], is put. Construction of a DBBD matrix of size $M \times N$ can be viewed as a shift-invariant linear low-pass filter followed by a decimation operation. Decimation operation is carried out with decimation by a factor of $\left\lfloor\frac{N}{M}\right\rfloor$. Figure 2.3 shows the block diagram representation of DBBD measurement matrix.

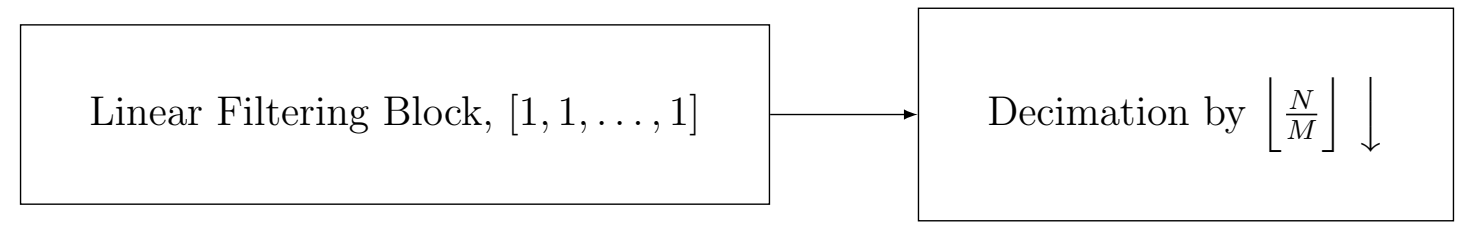

Figure 2.3: Linear filter-based block diagram representation of DBBD measurement matrix

Equation 2.6 is an example of DBBD measurement matrix for $M=4$ and $N=16$. Evidently, in each row there exits $\frac{N}{M}=4$ number of 1's. Similarly deterministic matrix construction can be performed for various combinations of $N$ and $M$.

$$
\mathbf{\Phi}_{4 \times 16}=\left[\begin{array}{llll}
{[1111]} & & & \\
& {[1111]} & & \\
& & {[1111]} & \\
& & & \\
& & &
\end{array}\right]
$$

DBBD measurement matrix is considered in this thesis for the following reasons:

1. DBBD measurement matrix is easy-to-implement and hence suitable for hardware implementation.

2. DBBD matrix can be generated without any storage space requirement. Moreover, the number of multiplications needed to be performed is 0 . In comparison, generation of most of the random matrices require $M \times N$ multiplications [35]. 
3. Since DBBD matrix is linear filtering followed by decimation, morphology and features of the signal remain intact in the compressed domain. It lends easily to the development of compressed domain signal analysis and possible development of signal processing algorithms for compressed domain signals without the need for reconstruction.

\subsection{Sparse Reconstruction}

Once the signal is measured compressively and sent to a remote unit for storage and recovery on-demand, the challenge in CS is to recover the sparse vector $\mathbf{s}_{N \times 1}$ from the measurement $\mathbf{y}_{M \times 1}$, whenever required. The problem of finding $\mathbf{s}_{N \times 1}$ can be formulated based on $\ell_{0}$ minimization in the following way:

$$
\hat{\mathbf{s}}=\arg \min \|\mathbf{s}\|_{0} \quad \text { s.t. } \quad \mathbf{y}=\boldsymbol{\Phi} \Psi \mathbf{s}
$$

where $\|.\|_{0}$ represents $\ell_{0}$ norm. But, such a formulation is NP-hard and too complex to solve. For measurements $M=k \log \left(\frac{N}{k}\right)<<N$ acquired by measurement matrices maintaining RIP, solution based on $\ell_{1}$-minimization ensures sparse reconstruction [36]. $\ell_{1}$-minimization problem to recovery of $\mathbf{s}_{N \times 1}$ can be represented by equation 2.8 .

$$
\hat{\mathbf{s}}=\arg \min \|\mathbf{s}\|_{1} \quad \text { s.t. } \quad \mathbf{y}=\boldsymbol{\Phi} \Psi \mathbf{s}
$$

where $\|.\|_{1}$ represents $\ell_{1}$ norm. This is to be noted that both the minimization problems require a priori knowledge of $\boldsymbol{\Phi}$ and $\boldsymbol{\Psi}$. Figure 2.4 represents the block diagram of the sparse reconstruction technique stated above.

Over the years, a number of sparse recovery algorithms have been proposed, based 


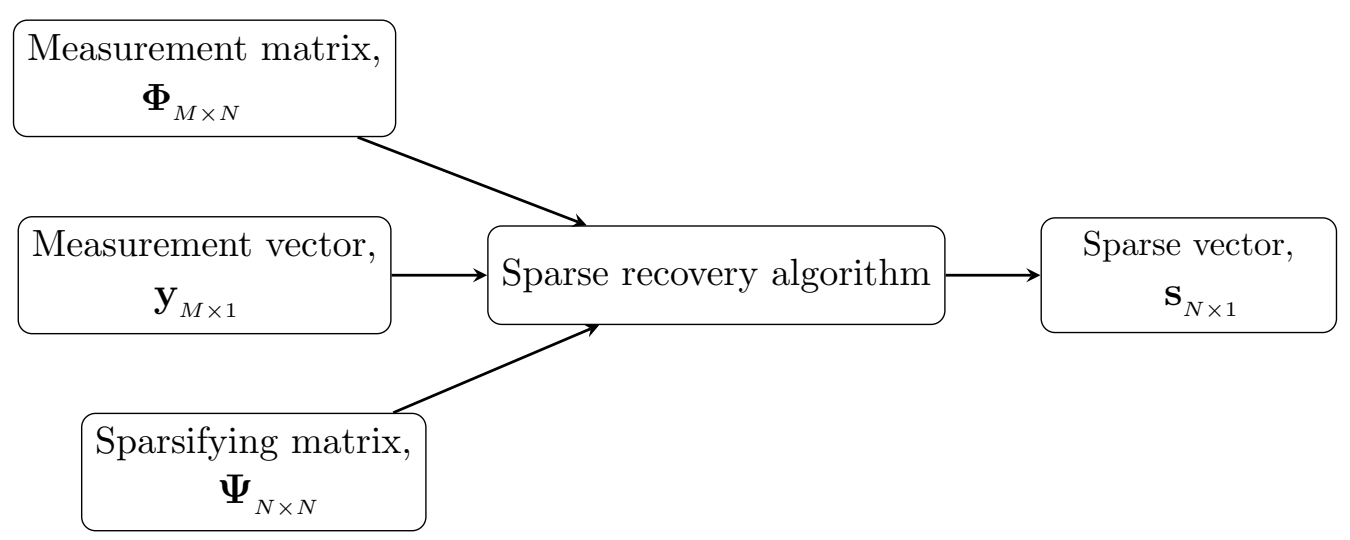

Figure 2.4: Block diagram representation of sparse reconstruction.

on convex optimization-based solution. Algorithms like BP [37], LASSO [38], Gradient Projection [39], can be classified into the aforementioned category. Greedy pursuit-based techniques, such as OMP [40-42], CoSaMP [43], StOMP [44], provide an alternative to convex optimization-based approach. Selection of a particular recovery algorithm is application specific. A classification of recovery algorithms based on particular signals have been discussed by Marques et al. in [45]. In [46], Mohimani et al. proposed a recovery technique based on smoothed $\ell_{0}$-norm. In this thesis, $\ell_{0}$-norm-based recovery technique known as SL0 is used as preferred sparse reconstruction algorithm, because of its faster execution time over other algorithms.

\subsection{Summary}

In this chapter, a brief introduction to compressive measurement of a signal is presented. Properties of measurement matrices are briefly discussed and classification of measurement matrices based on its structure is explained. Limitations of random measurement matrices as well as the construction and properties of DBBD measurement matrix have also been discussed. The chapter also provides a brief introduction to sparse reconstruction and algorithms available for compressive recovery. In the 
next chapter of the thesis, Kronecker CS, its properties, benefits in terms of signal quality improvement and potential applications will be discussed. 


\section{Chapter 3}

\section{Kronecker-based Compressive Sensing Recovery}

\subsection{Introduction}

This chapter presents the background for Kronecker-based CS recovery. Design criteria of the measurement matrices, discussed in Chapter 2 for traditional CS, would be extended in light of Kronecker product-based techniques. Effect on key properties for designing measurement matrices, such as RIP and $\mu$, would be investigated in the context of Kronecker-based CS recovery. Advantages and applications of such recovery schemes would also be discussed. A modified Kronecker-based CS recovery technique will be presented. The improvement of signal quality in signal recovery by applying such techniques will be discussed. The chapter will conclude with a mathematical proof for improvement in recovered signal obtained using the modified Kronecker-based technique. 


\subsection{Kronecker Product}

Definition 3.2.1. The Kronecker product of two matrices $\mathbf{P}_{a_{1} \times b_{1}}$ and $\mathbf{Q}_{a_{2} \times b_{2}}$ is defined as,

$$
\mathbf{R}_{a_{1} a_{2} \times b_{1} b_{2}}:=\mathbf{P}_{a_{1} \times b_{1}} \otimes \mathbf{Q}_{a_{2} \times b_{2}}
$$

where $\otimes$ denotes the Kronecker product between two matrices $\mathbf{P}$ and $\mathbf{Q}$. Following shows an example of the Kronecker product between two matrices:

$$
\left[\begin{array}{cccc}
p_{1,1} & p_{1,2} & \ldots & p_{1, b_{1}} \\
p_{2,1} & p_{2,2} & \ldots & p_{2, b_{1}} \\
& & \ddots & \\
p_{a_{1}, 1} & p_{a_{1}, 2} & \ldots & p_{a_{1}, b_{1}}
\end{array}\right] \otimes \mathbf{Q}:=\left[\begin{array}{cccc}
p_{1,1} \mathbf{Q} & p_{1,2} \mathbf{Q} & \ldots & p_{1, b_{1}} \mathbf{Q} \\
p_{2,1} \mathbf{Q} & p_{2,2} \mathbf{Q} & \ldots & p_{2, b_{1}} \mathbf{Q} \\
& & \ddots & \\
& & & \\
p_{a_{1}, 1} \mathbf{Q} & p_{a_{1}, 2} \mathbf{Q} & \ldots & p_{a_{1}, b_{1}} \mathbf{Q}
\end{array}\right]
$$

Equation 3.1 can be extended to represent the Kronecker product between two vectors $\mathbf{p}_{a_{1} \times 1}$ and $\mathbf{q}_{a_{2} \times 1}$ as follows:

$$
\mathbf{r}_{a_{1} a_{2} \times 1}:=\mathbf{p}_{a_{1} \times 1} \otimes \mathbf{q}_{a_{2} \times 1}
$$

Durate et al. introduced Kronecker-based CS for many applications, such as compressive measurement of multidimensional signals (particularly 2D signals) and measurement techniques in distributed sensing environments [47]. Kronecker-CSbased measurement allows effective 'multistage CS acquisition' for enabling faster and efficient sensing. Unlike Durate's work, in this thesis, Kronecker-based recovery or reconstruction is carried out while the compression phase is carried out using a single measurement matrix. In the consecutive sections of this thesis, improvement 
of signal quality during recovery of the signals using Kronecker-based method will be discussed.

In this thesis, measurement is done 'independently' while the recovery is done 'jointly'. In order to do the joint recovery, the measurement matrix is expanded using the original measurement matrix employed to obtain the measurements in the compression phase. It may be noted that the selection of sparsifying basis does not have any such constraint. As the sparsifying basis is required to be used only during the joint recovery process, two possible embodiment can be utilized. In the first embodiment, the sparsifying basis corresponding to the original measurement size may be considered. As in the joint recovery, the original matrix is expanded, the sparsifyin basis is also expanded. This is called the "standard Kronecker-based recovery' in this thesis. In the second embodiment, a new bigger sparsifying basis is generated corresponding to the size of the expanded measurement matrix. This is called the 'modified Kronecker-based recovery' in this thesis. These techniques are explained in the following sections.

\subsection{Kronecker-based Compressive Measurement Matrix Expansion}

In Chapter 2, the properties of measurement matrix that ensure signal reconstruction, have been discussed. Such properties, RIP and $\mu$, should remain unchanged as a result of Kronecker product-based expansion of measurement matrix. 


\subsubsection{Kronecker-based Measurement Matrix Expansion}

Assume that a single measurement matrix $\boldsymbol{\Phi}$ is used to measure $p$ portions of the signal. Let us suppose each individual measurement vector, so obtained, be denoted by $\mathbf{y}_{i}: i=1,2, \ldots, p$. Concatenating $p$ individual measurement vectors, the resultant measurement vector can be formed. Equation 3.4 shows the aforementioned concatenated resultant measurement vector $\hat{\mathbf{y}}$.

$$
\hat{\mathbf{y}}=\left[\begin{array}{c}
\mathbf{y}_{1} \\
\mathbf{y}_{2} \\
\vdots \\
\mathbf{y}_{p}
\end{array}\right]
$$

This concatenation is equivalent to obtaining the measurement using a single measurement matrix $(\mathbf{\Phi})$ expanded by Kronecker product and the resultant matrix $(\hat{\boldsymbol{\Phi}})$ given below by the equation 3.5,

$$
\hat{\boldsymbol{\Phi}}=\mathbf{I}_{p \times p} \otimes \boldsymbol{\Phi}=\left[\begin{array}{cccc}
\boldsymbol{\Phi} & 0 & \ldots & 0 \\
0 & \Phi & \ldots & 0 \\
& & \ddots & \\
0 & 0 & \ldots & \Phi
\end{array}\right]
$$

where, $\mathbf{0}$ represents matrices of appropriate sizes having all elements equal to 0 . Please note that the diagonal of $\hat{\boldsymbol{\Phi}}$ consists of $p$ number of $\boldsymbol{\Phi}$ matrices.

In order for the resultant measurement matrix to ensure recovery, the RIP property should be maintained. In [47], Durate et al. showed the RIP of $\hat{\boldsymbol{\Phi}}$ would remain unchanged. Isometric constant $\delta_{k}$ (introduced in Chapter 2 - Section 2.3) is intrinsically connected to the singular values of individual $\Phi$ that are used in the construction 
of $\hat{\boldsymbol{\Phi}}$. Based on this, bounds of RIP has been presented in [48]. The isometric constant of the orthonormal matrix, $\mathbf{I}_{p \times p}$ is zero and based on the derivation given in [47], the Kronecker product-based expansion of the measurement matrix will have the same RIP as the original measurement matrix.

\subsection{Standard Kronecker-based Recovery}

In order to recover the sparse signal acquired as $p$ segments all at once, the measurement matrix is expanded using Kronecker product as shown above. In the regular CS recovery, the measurement matrix used in the measurement phase will be considered. There is a freedom to choose the sparsifying basis. If a signal is sparse in many bases, one has the choice to use a sparsifying basis that has the lowest mutual coherence with the measurement matrix. Likewise, in the standard Kronecker-based recovery, one has the freedom to choose the sparsifying basis. Since the measurement matrix has been expanded using the Kronecker product, the sparsifying matrix will also need to be expanded using the Kronecker product. Using a single sparsifying basis $\boldsymbol{\Psi}$, the resultant expanded sparsifying dictionary is given by equation 3.6 :

$$
\hat{\Psi}=\mathbf{I}_{p \times p} \otimes \Psi=\left[\begin{array}{cccc}
\Psi & 0 & \ldots & 0 \\
0 & \Psi & \ldots & 0 \\
& & \ddots & \\
0 & 0 & \ldots & \Psi
\end{array}\right]
$$

where, $\mathbf{0}$ represents matrices of appropriate size having all elements equal to 0 . Please note that the diagonal of $\hat{\boldsymbol{\Psi}}$ consists of $p$ number of $\boldsymbol{\Psi}$ matrices.

Once $\hat{\mathbf{y}}, \hat{\boldsymbol{\Phi}}$ and $\hat{\boldsymbol{\Psi}}$ are available, the sparse solution is obtained using one of the reconstruction algorithms stated in Section 2.5 of the previous chapter. Figure 3.1 
shows the block diagram of the standard Kronecker-based recovery technique.

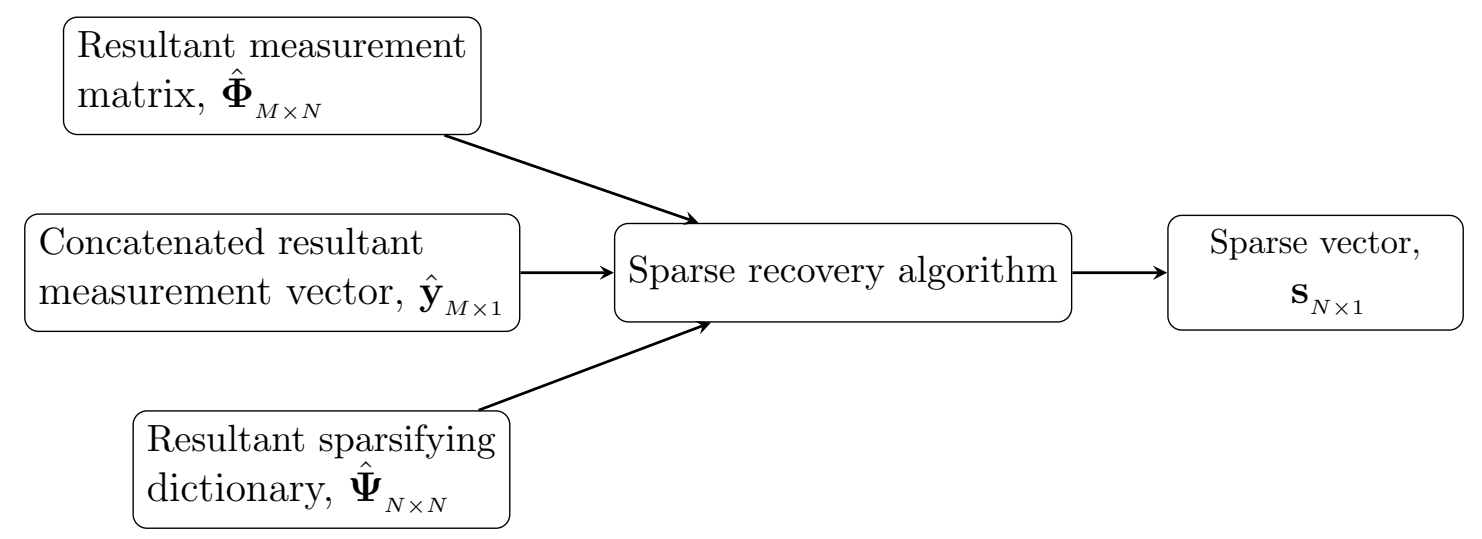

Figure 3.1: Block diagram representation of the standard Kronecker-based recovery technique.

Based on the discussion of Chapter 2 - Section 2.3, preservation of $\mu$ determines the quality of the reconstruction.

The following observations are in order:

- Since $\mu$ does not change due to Kronecker expansion, the recovery quality is the same as the standard CS recovery.

- Even though Kronecker method is employed, recovery is done once but not jointly. Each segment is recovered independently of another.

- When a un-segmented signal is compressed and recovered, the reconstructed signal will be superior to that obtained through the standard Kronecker-based technique. Because of segmentation, mutual coherence $\mu$ increases, leading to inferior recovery.

To obtain a better recovered signal when signals are segmented and compressed, a modified Kronecker-based approach is investigated in this thesis. 


\subsection{Modified Kronecker-based CS Recovery}

Based on the discussions of the previous section, the reduction in the size of $\boldsymbol{\Phi}$ and $\boldsymbol{\Psi}$ leads to poor recovery. Reduction in size of $\boldsymbol{\Phi}$ is unavoidable as the measurement is performed in smaller segments. Since recovery is performed in remote systems with no dearth for computational capacity, generation of $\boldsymbol{\Psi}$ in larger size is not a concern. Let us consider the standard Kronecker-based technique, where a signal $\mathbf{x}_{N \times 1}$ is segmented into $p$ smaller segments, each of length $N^{\prime}\left(N^{\prime}<N\right)$. These individual segments are then compressed using a measurement matrix, $\boldsymbol{\Phi}_{M^{\prime} \times N^{\prime}}$. Let the individual $p$ segments be sparse when $\boldsymbol{\Psi}_{N^{\prime} \times N^{\prime}}$ is used as the sparsifying dictionary. Since the concatenated measurement vector is considered for recovery, both the measurement and the sparsifying matrices will be expanded in the recovery phase using Kronecker product as given respectively by the following equations 3.7 and 3.8 :

$$
\begin{gathered}
\hat{\boldsymbol{\Phi}}_{M \times N}=\mathbf{I}_{p \times p} \otimes \boldsymbol{\Phi}_{M^{\prime} \times N^{\prime}} \\
\hat{\boldsymbol{\Psi}}_{N \times N}=\mathbf{I}_{p \times p} \otimes \boldsymbol{\Psi}_{N^{\prime} \times N^{\prime}}
\end{gathered}
$$

Instead of expanding the sparsifying basis, in the modified Kronecker-based technique a new basis, $\Psi_{N \times N}^{\prime}$, is regenerated from the same sparsifying basis by expanding the basis to $N^{\prime} \times N^{\prime}$. The newly formed orthonormal sparsifying matrix is dense unlike the one used in the standard Kronecker-based recovery. Equation 3.9 shows the newly formed sparsifying basis of size $N \times N$. 


$$
\boldsymbol{\Psi}_{N \times N}^{\prime}=\left[\begin{array}{cccc}
\boldsymbol{\Psi}_{1,1}^{\prime} & \boldsymbol{\Psi}_{1,2}^{\prime} & \ldots & \boldsymbol{\Psi}_{1, p}^{\prime} \\
\boldsymbol{\Psi}_{2,1}^{\prime} & \boldsymbol{\Psi}_{2,2}^{\prime} & \ldots & \boldsymbol{\Psi}_{2, p}^{\prime} \\
& & \ddots & \\
\boldsymbol{\Psi}_{p, 1}^{\prime} & \boldsymbol{\Psi}_{p, 2}^{\prime} & \ldots & \boldsymbol{\Psi}_{p, p}^{\prime}
\end{array}\right]
$$

where each individual matrices $\boldsymbol{\Psi}_{i, j}^{\prime}, \forall i, j=1,2, \ldots, p$, are of size $N^{\prime} \times N^{\prime}$. Note that the sparsifying basis $\Psi_{N \times N}^{\prime}$, i.e. matrix of size $N \times N\left(N=p \times N^{\prime}\right)$, is regenerated from the same basis (the basis used in the standard Kronecker-based technique) and $\Psi_{i, j}^{\prime}$ 's are smaller sub-matrices of size $N^{\prime} \times N^{\prime}$.

In order to explain the difference between the expanded sparsifying basis $\hat{\Psi}$ (for the standard Kronecker-based recovery technique) and the regenerated sparsifying basis $\Psi^{\prime}$ (for the modified Kronecker-based recovery technique), an example with DCT dictionary is considered. In this example, let us suppose $N^{\prime}=2$ and $p=2$. Hence, $\boldsymbol{\Psi}_{2 \times 2}$ can be expanded to form $\hat{\boldsymbol{\Psi}}_{4 \times 4}$ in the following way:

$$
\hat{\mathbf{\Psi}}_{4 \times 4}=\mathbf{I}_{2 \times 2} \otimes \mathbf{\Psi}_{2 \times 2}=\left(\begin{array}{ccc|cc}
\hline 0.7071 & 0.7071 & 0 & 0 \\
0.7071 & -0.7071 & 0 & 0 \\
\hline 0 & 0 & 0.7071 & 0.7071 \\
0 & 0 & 0.7071 & -0.7071
\end{array}\right)
$$

where each section highlighted with $(\square)$ background represent $\boldsymbol{\Psi}_{2 \times 2}$. The regenerated $\boldsymbol{\Psi}_{4 \times 4}^{\prime}$ can be expressed in the following way:

$$
\boldsymbol{\Psi}_{4 \times 4}^{\prime}=\left(\begin{array}{cc|cc|}
\hline 0.5 & 0.6533 & 0.5 & 0.2706 \\
0.5 & 0.2706 & -0.5 & -0.6533 \\
\hline 0.5 & -0.2706 & -0.5 & 0.6533 \\
0.5 & -0.6533 & 0.5 & -0.2706 \\
\hline
\end{array}\right.
$$

where section highlighted with $(\square)$ background represents $\Psi_{1,1}^{\prime}$, ( $\square$ ) background 
represents $\Psi_{1,2}^{\prime},(\square)$ background represents $\Psi_{2,1}^{\prime}$ and ( $(-)$ background represents $\Psi_{2,2}^{\prime}$ respectively. Such representation is used in the proof of Theorem 3.5.1.

The block diagram depicting the modified Kronecker-based recovery using the newly generated sparsifying basis is shown in Figure 3.2.

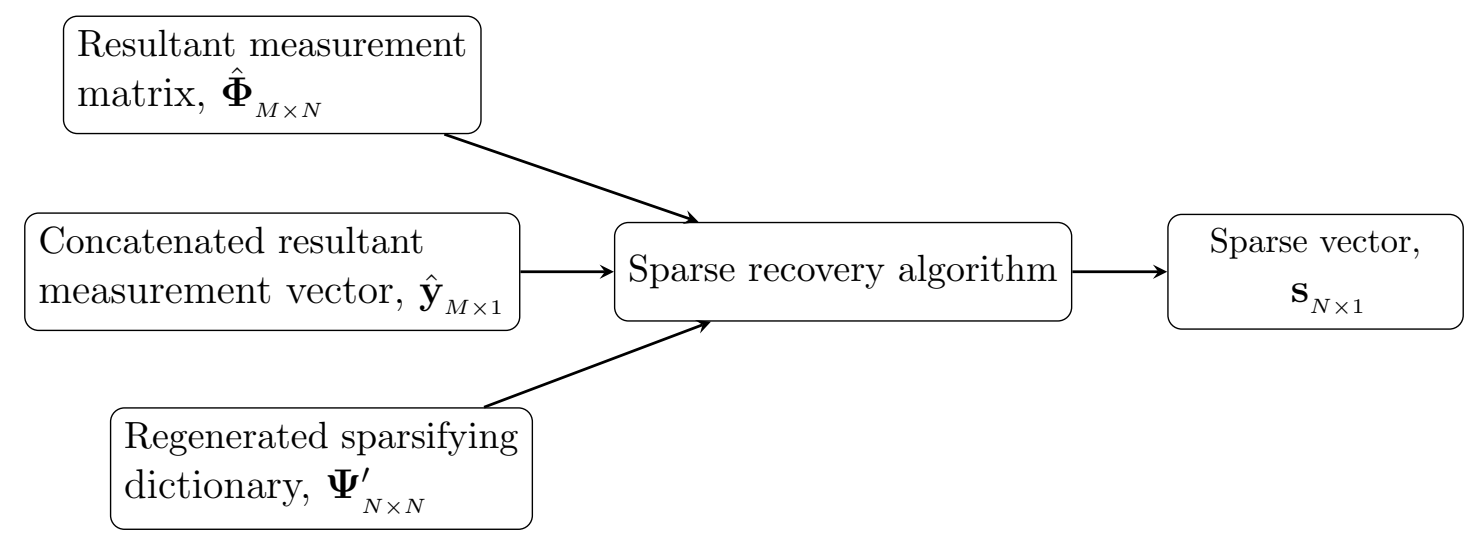

Figure 3.2: Block diagram representation of the modified Kronecker-based recovery technique.

This regeneration of a highly resolved sparsifying matrix lowers the mutual coherence between the resultant measurement matrix and sparsifying basis, when compared to the standard Kronecker-based technique, as shown by the theorem given below:

Theorem 3.5.1. Consider resultant Kronecker-based sparsifying matrix $\hat{\mathbf{\Psi}}_{N \times N}=$ $\mathbf{I}_{p \times p} \otimes \boldsymbol{\Psi}_{N^{\prime} \times N^{\prime}}$. If the modified sparsifying basis $\boldsymbol{\Psi}_{N \times N}^{\prime}$ is regenerated from the same orthonormal basis used in the standard Kronecker-based technique, then (also see [49]),

$$
\mu\left(\hat{\mathbf{\Phi}}_{M \times N}, \hat{\mathbf{\Psi}}_{N \times N}\right) \geqslant \mu\left(\hat{\mathbf{\Phi}}_{M \times N}, \mathbf{\Psi}_{N \times N}^{\prime}\right)
$$

Proof. The resultant measurement matrix, $\hat{\mathbf{\Phi}}$, is formed by Kronecker productbased expansion (refer to equation 3.7) in both the standard and the modified Kronecker-based recovery technique. Recalling the definition of mutual coherence 
(Chapter 2 - Section 2.5) $\mu(\boldsymbol{\Phi}, \mathbf{\Psi})=\max _{i \neq j}\left|<\tilde{\Phi}_{i}, \Psi_{j}>\right|{ }^{1}$, in order to prove that the decrease in $\mu$ in the modified Kronecker-based, it is suffice to show the following: $\max _{i \neq j}\left|<\tilde{\hat{\Phi}}_{i}, \hat{\Psi}_{j}>\right| \geqslant \max _{i \neq j}\left|<\tilde{\hat{\Phi}}_{i}, \Psi_{j}^{\prime}>\right|$.

Based on the definition of mutual coherence,

$$
\begin{array}{r}
\mu\left(\hat{\boldsymbol{\Phi}}_{M \times N}, \hat{\mathbf{\Psi}}_{N \times N}\right)=\max _{i \neq j}\left|<\tilde{\hat{\Phi}}_{i}, \hat{\Psi}_{j}>\right| \\
\mu\left(\hat{\boldsymbol{\Phi}}_{M \times N}, \boldsymbol{\Psi}_{N \times N}^{\prime}\right)=\max _{i \neq j}\left|<\tilde{\hat{\Phi}}_{i}, \Psi_{j}^{\prime}>\right|
\end{array}
$$

Mutual coherence between $\boldsymbol{\Phi}$ and $\boldsymbol{\Psi}$ can be concisely rewritten ${ }^{2}$ as $\mu(\boldsymbol{\Phi}, \boldsymbol{\Psi})=$ $\|\tilde{\Phi} \Psi\|_{\max }[48]$ where $\|\cdot\|_{\max }$ represents the max norm and is given by the largest entry of the matrix. Hence, $\mu$ for the standard and the modified Kronecker-based technique can be written in the following way:

$$
\begin{aligned}
\mu\left(\hat{\boldsymbol{\Phi}}_{M \times N}, \hat{\mathbf{\Psi}}_{N \times N}\right)= & \left\|\tilde{\boldsymbol{\Phi}}_{N^{\prime} \times M^{\prime}} \boldsymbol{\Psi}_{N^{\prime} \times N^{\prime}}\right\|_{\max } \\
\mu\left(\hat{\boldsymbol{\Phi}}_{M \times N}, \boldsymbol{\Psi}_{N \times N}^{\prime}\right)=\| & \left\|\begin{array}{cccc}
\tilde{\boldsymbol{\Phi}}_{N^{\prime} \times M^{\prime}} \boldsymbol{\Psi}_{1,1} & \tilde{\boldsymbol{\Phi}}_{N^{\prime} \times M^{\prime}} \boldsymbol{\Psi}_{1,2} & \ldots & \tilde{\boldsymbol{\Phi}}_{N^{\prime} \times M^{\prime}} \boldsymbol{\Psi}_{1, p} \\
\tilde{\boldsymbol{\Phi}}_{N^{\prime} \times M^{\prime}} \boldsymbol{\Psi}_{2,1} & \tilde{\boldsymbol{\Phi}}_{N^{\prime} \times M^{\prime}} \boldsymbol{\Psi}_{2,2} & \ldots & \tilde{\boldsymbol{\Phi}}_{N^{\prime} \times M^{\prime}} \boldsymbol{\Psi}_{2, p} \\
\vdots & \vdots & \vdots & \vdots \\
\tilde{\boldsymbol{\Phi}}_{N^{\prime} \times M^{\prime}} \boldsymbol{\Psi}_{p, 1} & \tilde{\boldsymbol{\Phi}}_{N^{\prime} \times M^{\prime}} \boldsymbol{\Psi}_{p, 2} & \ldots & \tilde{\boldsymbol{\Phi}}_{N^{\prime} \times M^{\prime}} \boldsymbol{\Psi}_{p, p}
\end{array}\right\|_{\max }
\end{aligned}
$$

Since, $\quad\left\|\tilde{\boldsymbol{\Phi}}_{N^{\prime} \times M^{\prime}} \boldsymbol{\Psi}_{l, k}\right\|_{\max } \leq\left\|\tilde{\boldsymbol{\Phi}}_{N^{\prime} \times M^{\prime}} \boldsymbol{\Psi}_{N^{\prime} \times N^{\prime}}\right\|_{\max } \forall k, l=1,2, \ldots, p$

Hence, $\mu\left(\hat{\boldsymbol{\Phi}}_{M \times N}, \hat{\mathbf{\Psi}}_{N \times N}\right) \geq \mu\left(\hat{\boldsymbol{\Phi}}_{M \times N}, \mathbf{\Psi}_{N \times N}^{\prime}\right)$.

It should be noted that equality will prevail when the standard Kronecker reconstruction technique is used. When equality prevails, no improvement should be

\footnotetext{
${ }^{1}$ Here $\tilde{\boldsymbol{\Phi}}=\boldsymbol{\Phi}^{T}$.

${ }^{2}$ The concise representation is not a standard matrix multiplication but an inner product operation between all possible columns of $\tilde{\boldsymbol{\Phi}}$ and $\boldsymbol{\Psi}$.
} 
expected in the recovery process when compared to regular CS recovery.

Because of the reduction in mutual coherence in the modified Kronecker-based CS technique, the quality of the recovered signals would improve as opposed to the standard Kronecker-based CS technique.

\subsection{Summary}

This chapter presents a mathematical formulation of Kronecker-based CS recovery. Kronecker-based measurement matrix expansion and the conditions to be maintained for successful recovery are also discussed. The standard and modified Kronecker-based recovery techniques are explained. Better recovery due to the reduction in mutual coherence between the Kronecker expanded measurement matrix and regenerated highly resolved sparsifying basis is also demonstrated mathematically. In the following chapters, applications of the modified Kronecker-based technique for recovery of 1-D and 2-D signals will be demonstrated. 


\section{Chapter 4}

\section{Kronecker-based Compressive Recovery for 1-D Signal ${ }^{1}$}

This chapter presents an application of the Kronecker-based CS recovery technique introduced in Chapter 2. Kronecker-based recovery technique on compressively measured 1-D bio-signal namely Electrocardiogram (ECG) is presented. Block-diagram of measurement framework, for both random and deterministic sensing, are given. Quality of improvement in reconstructed signal is analyzed using a number of metrics including signal-to-noise ratio (SNR), percentage root mean square difference (PRD) and correlation. Quality of the reconstructed ECG signals, acquired using deterministic measurement matrices are compared against that obtained using random matrices. Quality improvement in recovery while using Kronecker-based CS and its modified version and multiple sparsifying basis is also studied. A detailed statistical analysis for various compression ratios using ECG signals acquired from a total of 47 subjects from MIT-BIH Arrhythmia database [52] is presented. Noise analysis is conducted on an ECG signal, selected from 18 signals representing a wide 'variety of pathological

\footnotetext{
${ }^{1}$ Contents presented in this chapter have partially been published (or submitted for publication) in $[50,51]$ by the author.
} 
cases' to infer on the choice of measurement framework. Analysis reveals the superior performance offered by linear filtering-based deterministic measurement matrix over the random measurement matrices.

\subsection{Introduction}

The World Health Organization (WHO) has identified cardiovascular disease (CVD) as one of the leading causes of noncommunicable diseases (NCDs). According to 'World Health Statistics 2018', CVDs cause 44\% of deaths globally [53]. Due to an aging population in countries such as Canada, centralized health-care will no longer be an affordable solution. In order to avert a serious health care crisis, home-based health care monitoring is being considered. In addition, connected health and IoT devices are being accepted by users widely these days. Hence, continuous measurement of vital signs for monitoring purposes will soon become an essential part of a home-based health care monitoring system.

Recent developments in wireless communication and wearable sensor technology have enabled pervasive health monitoring which in turn has made continuous long term monitoring a reality. Continuous and remote ECG signals for monitoring purposes through Wireless Body Area Networks (WBAN) have the potential to offer almost real-time health care, health care anytime from anywhere, thus, lowering health care costs [54-60]. Such measurement solution for continuous monitoring should be cost-effective. To provide an affordable and low-cost solution, the monitoring devices are often battery powered, small scale (in size) with limited storage and computational capabilities. These wearable devices are capable of acquiring measurements and transmitting those measurements to a remote location for storage and further processing [61,62]. Kadrolkar et al. concluded that approximately $60 \%$ of energy 
of the battery-powered devices is consumed by wireless sensor nodes, responsible for data transmission [63]. As a result, frequent battery power replenishment or recharging is required to perform continuous monitoring. Hence long term and continuous ECG signal acquisition for monitoring become challenging. Alternatively, transmission operation could be done sparingly. Since measurements need to be stored and then transmitted either on demand or at some periodic intervals, reduction in the amount of data, i.e. compression, before transmission through the network may become necessary.

Recalling the formulation in Chapter 2, a compressible signal can be sensed and compressed at the same time using the CS-based measurement technique. As ECG signals are compressible, CS has been used as an effective technique for signal compression (and acquisition) [64-66]. In literature, a number of ECG compression algorithms have been proposed by earlier researchers [67-69]. By ensuring the simplicity in the encoder design for compression and minimizing the reconstruction error in the decoding process, CS-based ECG compression techniques have an edge over other techniques [70-72].

Following the discussions regarding the advantages of Kronecker-based CS recovery technique in Chapter 3, battery power of the small wearable devices would be drained in the generation of larger measurement matrices for continuous sensing in a resource-constrained environment. Hence, Kronecker-based expansion technique for ECG signal acquisition is an alternative to large measurement matrix generation (for compressive measurement). In this chapter, the modified Kronecker-based CS recovery technique would be applied to improve the quality of reconstruction of the compressively sensed ECG signals. 


\subsection{Segmentation-based Compressive ECG Mea- surement Techniques}

Traditionally, in the standard CS-based measurement approach, a large number of samples of the ECG signal would be compressively acquired for transmission. Instead, using a sensor capable of acquiring a smaller number of compressed measurements may be both cost effective and energy efficient. The measurements acquired by such sensors may be viewed as a segmented version of the original signal. The aforementioned technique to acquire a small number of compressed measurements can be viewed as segmentation-based sensing approach. In other words, one can view this as 'measuring independently'.

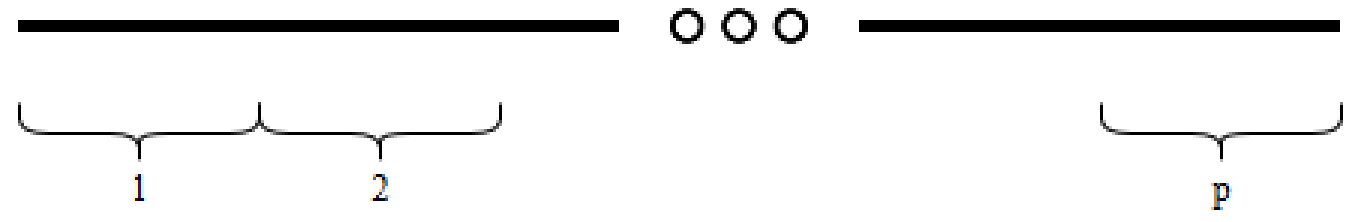

Figure 4.1: Signal segmentation into $p$ number of smaller blocks.

For example, in Figure 4.1, a sample ECG signal is segmented into $p$ smaller segments. Each segments can be considered as a separate measurement vector $\mathbf{y}$. This 'measuring independently' paradigm has the following advantages and disadvantages.

\subsubsection{Advantages}

1. With the reduction in measurement length, smaller measurement matrices can be constructed. Generation of measurement matrix in a smaller size would lead to lesser matrix multiplication. 
2. In order to perform continuous monitoring, ECG signals are transmitted wirelessly for further processing in a remote processor (which is assumed to have higher computational resources).

\subsubsection{Disadvantages}

Based on the discussion in Chapter 3, the standard Kronecker-based recovery of the ECG signal would lead to inferior reconstruction. Hence, a Kronecker-based technique is required to be explored to improve the signal quality during reconstruction, while performing the recovery only once.

\subsection{Way out?}

In order to address the aforementioned drawback, associated with inferior reconstruction, the modified Kronecker-based technique (discussed in Chapter 3 - Section 3.5) is used.

In order to perform continuous ECG acquisition using the principles of CS, an effective measurement matrix is required to be designed. Based on the classification of measurement matrices discussed in Chapter 2 - Section 2.4, segmented ECG signals can be acquired by either random measurement matrices or deterministic measurement matrices. In this section of the thesis, the performance of both random and deterministic measurement framework would be compared, in terms of quality of reconstruction and ease of design. 


\subsection{Random Measurement Framework}

Random measurement matrices, being one of most studied measurement matrices, are one of the natural candidates in designing compressive sensing framework for ECG signal acquisition. Figure 4.2 presents a framework for ECG signal acquisition using random measurement matrices.

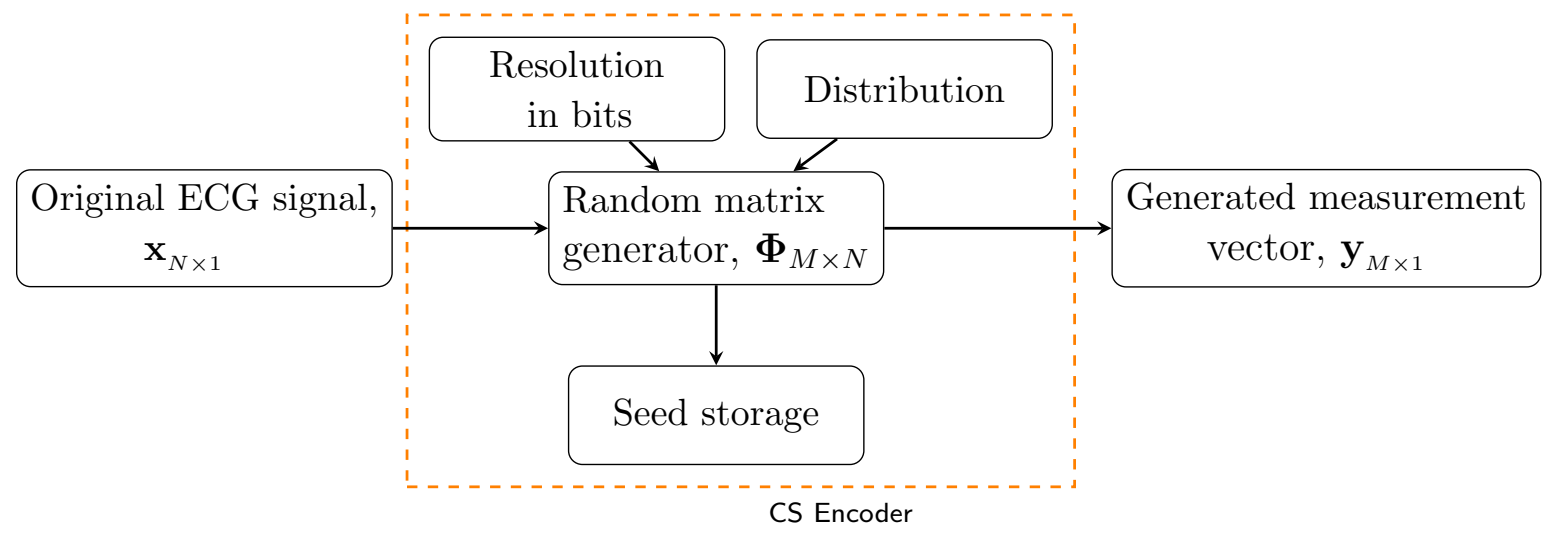

Figure 4.2: Block diagram representation of random measurement framework.

Above block diagram is generalised for designing random measurement matrices from a wide range of distribution. As a priori knowledge on the particular measurement matrix is required to be available during recovery, it is an effective solution to store the seed instead of storing the entire matrix for further realization. In this framework, the seed is transferred (or made available) to the remote processing unit along with the information of the specific distribution, from which the elements of the random matrix are drawn. With the help of the seed and the distribution, the same random matrix can be generated during recovery. In literature, random matrices with elements drawn from various distributions, such as Gaussian and sub-Gaussian Bernoulli distributions, have been used as measurement matrices to compressively acquire ECG [50,70,73-77]. A random number generator architecture was proposed based on inverse cumulative distribution function in Chung et al. [78]. This archi- 
tecture generated random matrices of different distributions, like Gaussian, for any given standard deviation $(\sigma)$. With such a framework using random measurement matrix, compressive measurements of ECG can be acquired.

\subsection{Discussions}

Random matrices used to measure ECG signal compressively in order to provide continuous and ambulatory monitoring come with the following advantages and disadvantages.

\subsubsection{Advantages}

1. Random matrices encode the acquired ECG signal, which cannot be deciphered without the a priori knowledge of the same matrix that was used in the measurement. Although the encryption provided by the random matrices is considered as 'very weak', transmission of the ECG data is relatively secure, as opposed to deterministic matrices, considering privacy of the individual. Although, modern crypto-systems provide better encryption to ensure privacy.

2. To optimize the signal quality, separate random matrices can be generated adaptively for $p$ individual segments. At the expense of computational complexity, oftentimes random matrices would provide the flexibility of adaptive measurement matrix construction.

\subsubsection{Disadvantages}

1. One of the biggest drawbacks of random measurement framework is the complexity of random matrix generation. As discussed in [35], hardware-based gen- 
eration of random matrix of size $M \times N$ would require $M \times N$ multiplication. Considering the measurement framework to be designed for resource constraint environments, random matrix generation would lead to faster draining of battery.

2. Based on the discussions in Chapter 2 - Section 2.4.1, random matrices provide guarantee in recovery with a probabilistic notion. Considering the need to monitor ECG continuously for patients in need, a probabilistic notion in recovery offered by random measurement framework might not be very practical to be considered. Since recovery is not always guaranteed, it becomes problematic to use random measurement framework for vital sign compression and recovery.

3. While using random measurement matrix framework, morphology of the ECG signal would not be preserved in the compressed domain. Applications where signal morphology is required to be preserved in the compressed domain, random measurement framework is not suitable.

To avoid these aforementioned disadvantages, deterministic measurement framework is being used as an alternative.

\subsection{Deterministic Measurement Framework}

Figure 4.3 represents the deterministic measurement framework for segmentationbased compressive ECG measurement. Recalling the classification of deterministic measurement matrices in Chapter 2 - Section 2.4.2, any of the deterministic matrix can be realised in the 'CS Encoder' block. However, the selection of a deterministic matrix depends on a few parameters, including ease of implementation in a small battery powered hardware and reconstruction quality of the recovered ECG signal. 


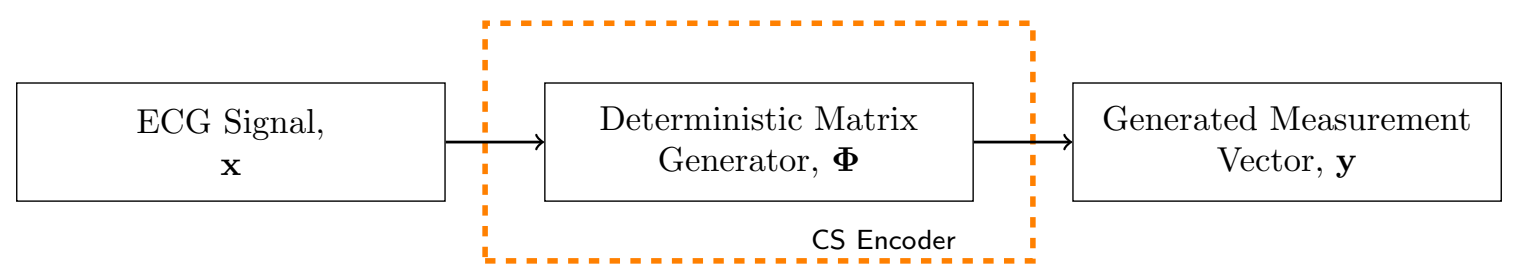

Figure 4.3: Block diagram representation of deterministic measurement framework.

This is to be noted that the construction of deterministic matrix can be performed during recovery, without the need to transfer the same matrix (or the seed) that was used during compressive measurement.

\subsection{Problem Formulation and Kronecker-based Recovery}

Let the ECG signal, $\mathbf{x}_{N \times 1}$ of length $N$ be segmented into $p$ smaller segments each of length $n$.

\subsubsection{Segmentation-based ECG Signal Representation}

The segmented signal can be represent in a matrix form, $\mathbf{X}_{n \times p}$ as shown below by equation 4.1,

$$
\mathbf{X}_{n \times p}=\left[\begin{array}{cccc}
\mathbf{x}_{1}^{1} & \mathbf{x}_{1}^{2} & \ldots & \mathbf{x}_{1}^{p} \\
\mathbf{x}_{2}^{1} & \mathbf{x}_{2}^{2} & \ldots & \mathbf{x}_{2}^{p} \\
\vdots & \vdots & \ldots & \vdots \\
\mathbf{x}_{n}^{1} & \mathbf{x}_{n}^{2} & \ldots & \mathbf{x}_{n}^{p}
\end{array}\right]
$$

The superscript of each column of $\mathbf{X}_{n \times p}$ represents individual segments (or segment number). For example, $\mathbf{x}^{1}$ represents the first segment. Similarly, other $p$ segments can be identified. The above matrix can be viewed as a collection of seg- 
ments of the original ECG measurement. A simplified form of equation 4.1 can be shown by the following equation 4.2 ,

$$
\mathbf{X}_{n \times p}=\left[\mathbf{x}_{j}^{i} \mid i=1,2, \ldots, p ; j=1,2, \ldots, n\right]
$$

Here, $i$ represents the number of segment, $j$ represents sample number and $n$ represents the length of each segment.

\subsubsection{Kronecker-based Measurement Matrix Formation for recovery}

For such segmentation-based approach, as the individual measurements of ECG signals can be represented in a consolidated matrix form, a measurement matrix is required to be formed based on the discussions on Kronecker-based measurement matrix expansion (please see Chapter 3 - Section 3.3.1 for expansion details). The resultant measurement matrix $\hat{\boldsymbol{\Phi}}$ can be represented by equation 4.3 , where Kronecker product is performed with the identity matrix of size $p \times p$ (or $\mathbf{I}_{p \times p}$ ) is performed.

$$
\hat{\mathbf{\Phi}}=\mathbf{I}_{p \times p} \otimes \boldsymbol{\Phi}_{m \times n}
$$

In context of $p$ segments of the ECG signal, a matrix form can be shown by equation 4.4, where $p$ diagonal elements (smaller measurement matrix for individual sections of size $m \times n)$ would form the resultant Kronecker-based measurement matrix $\hat{\boldsymbol{\Phi}}$. In equation 4.4, superscript of each $\boldsymbol{\Phi}_{m \times n}$ 's represents individual segments (or segment number). With the resultant measurement matrix, formed using the principles of Kronecker-based CS recovery, $p$ measurement vectors can be formed for $p$ individual segments. 


$$
\hat{\boldsymbol{\Phi}}=\left[\begin{array}{cccc}
\boldsymbol{\Phi}_{m \times n}^{1} & \mathbf{0} & \ldots & \mathbf{0} \\
\mathbf{0} & \boldsymbol{\Phi}_{m \times n}^{2} & \ldots & \mathbf{0} \\
& & \ddots & \\
\mathbf{0} & \mathbf{0} & \ldots & \boldsymbol{\Phi}_{m \times n}^{p}
\end{array}\right]
$$

where, $\mathbf{0}$ represents matrices of appropriate size having all elements equal to 0 .

\subsubsection{Segmented Measurement Vector Generation and Ma- trix Representation}

As discussed in the previous paragraph, $p$ number of smaller segments of length $n$ are measured by either of the above-mentioned measurement frameworks (random measurement framework or deterministic measurement framework discussed in Sections 4.4 and 4.6 respectively). For each smaller segment of length $n$, a measurement vector $\mathbf{y}_{m \times 1}$, where $m$ number of measurements is obtained in the compressed domain. Equation 4.5 represents the measurement vector generation for individual segments.

$$
\mathbf{y}_{m \times 1}=\boldsymbol{\Phi}_{m \times n} \mathbf{x}_{n \times 1}
$$

As a result, $p$ number of such measurement vectors would be generated, each of length $m$. These segmented measurement vectors can be represented in a matrix form shown below by equation 4.6 ,

$$
\mathbf{Y}_{m \times p}=\left[\begin{array}{cccc}
\mathbf{y}_{1}^{1} & \mathbf{y}_{1}^{2} & \ldots & \mathbf{y}_{1}^{p} \\
\mathbf{y}_{2}^{1} & \mathbf{y}_{2}^{2} & \ldots & \mathbf{y}_{2}^{p} \\
\vdots & \vdots & \ldots & \vdots \\
\mathbf{y}_{m}^{1} & \mathbf{y}_{m}^{2} & \ldots & \mathbf{y}_{m}^{p}
\end{array}\right]
$$


Here, each column represents individual compressed ECG measurements of length $m$. The compressed measurements can also be viewed as follows:

$$
\mathbf{Y}_{m \times p}=\left[\mathbf{y}_{j}^{i} \mid i=1,2, \ldots, p ; j=1,2, \ldots, m\right]
$$

In the above equation $4.7, i$ represents the number of segment whereas $j$ represents the sample number while $m$ is the length of individual segments.

In WBAN systems for continuous monitoring and ambulatory ECG monitoring, it is assumed that individual segments (i.e. columns of $\mathbf{Y}_{m \times p}$ matrix) are transmitted to the remote system for further processing. The remote processing unit is considered to have higher computational resources, and hence, is able to conduct the sparse recovery process (which is computationally expensive) of the compressed ECG measurements on demand.

\subsubsection{Kronecker-based Recovery of Segmented ECG Signals}

Once the segmented measurement vectors (acquired using $\hat{\mathbf{\Phi}}$ ) along with the Kronecker-based measurement matrix (preferably information of seed and distribution for the random measurement framework) are available, recovery can be performed by either the standard Kronecker-based recovery technique (discussed in Chapter 3 Section 3.4) or the modified Kronecker-based recovery technique (discussed in Chapter 3 - Section 3.5). Selection of sparsifying basis is an important when considering the quality of the reconstructed signal.

\subsubsection{Sparsifying Basis Selection}

Recovery of compressed ECG signals depend on the choice of sensing matrix and the choice of sparse basis for the representation of the measured signal. However, 
for measurement vector generation, only the measurement matrix need to be chosen. Once the selection of the measurement matrix is done, the same matrix should be used during the Kronecker-based recovery. Thus, the choice of a sparse basis will dictate the signal quality, once the measurement matrix has been chosen in the measurement or compression phase. In this thesis, the impact of using different bases $(\Psi)$ during the Kronecker-based recovery, under the two different measurement frameworks is investigated. Several options exist for the sparse basis selection that may be used for recovering compressed ECG signals from the segmented measurements. For instance, the choice may be an orthonormal basis, like wavelet and orthonormal non-wavelet basis such as discrete cosine transform (DCT) or discrete cosine transform-based transforms in time-frequency domain [79].

Basis selection for sparse recovery of ECG signals, from the compressed measurements, is a well-researched area. Wavelet-basis has been proposed to handle non-stationarities in the ECG signals. For instance, a tree-structure-based framework for ECG wavelet coefficients was proposed in [80]. Using the nesting property of wavelets, the concept of connected subtree has been proposed in [81]. However, there seems to be no study on the impact of choice of a basis on the recovery of compressed measurements.

In this thesis, an investigation on Kronecker-based recovery is conducted using a total of 77 different sparsifying representations using the following bases: DCT, Haar, discrete Meyer, 20 of Daubechies (Daubechies-1 to Daubechies-20), 15 of Biorthogonal (Biorthogonal-1.1 to Biorthogonal-6.8), 5 of Coiflets (Coiflets-1 to Coiflets-5), 15 of Reverse Biorthogonal (Reverse Biorthogonal-1.1 to Reverse Biorthogonal-6.8) and 19 of Symlets (Symlets-2 to Symlets-20) along with random measurement matrices (with Bernoulli and Gaussian distributions) and DBBD deterministic matrix frameworks.

Once the measurement vectors obtained by compressing $p$ smaller segments, using 
the measurement matrix and the sparsifying basis, sparse recovery algorithm can be applied to recover the original ECG signal $X_{N \times 1}$ from the compressed measurements.

In the next two sections, the standard and the modified Kronecker-based sparse recovery approach will be applied for compressed segmented ECG signal reconstruction.

\subsubsection{Standard CS Recovery for Segmented ECG Signal}

In segmentation-based ECG signal measurement, $p$ individual segments may be recovered individually to generate $p$ number of sparse vectors. In other words, with the $a$ priori knowledge of measurement matrix $\boldsymbol{\Phi}_{m \times n}$ and the sparsifying dictionary $\boldsymbol{\Psi}_{n \times n}$, sparse solution $\mathbf{s}_{n \times 1}$ is obtained using the measurement vector $\mathbf{y}_{m \times 1}$ (each column of $\mathbf{Y}_{m \times p}$ ). One of the aforementioned recovery algorithms (discussed in Chapter 2 - Section 2.5) is used for recovering the ECG signal from the compressed measurements. The general idea of recovery can be described as follows,

$$
\mathbf{s}_{n \times 1}=\Re\left(\mathbf{y}_{m \times 1}, \boldsymbol{\Phi}_{m \times n}, \boldsymbol{\Psi}_{n \times n}\right)
$$

where $\Re$ represents the recovery algorithm.

\subsubsection{Standard Kronecker-based Recovery for Segmented ECG Signal}

Instead of performing the computationally expensive recovery process $p$ times to obtain the sparse solution $\mathbf{s}_{n \times 1}$, a Kronecker-based solution is adopted (which is termed as the standard Kronecker-based approach in this thesis based on the discussions on the standard Kronecker-based recovery discussed in Chapter 3 - Section 3.4). Recalling the discussions on Kronecker-based CS recovery from Chapter 3.5, all columns of $\mathbf{Y}_{m \times p}$ (or measurements associated with individual segments of length $n$ ) are concate- 
nated to form a measurement vector $\mathbf{y}_{M \times 1}$ of length $M$ (where $M=p \times m$ ). Hence, with the availability of concatenated measurement vector $\mathbf{y}_{M \times 1}$, Kronecker-based resultant measurement matrix $\hat{\mathbf{\Phi}}_{M \times N}$ and Kronecker-based resultant sparsifying dictionary $\hat{\mathbf{\Psi}}_{N \times N}$; sparse vector $\mathbf{s}_{N \times 1}$ is recovered. The reconstruction problem can be formulated as below,

$$
\mathbf{s}_{N \times 1}=\Re\left(\mathbf{y}_{M \times 1}, \hat{\mathbf{\Phi}}_{M \times N}, \hat{\mathbf{\Psi}}_{N \times N}\right)
$$

where $\Re$ represents the recovery algorithm. Simplifying equation 4.9 with the total dictionary $\hat{\boldsymbol{\Theta}}_{M \times N}=\hat{\boldsymbol{\Phi}}_{M \times N} \hat{\boldsymbol{\Psi}}_{N \times N}$ following simplified form can be obtained,

$$
\mathbf{s}_{N \times 1}=\Re\left(\mathbf{Y}_{M \times 1}, \hat{\Theta}_{M \times N}\right)
$$

Formation of $\hat{\boldsymbol{\Theta}}_{M \times N}$ is given by 4.11 , as shown below,

$$
\hat{\boldsymbol{\Theta}}_{M \times N}=\mathbf{I}_{p \times p} \otimes \hat{\boldsymbol{\Theta}}_{m \times n}=\mathbf{I}_{p \times p} \otimes\left(\boldsymbol{\Phi}_{m \times n} \boldsymbol{\Psi}_{n \times n}\right)
$$

A matrix-based representation of $\hat{\boldsymbol{\Theta}}_{M \times N}$ can be expressed by equation 4.12. The representation follows:

$$
\hat{\Theta}_{M \times N}=\left[\begin{array}{cccc}
\Phi_{\mathbf{m} \times \mathbf{n}} \boldsymbol{\Psi}_{\mathbf{n} \times \mathbf{n}} & 0 & \ldots & 0 \\
0 & \boldsymbol{\Phi}_{\mathbf{m} \times \mathbf{n}} \boldsymbol{\Psi}_{\mathbf{n} \times \mathbf{n}} & \ldots & 0 \\
\vdots & \vdots & \ldots & \vdots \\
0 & 0 & \ldots & \Phi_{\mathbf{m} \times \mathbf{n}} \Psi_{\mathbf{n} \times \mathbf{n}}
\end{array}\right]
$$

where, $\mathbf{0}$ represents matrices of size $m \times n$ having all elements equal to 0 .

Recalling the preservation of $\mu$ (discussed in Chapter 3 - Section 3.4), quality of the recovered signals obtained by using the standard Kronecker-based recovery 
technique would not be any different from that of the individual recovery of $p$ smaller segments. So, the quality degradation caused by the segmentation of the ECG signal cannot be dealt with using the standard Kronecker-based recovery technique. In the next section, the modified Kronecker-based recovery technique is discussed in order to improve the quality of the recovered ECG signals.

\subsubsection{Modified Kronecker-based Recovery for Segmented ECG Signal}

In order to perform the modified Kronecker-based recovery of the segmented ECG signals, similar approach of forming $\mathbf{y}_{M \times 1}$ (concatenating $p$ individual segments of size $m$ ) and Kronecker-based resultant measurement matrix $\hat{\boldsymbol{\Phi}}_{M \times N}$ is followed (also see the previous Section 4.7.4.3). To obtain the sparse vector $\mathbf{s}_{N \times 1}$ using equation 4.9 , the information of the sparsifying basis is needed. Let us denote the resultant sparsifying basis in the modified Kronecker-based recovery technique as $\hat{\Psi}^{\prime}$. From the discussions on the construction of sparsifying basis in Chapter 3 - Section 3.5, $\hat{\mathbf{\Psi}}_{N \times N}^{\prime}$ is a newly formed matrix from the same basis that is being considered in the standard Kronecker-based recovery.

Hence, in order to obtain the sparse vector $\mathbf{s}_{N \times 1}$, a modified form of equation 4.9 need to be used. The modified formulation is as follows:

$$
\mathbf{s}_{N \times 1}=\Re\left(\mathbf{y}_{M \times 1}, \hat{\mathbf{\Phi}}_{M \times N}, \hat{\mathbf{\Psi}}_{N \times N}^{\prime}\right)
$$

A simplified version of equation 4.10 can be represented by the following equation 4.14 ,

$$
\mathbf{s}_{N \times 1}=\Re\left(\mathbf{Y}_{M \times 1}, \hat{\Theta}_{M \times N}^{\prime}\right)
$$

where $\hat{\mathbf{\Theta}}_{M \times N}^{\prime}=\hat{\boldsymbol{\Phi}}_{M \times N} \hat{\mathbf{\Psi}}_{N \times N}^{\prime}$ signifies the total dictionary for the modified Kronecker- 
based recovery technique. A Kronecker product for obtaining the total dictionary is shown below,

$$
\hat{\boldsymbol{\Theta}}_{M \times N}^{\prime}=\left(\mathbf{I}_{p \times p} \otimes \boldsymbol{\Phi}_{m \times n}\right) \boldsymbol{\Psi}_{N \times N}^{\prime}
$$

A matrix form of representing equation 4.15 is shown below,

$$
\hat{\boldsymbol{\Theta}}_{M \times N}^{\prime}=\left[\begin{array}{cccc}
\boldsymbol{\Phi}_{m \times n} & \mathbf{0} & \ldots & \mathbf{0} \\
\mathbf{0} & \boldsymbol{\Phi}_{m \times n} & \ldots & \mathbf{0} \\
\vdots & \vdots & \ldots & \vdots \\
\mathbf{0} & \mathbf{0} & \ldots & \boldsymbol{\Phi}_{m \times n}
\end{array}\right] \boldsymbol{\Psi}_{N \times N}^{\prime}
$$

In the above equation, $\mathbf{0}$ signifies matrices of size $m \times n$ with elements equal to 0 .

Based on the discussions in the previous chapter, $\mu$ is decreased while using the modified Kronecker-based recovery technique. Hence, an improvement in the quality of the reconstructed ECG signals is observed. Results and analysis presented in the next sections validates this claim.

\subsection{Performance Evaluation Metrics}

To analyze the performance of the modified Kronecker-based recovery technique, the following metrics are used. In this section, $\mathbf{x}$ has been considered as the original signal of length $N, \hat{\mathbf{x}}$ is the recovered signal and $\|\cdot\|_{2}$ represents the $\ell_{2}$ norm. 


\subsubsection{Compression Ratio (CR)}

$\mathrm{CR}$ is defined in the following way,

$$
C R=\left(1-\frac{M}{N}\right) \times 100 \%
$$

\subsubsection{Percentage Root Mean square Difference (PRD)}

PRD is defined as follows:

$$
P R D=\frac{\|\mathbf{x}-\hat{\mathbf{x}}\|_{2}}{\|\mathbf{x}\|_{2}} \times 100 \%
$$

\subsubsection{Normalized Percentage Root Mean square Difference (PRDN)}

Normalized version of PRD has been considered by earlier researchers to make the measurement independent of DC value of $\mathbf{x}[82]$.

$$
\operatorname{PRDN}=\frac{\|\mathbf{x}-\hat{\mathbf{x}}\|_{2}}{\|\mathbf{x}-\overline{\mathbf{x}}\|_{2}} \times 100 \%
$$

Here, $\overline{\mathbf{x}}$ signifies the mean of the original signal $\mathbf{x}$.

Another approach to quantify the quality of the reconstructed signal, $\hat{\mathbf{x}}$, is to represent $\mathrm{PRDN}$ in $\mathrm{dB}$ form. This representation of PRDN is termed signal-to-noise ratio (SNR) by past researchers and is defined as follows:

$$
S N R=20 \log _{10}\left(\frac{\|\mathbf{x}-\overline{\mathbf{x}}\|_{2}}{\|\mathbf{x}-\hat{\mathbf{x}}\|_{2}}\right)
$$


The above equation can also be written as,

$$
S N R=-20 \log _{10}(0.01 \times P R D N)
$$

\subsubsection{Root Mean Square (RMS) Error}

In [83], RMS has been used for comparing $\mathbf{x}$ and $\hat{\mathbf{x}}$ based on error measurement. It is defined as follows:

$$
R M S=\frac{\|\mathbf{x}-\hat{\mathbf{x}}\|_{2}}{\sqrt{\mathbf{N}}}
$$

where $\mathrm{N}$ is the length of the signal.

\subsubsection{Zero-th lag of Cross-Correlation (CC)}

The zero-th lag of cross-correlation between $\mathbf{x}$ and $\hat{\mathbf{x}}$ provides the quality metric.

$$
\phi_{\mathbf{x}, \hat{\mathbf{x}}}(0)=\sum_{i=0}^{N-1}[\mathbf{x}(i) \hat{\mathbf{x}}(i)]
$$

Note that this metric is used to compare the same length of reconstruction.

\subsubsection{Quality Score (QS)}

Fira et al. introduced a metric to assess the quality of reconstructed signal $\hat{\mathbf{x}}$ by combining compression and the measure of reconstruction error of the signal [84]. QS is defined as a ratio of CR and PRD,

$$
Q S=\frac{C R}{P R D}
$$

For a given CR, higher the QS, better the quality of the recovered signal. 


\subsubsection{Maximum Amplitude Error (MAX)}

Maximum amplitude error measures the signal distortion of the reconstructed signal $\hat{\mathbf{x}}$ and defined as follows [85],

$$
M A X=\max |\mathbf{x}-\hat{\mathbf{x}}|
$$

Low MAX value signifies lesser distortion present in the recovered signal $\hat{\mathbf{x}}$, i.e. better recovery. This measure can be used for a given CR.

\subsection{Database Description}

In order to analyze the performance, ECG signals from the MIT-BIH Arrhythmia database [52] are considered. This two-channel database of ECG signals contains a record a total of 47 subjects comprised of 25 men and 22 women, representing different age groups. Digitization of the records were performed at a rate of 360 samples/second per channel over a range of $10 \mathrm{mV}$ with 11-bit resolution [86,87]. MIT-BIH Arrhythmia database is often considered as a 'benchmark database' for ECG signals. As a result, in this thesis MIT-BIH Arrhythmia database is chosen to perform analysis.

\subsection{Results and Analysis}

For this thesis, the first 512 samples, i.e. the length of original ECG signal $N=512$, from each signal is chosen. The ECG signal is then segmented into $p=8$ segments. Individual segments are of length $n=\frac{512}{8}=64$. While using random sensing matrices, results are averaged over 500 realizations. 
In the following sections of this chapter, the impact of various levels of wavelet decomposition, change in $\mathrm{CR}$, the impact of measurement noise on the quality of the recovered ECG signal are discussed. Statistical analysis is presented as part of the evaluation.

\subsubsection{Impact of Wavelet Decomposition Levels}

Figure 4.4 and Figure 4.5 show the comparison between the increase in SNRs, by comparing the SNRs obtained by performing the modified Kronecker-based recovery technique to that of the standard Kronecker-based recovery technique, at 10 different decomposition levels for two distinct wavelet basis, Symlets-8 and Daubechies-15. DBBD matrix of size $32 \times 64$ has been used as measurement matrix $(\boldsymbol{\Phi})$ for $\mathrm{CR}$ $=50 \%$. However, for different decomposition levels, the variation in SNR increase is negligible for these two wavelets. Hence, wavelets with lower decomposition levels can be used as a sparsifying basis, as opposed to that of the higher decomposition levels, without appreciable change in SNR.

Figure 4.6 shows the SNR obtained by averaging over all 47 signals present in the MIT-BIH Arrhythmia database, while using the modified Kronecker-based recovery technique with $\boldsymbol{\Phi}=\mathrm{DBBD}$ at $\mathrm{CR}=50 \%$. Wavelet bases at different decomposition levels are used as sparsifying bases. Figure 4.7 represents the similar results for the recovered signal obtained using the standard Kronecker-based recovery technique. Results clearly show that the SNRs of the recovered signals do not change considerably, while changing the decomposition level of the wavelets. There is a significant improvement in the SNRs when recovery is done using the modified Kronecker technique. For both the analyses, recovery is performed using all of the aforementioned wavelets, as sparsifying bases, and the best wavelet from each wavelet family has been chosen for presentation in this thesis. For example, Biorthogonal-3.7 wavelet 


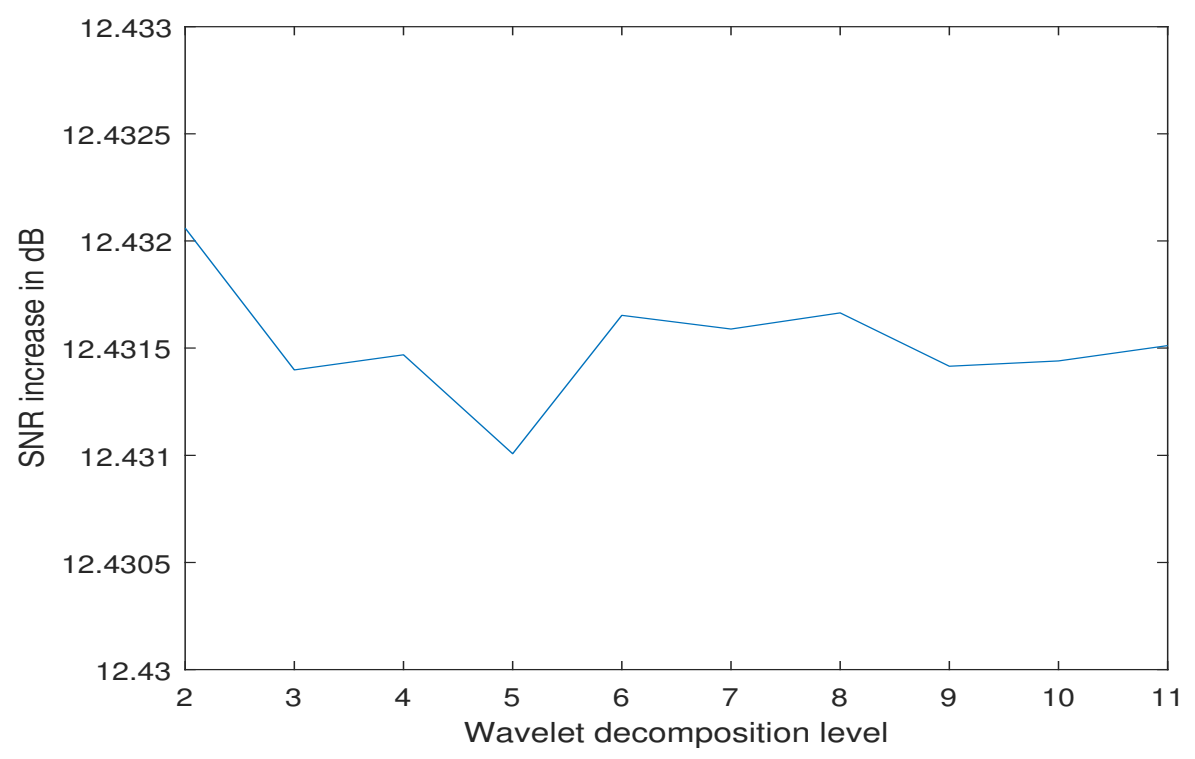

Figure 4.4: Comparison between different decomposition levels of Daubechies-15 wavelet at $\mathrm{CR}=50 \%$ with $\boldsymbol{\Phi}=\mathrm{DBBD}$.

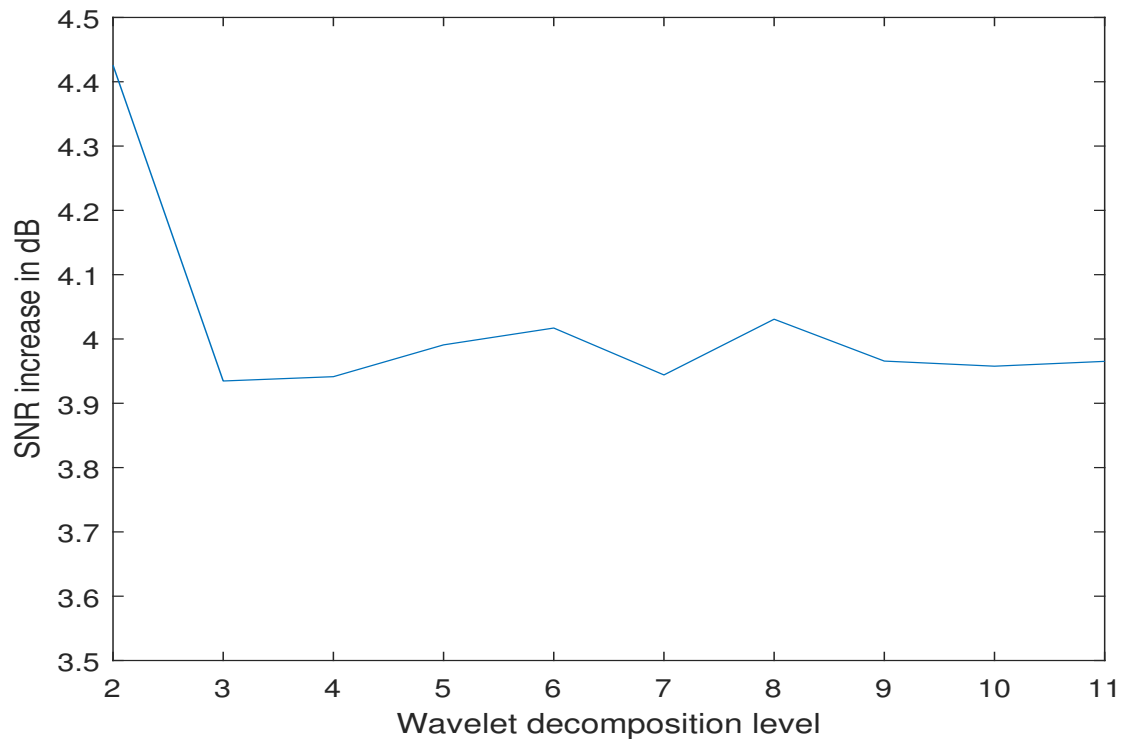

Figure 4.5: Comparison between different decomposition levels of Symlets- 8 wavelet at $\mathrm{CR}=50 \%$ with $\boldsymbol{\Phi}=\mathrm{DBBD}$.

outperformed other wavelets from the Biorthogonal wavelet family.

Results in Table 4.1 presents a statistical analysis performed on all 47 ECG signals 


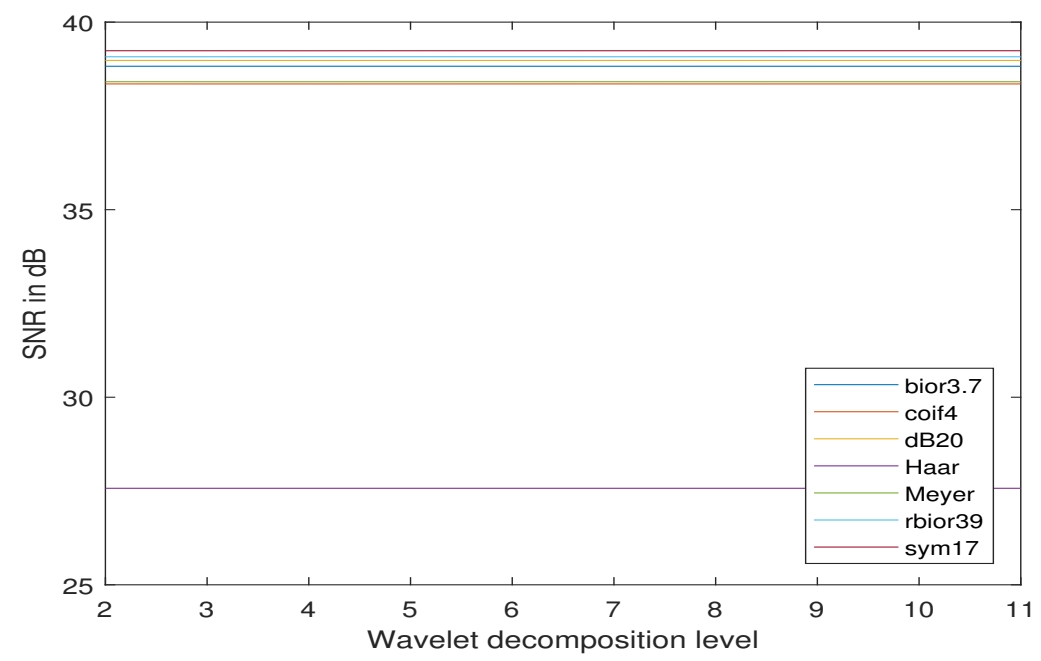

Figure 4.6: Comparison between different decomposition levels of different wavelets at $\mathrm{CR}=50 \%$ with $\boldsymbol{\Phi}=\mathrm{DBBD}$ using the modified Kronecker-based recovery technique.

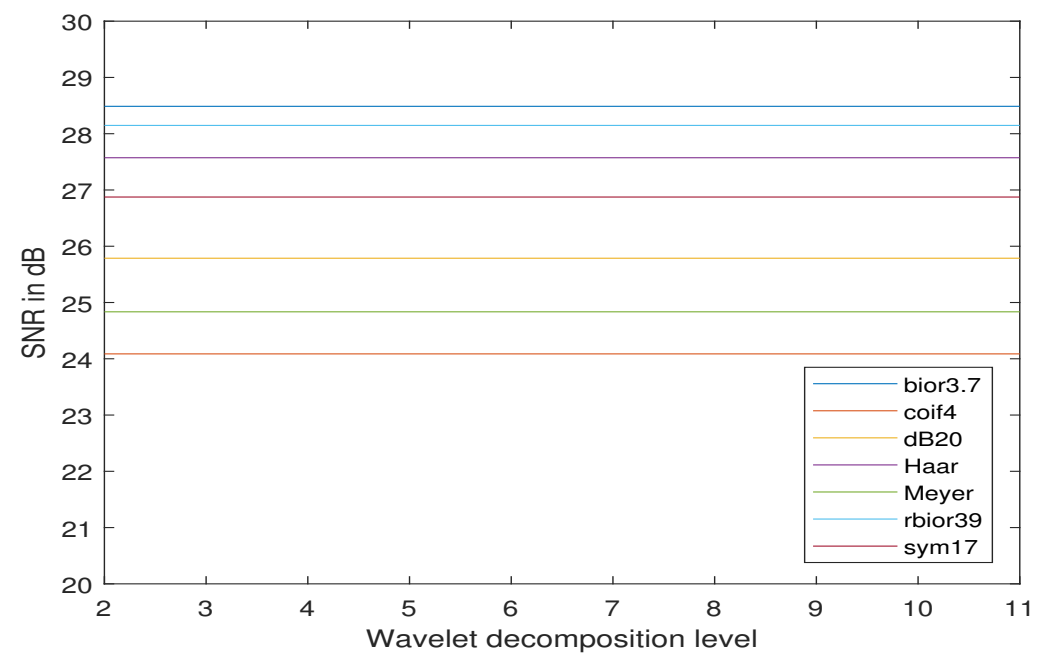

Figure 4.7: Comparison between different decomposition levels of different wavelets at $\mathrm{CR}=50 \%$ with $\boldsymbol{\Phi}=\mathrm{DBBD}$ using the standard Kronecker-based recovery technique. 
Table 4.1: Statistical analysis of recovery performance using the modified Kroneckerbased technique: $\mathrm{CR}=50 \%, \boldsymbol{\Phi}=\mathrm{DBBD}$

\begin{tabular}{|ccccccccc|}
\hline Statistical Parameter & \multicolumn{10}{c|}{ SNR $(\mathrm{dB})$} \\
\hline & Biorthogonal & Coiflets & Daubechies & DCT & Haar & Discrete Meyer & Reverse Biorthogonal & Symlets \\
Minimum & 5.31 & 21.69 & 17.45 & 35.17 & 20.79 & 31.19 & 12.46 & 17.45 \\
\hline Maximum & 26.61 & 23.65 & 26.76 & 35.17 & 20.79 & 31.19 & 27.00 & 36.62 \\
\hline Median & 21.96 & 22.90 & 23.20 & 35.17 & 20.79 & 31.19 & 23.08 \\
\hline
\end{tabular}

available in the database. SNRs are obtained using the modified Kronecker-based technique with 77 wavelet dictionaries as sparsifying basis $(\boldsymbol{\Psi})$ for all 47 ECG signals. Average SNR over all 47 signals for each basis is calculated. Then average SNRs are calculated for each family of the wavelet. For example, when Daubechies (Daubechies1 to Daubechies-20) wavelet family is used as $\boldsymbol{\Psi}$, in the first step average (averaged over 47 signals) SNRs are calculated for each of the 20 wavelets in the Daubechies family. In the second step, average (averaged over all 20 wavelets of Daubechies family) SNR was obtained for the Daubechies wavelet family. The process is repeated for all 7 other wavelet families. The minimum, maximum and median of the recovered SNR for each sparsifying basis are also obtained and presented. Results indicate that DCT outperforms other wavelet-based dictionaries when DBBD sensing matrix is used for measurement along with the modified Kronecker-based recovery technique. From Table 4.1 it is be inferred that the variation in signal quality of reconstruction can be observed while using Biorthogonal, Daubechies, Reverse Biorthogonal and Symlets. Whereas similar wide variation cannot be seen for Coiflets basis. This is to be noted that DCT, Haar and Discrete Meyer basis do not constitute families. Hence, no variation in signal quality is observed. As $\mu$ does not depend on CR, for higher CRs the similar trend would be observed. Although the averaged SNRs would decrease with an increase in CR. 


\subsubsection{Impact of CR}

In Figure 4.8, the qualitative comparison of the recovered signals by using the modified Kronecker-based recovery technique to that of the standard Kronecker-based recovery technique is presented. DBBD is used as measurement matrix with Biorthogonal-3.3 wavelet as sparsifying basis. For this analysis, level 2 decomposition is chosen. The performance has been analyzed by two of the aforementioned performance evaluation metrics, SNR and quality score. Results obtained for three different CRs, $50 \%, 75 \%$ and $87.5 \%$, indicate that the modified Kronecker-based recovery technique outperforms its standard counterpart for all CRs. Figure 4.9 and Figure 4.10 show the performance analysis using the random matrix in the measurement framework, instead of using DBBD deterministic matrix framework. In Figure 4.9 performance analysis is done for the elements of random $\boldsymbol{\Phi}$ drawn from the normal or Gaussian distribution whereas for Figure 4.10 Bernoulli distribution is chosen. In [50], by comparing the performance of both the recovery techniques on a range of ECG signals, it is concluded that the random matrix with Bernoulli distribution performs better, marginally than that of the normal distribution with DCT as a sparsifying basis while recovery. However, the difference is nominal for Biorthogonal-3.3 wavelet as a sparsifying basis. Whereas comparing the performance analysis for both DBBD and random matrices as $\boldsymbol{\Phi}$ for the same sparsifying basis, Biorthogonal-3.3 wavelet at level 2 decomposition, DBBD deterministic matrix outperforms the random matrices as $\boldsymbol{\Phi}$ for all CRs. It is to be noted that for higher CRs, like $75 \%$, better performance can be obtained using the deterministic sensing matrix. To analyze this, consider

the results shown in Figure 4.8 and Figure 4.9. It can be seen from Figure 4.8, with $\mathrm{CR}=75 \%$, SNR obtained by the modified Kronecker-based recovery technique for $\boldsymbol{\Phi}$ $=$ DBBD is $20.16 \mathrm{~dB}$. Whereas from Figure 4.9, for $\boldsymbol{\Phi}$ being a random matrix with 
normal distribution records SNR of $4.59 \mathrm{~dB}$, under the identical conditions. A 15.57 $\mathrm{dB}$ improvement in SNR of the recovered signal is obtained by using the DBBD deterministic matrix as a measurement matrix $(\boldsymbol{\Phi})$, as opposed to using random matrices for the same for $\mathrm{CR}=75 \%$.
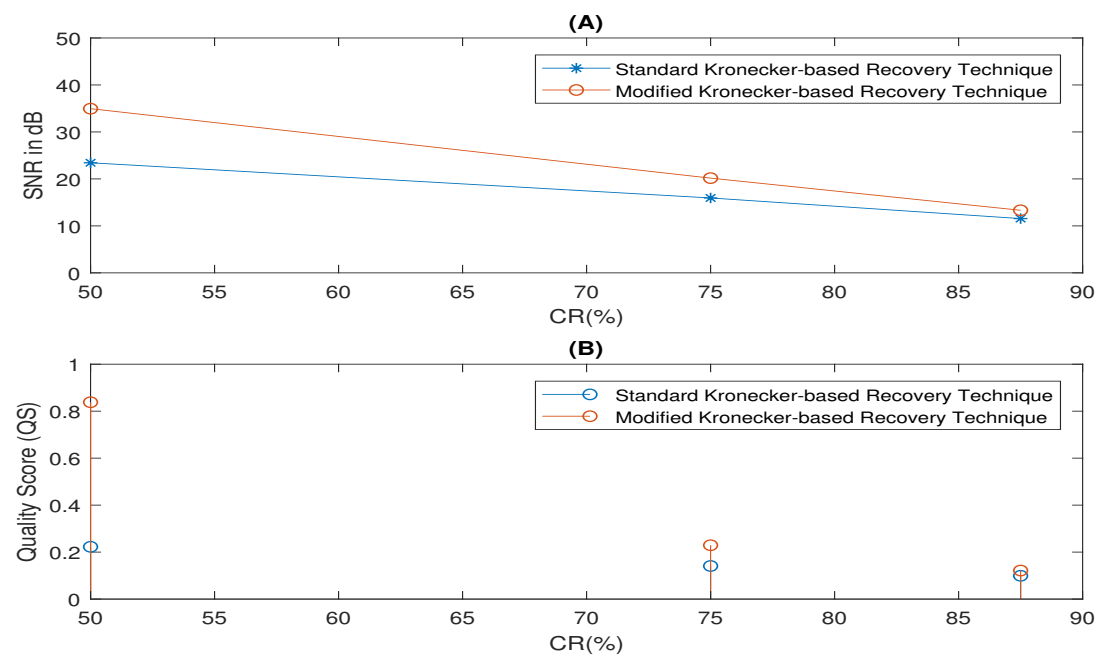

Figure 4.8: Reconstruction quality comparison on for various levels of compression using $\boldsymbol{\Psi}=$ Biorthogonal-3.3 wavelet basis with $\boldsymbol{\Phi}=$ DBBD. (A) Comparison of SNR as a function of CR. (B) Comparison of Quality Score (QS) as a function of CR.

\subsubsection{Analysis of Quality of Reconstruction of Compressed ECG Signals}

From Table 4.2 a quantitative comparison, based on the RMS error, Quality Score (QS) and maximum amplitude error (MAX), is presented for three different measurement matrices (random matrices with elements drawn from Gaussian and Bernoulli distribution and DBBD deterministic matrix) with DCT sparsifying basis for $\mathrm{CR}=$ $50 \%$. The result clearly indicates that the modified Kronecker-based technique results in a higher quality score (QS) in comparison to the standard Kronecker-based technique. This also indicates the quality improvement of the recovered ECG signal. 

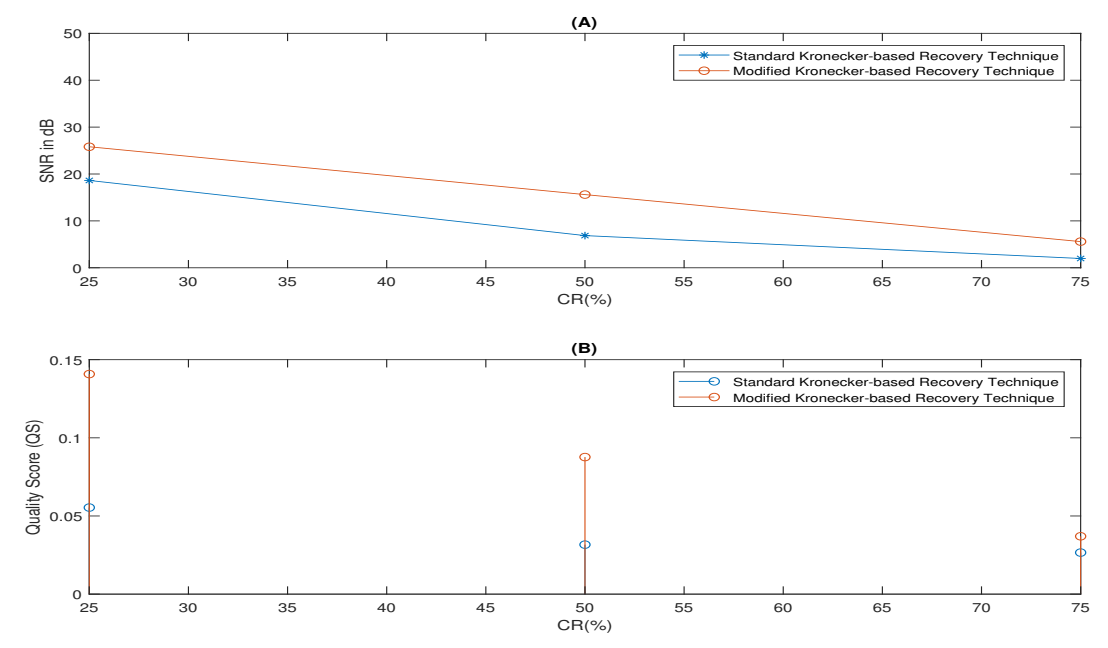

Figure 4.9: Reconstruction quality comparison on for various levels of compression using $\boldsymbol{\Psi}=$ Biorthogonal-3.3 wavelet basis and $\boldsymbol{\Phi}$ as a random matrix with values drawn from normal distribution. (A) Comparison of SNR as a function of CR. (B) Comparison of Quality Score (QS) as a function of CR.
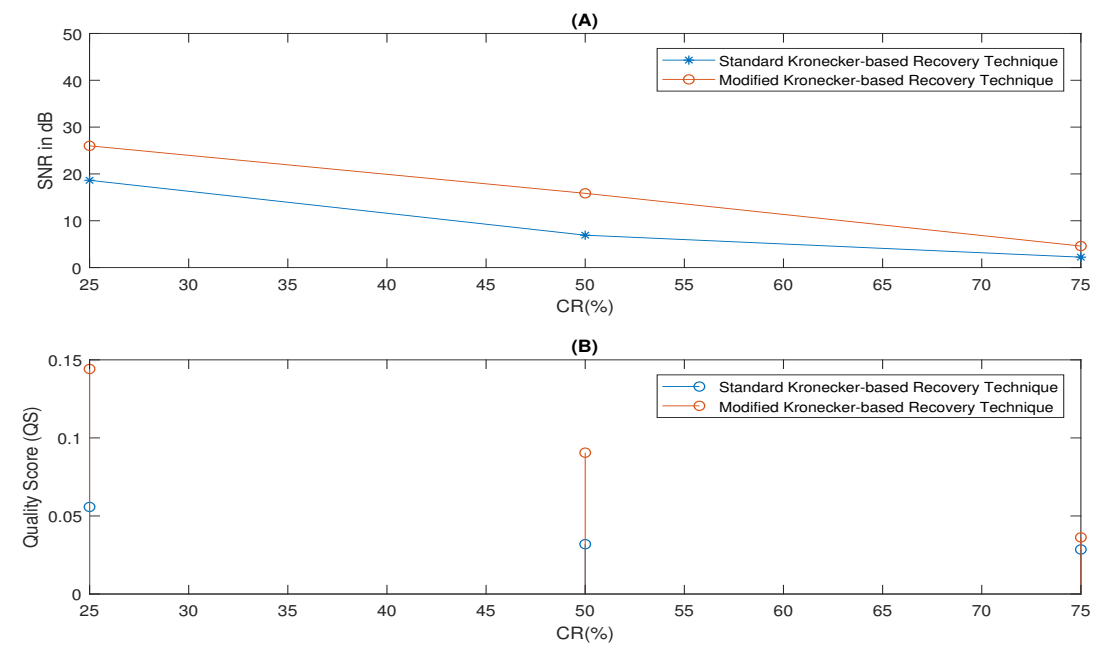

Figure 4.10: Reconstruction quality comparison on for various levels of compression using $\boldsymbol{\Psi}=$ Biorthogonal-3.3 wavelet basis and $\boldsymbol{\Phi}$ as a random matrix with values drawn from Bernoulli distribution. (A) Comparison of SNR as a function of CR. (B) Comparison of Quality Score (QS) as a function of CR. 
Table 4.2: Analysis of reconstruction quality using the standard and the modified Kronecker-based technique for $\mathrm{CR}=50 \%$

\begin{tabular}{|c|c|c|c|c|c|c|c|c|c|c|}
\hline \multirow{2}{*}{ Signal Number* } & \multirow{2}{*}{$\Phi$} & \multirow{2}{*}{$\Psi$} & \multicolumn{4}{|c|}{ Standard Kronecker-based Technique } & \multicolumn{4}{|c|}{ Modified Kronecker-based Technique } \\
\hline & & & SNR & RMS Error & QS & MAX & SNR & RMS Error & QS & MAX \\
\hline 115 & DBBD & DCT & 38.48 & 1.33 & 0.43 & 0.07 & 38.58 & 0.94 & 0.61 & 0.05 \\
\hline 115 & $\begin{array}{lr}\text { Random Matrix } & \text { Gith Gaussian } \\
\text { with } & \text { Gistribution }\end{array}$ & $\mathrm{DCT}$ & 14.17 & 18.73 & 0.03 & 0.73 & 19.63 & 10.50 & 0.06 & 0.71 \\
\hline 115 & $\begin{array}{lr}\text { Random } & \text { Matrix } \\
\text { with } & \text { Bernoulli } \\
\text { distribution }\end{array}$ & DCT & 15.88 & 16.07 & 0.04 & 0.62 & 20.33 & 9.44 & 0.06 & 0.55 \\
\hline
\end{tabular}

*Following signal is one of the 18 signals available in the MIT-BIH Arrhythmia database identified by medical practitioners as a representative of a pathological case $[82]$.

The visual quality improvement further shows that better quality of the recovered ECG signal may be achieved by using deterministic sensing matrix, such as DBBD, instead of using random matrices. The inferences made here do not depend on CR.

Figure 4.11 shows the recovery of the original ECG signal (signal number 115 of the MIT-BIH Arrhythmia database) using the modified Kronecker-based technique with $\boldsymbol{\Phi}=$ DBBD and DCT as a sparsifying basis. ECG signal 115 from the MIT-BIH Arrhythmia database is chosen. According to [82], signal number 115 represents a pathologically important signal. From 4.1 it is shown that DCT sparsifying basis provides better signal quality. 1536 samples from the beginning of the available ECG signal has been considered, i.e. $N=1536$. Now, $N$ samples are segmented into $p=24$ segments. Hence, each smaller segment is having a length of $n=64$. Besides visual realization of the recovery, analysis result also confirms very low recovery error, therefore ensuring the quality of the recovered ECG signal using the modified Kronecker-based technique. 

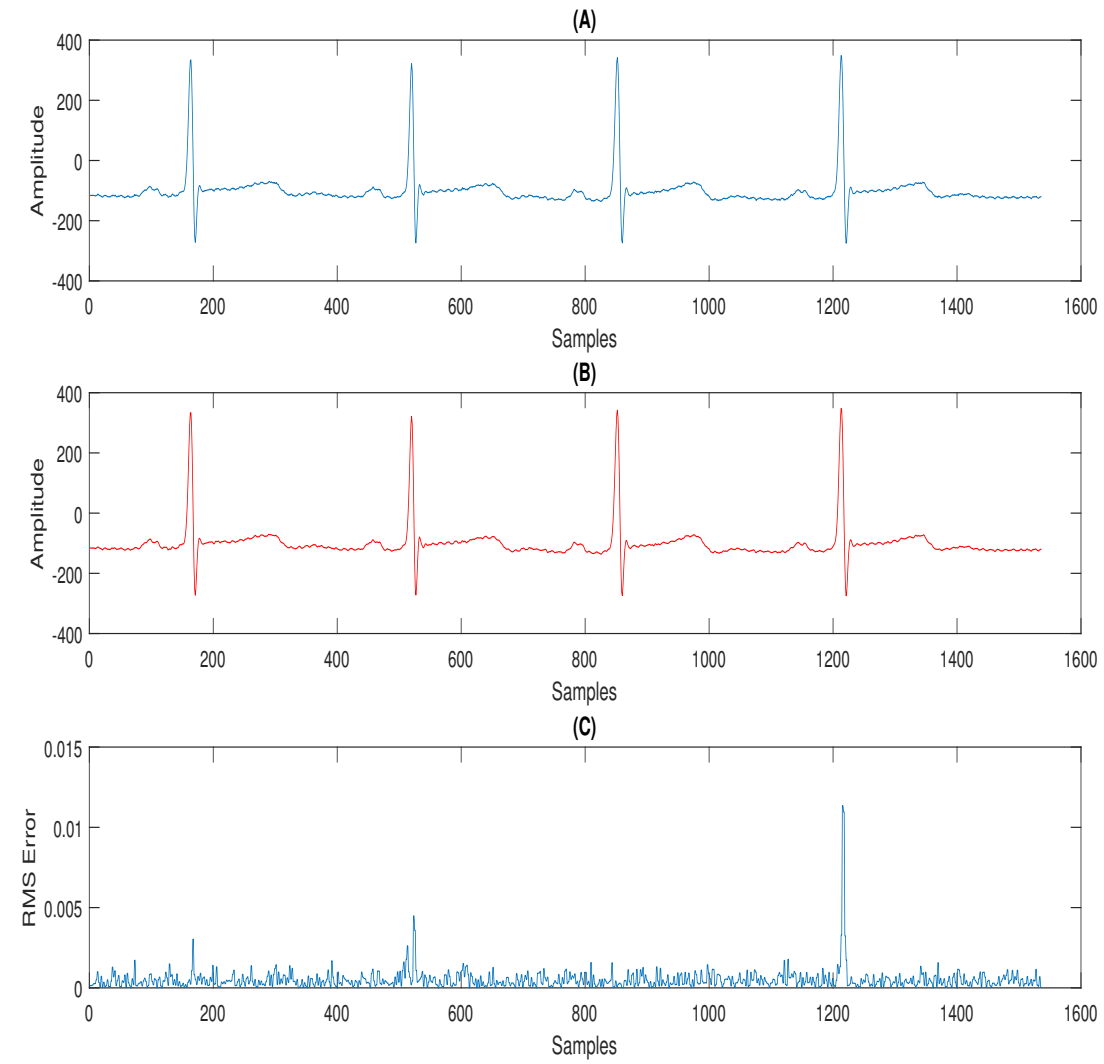

Figure 4.11: Visual representation of ECG signals. Recovery has been performed using SL0 algorithm, $\boldsymbol{\Phi}=$ DBBD and $\mathrm{CR}=50 \%$ with $\boldsymbol{\Psi}=$ DCT. (A) Original signal (x). (B) Recovered signal ( $\hat{\mathbf{x}})$. (C) Reconstructed signal quality assessment based on RMS error. 


\subsubsection{Recovery of Noisy ECG: Choice of Measurement Framework}

In order to demonstrate the advantage of using DBBD matrix in a noisy environment, the ECG signal (115 signal considered above) is then corrupted by $-15 \mathrm{~dB}$ additive Gaussian noise. The first 512 points of this corrupted signal are considered for compression. Since both random and deterministic frameworks are to be considered, the sparsifying matrix that is favourable for the random matrix is chosen. As random matrices with elements drawn from the Bernoulli distribution outperforms that of the normal distribution, the Bernoulli random matrix is chosen for the analysis. Coiflets-5 basis is identified as the best basis among all 77 basis that are considered in this thesis. Therefore, Coiflets-5 basis is used as $\boldsymbol{\Psi}$. When compressed with DBBD matrix, the same $\boldsymbol{\Psi}$ is used. This sparsifying basis is not the best basis for DBBD matrix (Refer to 4.2 ). $\mathrm{CR}=75 \%$ is chosen as the recovered signal quality degrades for higher CRs. Note that with $\mathrm{CR}=87.5 \%$, recovered ECG signal cannot be visually identified from measurements obtained using a random matrix.

To assess the visual quality of the recovered ECG signals obtained in presence of noise, reconstruction error is obtained by finding the difference between the original ECG signal $(\mathbf{x})$ to that of the reconstructed ECG signal $(\hat{\mathbf{x}})$, recovered from a noisy measurement. Figure 4.12, using DBBD as $\boldsymbol{\Phi}$, and Figure 4.13, using random matrix as $\boldsymbol{\Phi}$ with elements drawn from Bernoulli distribution, represent the visual recovery of the measurement for $\mathrm{CR}=75 \%$ while $-15 \mathrm{~dB}$ Gaussian noise is added (resembling measurement noise). It is evident from Figure 4.12, that the recovered signal $\hat{\mathbf{x}}$ can clearly be distinguished as an ECG signal by using DBBD matrix whereas the morphology of $\hat{\mathbf{x}}$ is not maintained by using random Bernoulli measurement matrix framework, shown in Figure 4.13. The reconstruction error obtained while using 
DBBD matrix is considerably lower than that of the $\boldsymbol{\Phi}_{\text {Bernoulli }}$, quantifying the robustness of the deterministic measurement framework in the presence of additive Gaussian noise in ECG.
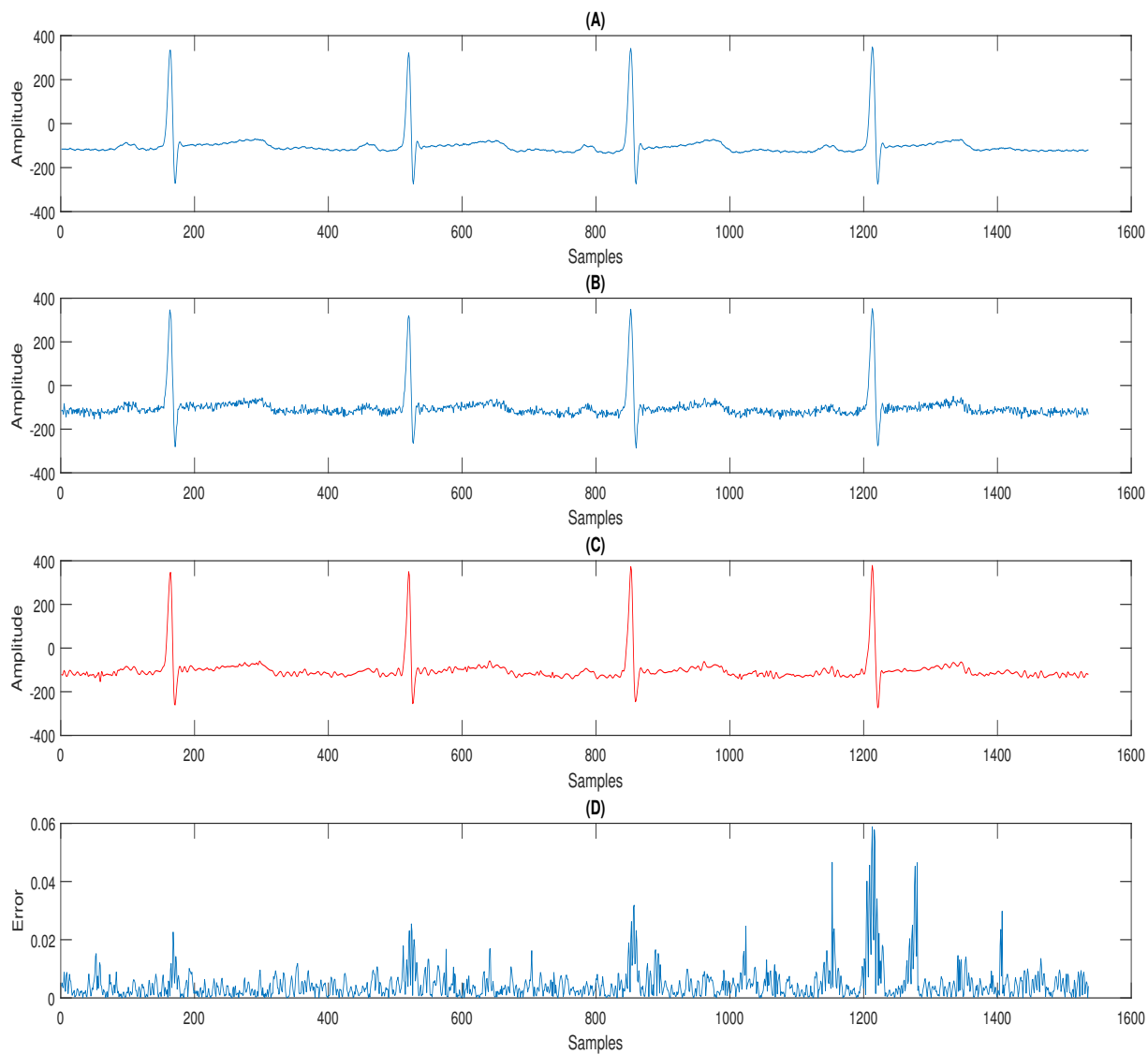

Figure 4.12: Visual representation of ECG signals in presence of -15 dB noise at source (before compression). Recovery has been performed using SL0 algorithm, $\boldsymbol{\Phi}=\mathrm{DBBD}$ and $\mathrm{CR}=75 \%$ with $\boldsymbol{\Psi}=$ Coiflets-5. (A) Original signal (x). (B) Signal corrupted by $-15 \mathrm{~dB}$ additive noise $\left(\mathbf{x}^{\prime}\right)$. (C) Recovered signal ( $\left.\hat{\mathbf{x}}\right)$. (D) Reconstructed signal quality assessment based on RMS Error. 

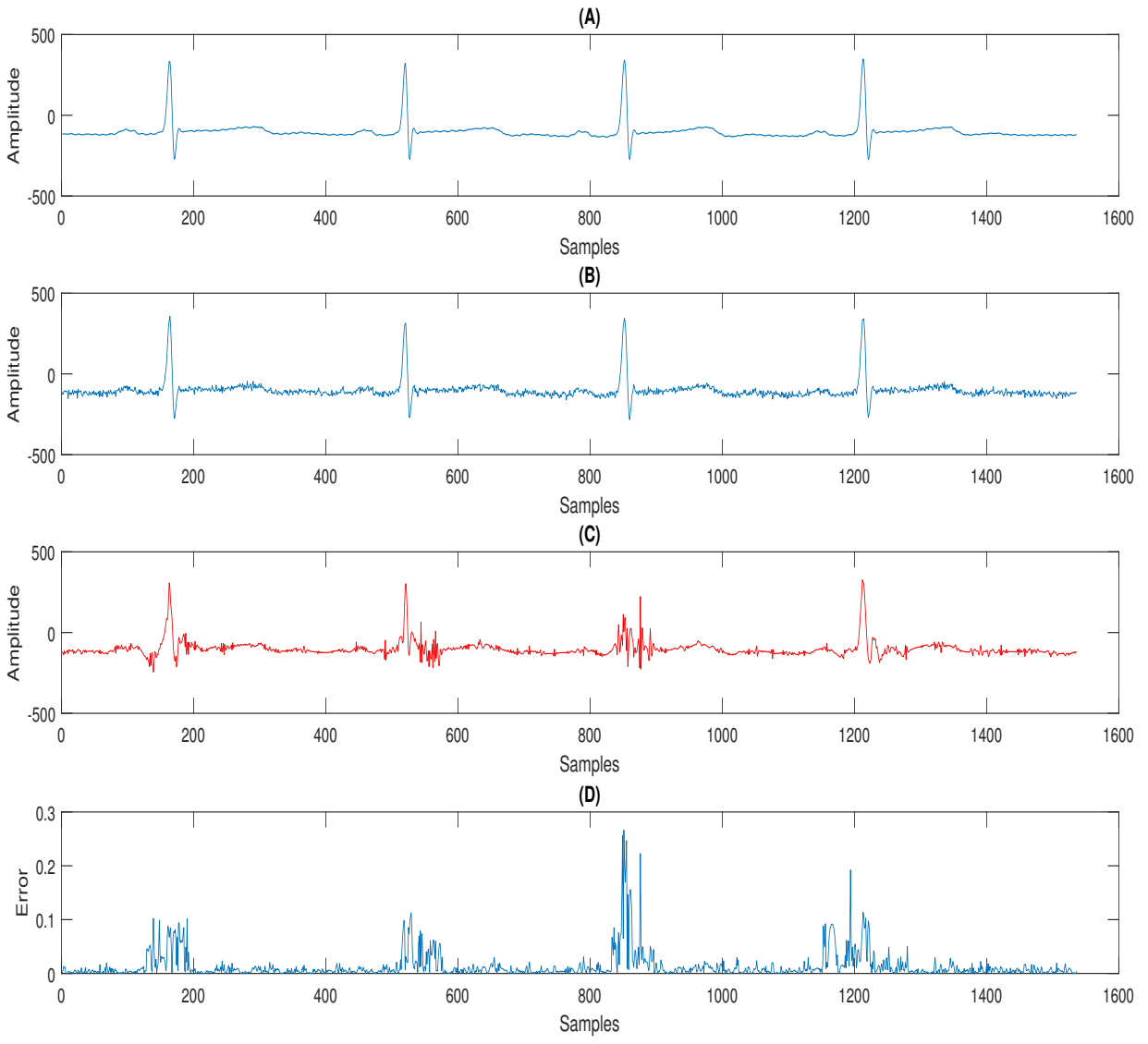

Figure 4.13: Visual representation of ECG signals in presence of $-15 \mathrm{~dB}$ noise at source (before compression). Recovery has been performed using SL0 algorithm, $\mathbf{\Phi}=$ Random matrix with values chosen from Bernoulli distribution and $\mathrm{CR}=75 \%$ with $\Psi=$ Coiflets-5. (A) Original signal (x). (B) Signal corrupted by $-15 \mathrm{~dB}$ additive noise $\left(\mathbf{x}^{\prime}\right)$. (C) Recovered signal $(\hat{\mathbf{x}})$. (D) Reconstructed signal quality assessment based on RMS Error. 


\subsection{Summary}

In this chapter, an application of the modified Kronecker-based sparse reconstruction technique, in 1-D signals, is presented. A problem statement related to continuous ECG monitoring (segmentation-based), using Kronecker-based CS recovery framework is introduced. Random and deterministic measurement framework for compression and recovery is presented with block diagrams. Advantages and disadvantages of both the frameworks and potential areas of applications are also discussed. A matrix-based formulation of the segmentation-based ECG measurement is shown in this chapter. The segmentation-based approach leads to the degradation of quality in the recovered signal. Using the modified Kronecker-based technique, the quality of the recovered signal is improved. A total of 47 ECG signals from the MIT-BIH Arrhythmia database are used to perform analysis in order to validate the improvement in reconstructed signal quality using the modified Kronecker-based CS recovery technique. 7 metrics are used to quantify quality improvement in recovery. Various choices of sparsifying basis for a given measurement matrix is also taken into account. While using the wavelet family as a basis, it is observed that recovery using lower level decomposition provided the same quality recovered signals when using higher levels of decomposition. A statistical analysis on the quality of reconstruction using the modified Kronecker-based technique is also presented. Impact of $\mathrm{CR}$ on the signal quality has been presented for both random and deterministic CS-based measurement framework. Visual representation of the ECG signal recovery has been plotted with 'RMS error' as a metric to show improvement in quality, while using the modified Kronecker-based recovery technique. Quality of the signal reconstruction in presence of noise is also assessed. Linear filter-based DBBD measurement matrix has outperformed random matrix-based measurement in presence of noise at source. This 
work shows that the deterministic matrix may be better suited for ECG signals, if no encryption is required during signal acquisition.

In the next chapter, the Kronecker-based CS recovery technique is extended to 2-D signals. 


\section{Chapter 5}

\section{Kronecker-based Compressive Recovery for 2-D Signal ${ }^{1}$}

In this chapter of the thesis, the application of Kronecker-based CS recovery technique in improving the quality of compressively sensed 2-D signals, such as images is studied. Two different measurement techniques: column-wise measurement (or row-wise, depending on which is compressed, sometimes also referred in this thesis as row/column-wise) and a modified 2-D measurement (sometimes also referred as row \& column-wise) CS techniques are presented. In this work, the Kronecker-based CS recovery technique is expanded to the 2-D signals. A comparative analysis is presented for these two measurement techniques after the recovery is accomplished using the standard and the modified Kronecker-based techniques. Magnetic Resonance (MR) images chosen from NCIGT [89] database is used to demonstrate the improvement in signal quality (obtained by using Kronecker-based CS recovery technique). A statistical analysis based on structural similarity and reconstruction error is also presented, for varying CRs.

\footnotetext{
${ }^{1}$ Contents presented in this chapter have partially been published (or submitted for publication) in [88] by the author.
} 


\subsection{Introduction}

One of the major problems with CS for 2-D signals is the size of the measurement matrix. Generation and storage of a large measurement matrix are challenging and often impractical. In CS for 2-D signals, a block-based approach is used. In this approach, the signal is first divided into small blocks. Then each block is then classified as either sparse or dense and CS is attempted only on the sparse blocks [90-92]. In [93], block Hadamard ensemble approach is used to make the sensing faster.

Although the block-based CS approach significantly reduces the size of the sensing matrix, all the smaller blocks are required to be reconstructed as a whole. As a result, reconstruction of the signal becomes computationally complex. To solve this problem, a CS framework was developed for 2-D signals [2]. In this framework, sensing is done in a column-by-column or row-by-row fashion, using the same sensing matrix. In [94], the conditions for similarity between row-by-row and column-by-column measurement was explored. Exploiting the property of group sparsity, multiple measurement vector (MMV) model was introduced in [95]. In this model, a collection of 1-D signals was treated as a group of vectors (hence a matrix) and measured in column-wise approach while sparsification was achieved using a single common dictionary for all the vectors in the group. In the literature, few reconstruction algorithms have been proposed for the CS framework [94-96]. Although reconstruction is assumed to be done where computational power is not an issue, on the measurement or sensing side it would be advantageous to have small sensing matrices. This may be particularly important as the sensing devices are becoming low powered, with limited on-board storage.

To apply the theory of CS for 2-D signals, a larger sized $\boldsymbol{\Phi}$ would be needed. For example, an image $\mathbf{X}_{N \times N}$ would require measurement of $N \times N$ pixels. When the value of $N$ is large, generation of $\boldsymbol{\Phi}$ would be computationally demanding with 
a large storage requirement. To solve this issue, in CS individual column vectors are measured, i.e. $\mathbf{x}_{N \times 1}^{i}$ where $i=1,2 \ldots N$ and compression are carried out on an individual column as though a 1-D signal is being measured. In this scheme, a measurement matrix would require to be generated for individual columns, i.e. $\boldsymbol{\Phi}_{M \times N}^{i}$ where $i=1,2 \ldots N$. By taking $M$ linear measurements from $\mathbf{X}_{N \times N}$, in the aforementioned column-by-column fashion, resultant measurement matrix (formed by the measurement vectors) $\mathbf{Y}_{M \times N}$ can be formed [88].

\subsection{Column-wise 2-D CS}

Figure 5.1 presents the block diagram of the column-wise CS measurement and recovery of 2-D signals. Let us consider a 2-D signal $\mathbf{X}_{N \times N}$. In the column-wise CS measurement approach, $N$ individual columns of the matrix (each of length $N$ ) is considered as an 1-D signal of length $N$, i.e. $\mathbf{x}_{N \times 1}$. Recalling the generation of compressed measurements, stated in Equation 2.2 in Chapter 2, $N$ measurement vectors can be obtained in the following form:

$$
\mathbf{y}_{M \times 1}=\boldsymbol{\Phi}_{M \times N} \mathbf{x}_{N \times 1}
$$

Once the measurement vectors are obtained, sparse reconstruction is performed, using Equation 2.8, to obtain 1-D recovered signal, $\hat{\mathbf{x}}_{N \times 1}$. Repeating the process $N$ times (for $N$ individual columns), the recovered 2-D signal $\hat{\mathbf{X}}_{N \times N}$ is obtained.

\subsection{Segmentation-based CS}

To further reduce the size of the measurement matrix, each column may be segmented into smaller lengths and these smaller length columns may then be compressed indi- 


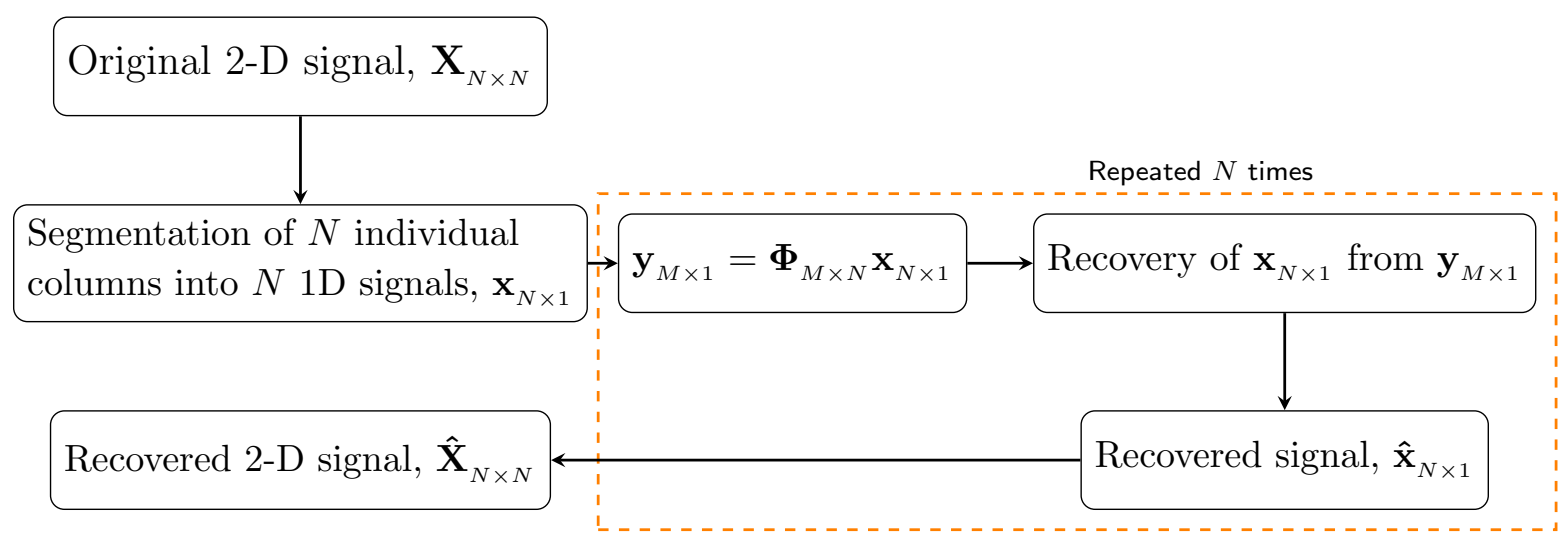

Figure 5.1: Column-wise measurement and recovery of 2-D signals.

vidually. This is termed as segmentation-based $C S$ in this thesis. In this work, the size of the measurement matrix is smaller than that of the column-wise approach of CS framework. From Figure 5.2, it can be seen that the size of measurement matrix $\boldsymbol{\Phi}$ would be $M \times N$, to measure (in a column-wise fashion) an image $\mathbf{X}_{N \times N}$. Whereas, once each columns are segmented into $p$ smaller segments, each of length $n$ (i.e. $N=n \times p$ ), size of the measurement matrix reduces to $m \times n$. As $n<N$, hence from Figure 5.3 it is evident that the size of the measurement matrix reduces. Hence, the generation of smaller measurement matrix suffice.

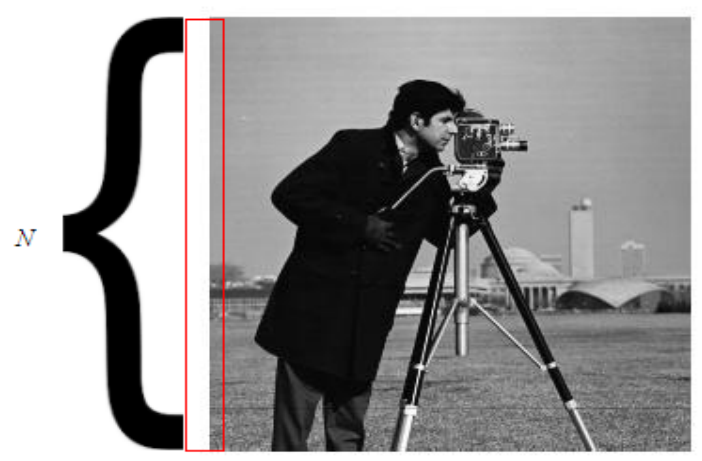

Figure 5.2: Non-segmented image with each column of length $N$.

Quality of the reconstructed signal from these individually compressed smaller 


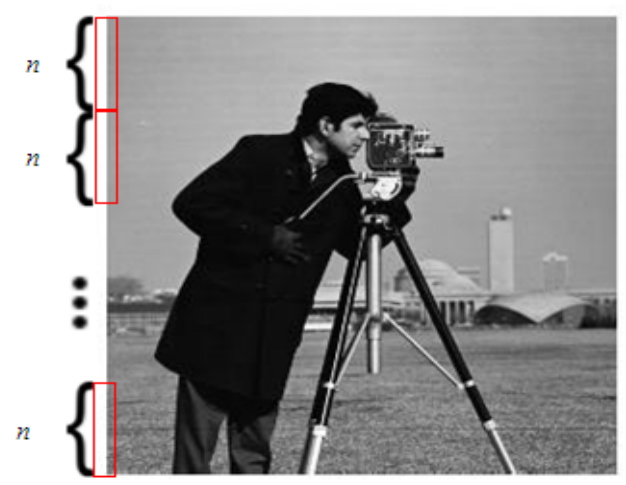

Figure 5.3: Column-wise segmented image with each segment of length $n$.

length columns is compromised (please refer to Chapter 4 - Section 4.2.2). To improve the quality of the reconstructed signal, the modified Kronecker-based CS technique is need to be applied. Images used in both Figure 5.2 and Figure 5.3 are made freely available by Image Processing Toolbox ${ }^{\mathrm{TM}}$ of MathWorks ${ }^{\circledR}$ [97].

\subsubsection{Segmentation-based 2-D Measurement}

In light of the discussions in Section 5.3, let us suppose that each column of the original image $\mathbf{X}_{N \times N}$ is segmented into $p$ smaller sections each of length $n(n<N)$. In other words, $p$ smaller 1-D signals of size $n$, i.e. $\mathbf{x}_{N \times 1}$, are formed. As a result, measurement matrices of size $m \times n$, i.e. $\boldsymbol{\Phi}_{m \times n}$ are required to be formed. As $n<N$, size of $\boldsymbol{\Phi}$ reduces significantly. Applications concerning random measurement matrices, where generation and storage of larger measurement matrices are not practical, segmentation-based approach is effective. As discussed in the previous section, $p$ measurement vectors $\mathbf{y}_{m \times 1}$ are formed (concatenating which entire column would be obtained). Recalling the discussion in Chapter 3 - Section 3.3.1, Kronecker-based CS approach is suitable for such measurement matrix expansion. Finally, $p$ number of $\hat{\mathbf{x}}_{N \times 1}$ are recovered and the reconstructed image $\hat{\mathbf{X}}_{N \times N}$ is obtained by concatenating 
them. Deterministic measurement matrix framework (discussed in Chapter 4 - Section 4.6) is used with DBBD matrix as $\boldsymbol{\Phi}$ [35]. DBBD matrix is easy to implement and will help to obtain superior quality of reconstructed images in comparison to the random measurement matrices.

In the following sections, results and analysis are presented by comparing the modified Kronecker-based CS recovery approach and its standard counterpart (also refer to Sections $\{3.4,3.5\}$ from Chapter 3 and Sections $\{4.4,4.6,4.7 .4 .3,4.7 .4 .4\}$ from Chapter 4 for the details of measurement matrix construction, the standard and the modified Kronecker-based CS recovery for segmentation-based 1-D signals).

\subsection{Performance Evaluation Metrics}

\subsubsection{Signal-to-Noise Ratio}

To quantify the improvement in reconstruction quality, signal-to-noise ratio (SNR) has been considered and is defined as follows:

$$
S N R(d B)=-20 \log _{10} \frac{\|(\mathbf{x}-\hat{\mathbf{x}})\|_{2}}{\|\mathbf{x}\|_{2}}
$$

where $\mathbf{x}$ and $\hat{\mathbf{x}}$ represent the original and reconstructed images. $\|\cdot\|_{2}$ represents the $\ell_{2}$ norm.

\subsubsection{Structural Similarity Index}

Structural similarity between the original and the reconstructed images have been compared by evaluating the Structural Similarity (SSIM) index, proposed by Wang et al. in [98]. Similarity between two images is quantified as a function of background 
luminance, contrast and the structure of both the images and expressed as follows,

$$
S S I M(\mathbf{x}, \hat{\mathbf{x}})=f(L(\mathbf{x}, \hat{\mathbf{x}}), C(\mathbf{x}, \hat{\mathbf{x}}), S(\mathbf{x}, \hat{\mathbf{x}})))
$$

where, $L$ and $C$ are functions comparing luminance and contrast between two images, the original image $\mathbf{x}$ and the recovered image $\hat{\mathbf{x}}$ and $S$ is the function to compare the structure of both the images, $\mathbf{x}$ and $\hat{\mathbf{x}}$ after variance normalization. In practice equation 5.3 is expressed in the following form:

$$
S S I M(\mathbf{x}, \hat{\mathbf{x}})=L(\mathbf{x}, \hat{\mathbf{x}})^{\alpha} \times C(\mathbf{x}, \hat{\mathbf{x}})^{\beta} \times S(\mathbf{x}, \hat{\mathbf{x}})^{\gamma}
$$

where $L(\mathbf{x}, \hat{\mathbf{x}})=\frac{2 \mu_{\mathbf{x}} \mu_{\hat{\mathbf{x}}}+A_{1}}{\mu_{\mathbf{x}}^{2}+\mu_{\hat{\mathbf{x}}}^{2}+A_{1}}, C(\mathbf{x}, \hat{\mathbf{x}})=\frac{2 \sigma_{\mathbf{x}} \sigma_{\hat{\mathbf{x}}}+A_{2}}{\sigma_{\mathbf{x}}^{2}+\sigma_{\hat{\mathbf{x}}}^{2}+A_{2}}$ and $S(\mathbf{x}, \hat{\mathbf{x}})=\frac{\sigma_{\mathbf{x} \hat{\mathbf{x}}}+A_{3}}{\sigma_{\mathbf{x}} \sigma_{\hat{\mathbf{x}}}+A_{3}}$. Here $\mu_{\mathbf{x}}$ and $\mu_{\hat{\mathbf{x}}}$ represents mean value of the images $x$ and $\hat{x}$ whereas $\sigma_{\mathbf{x}}$ and $\sigma_{\hat{\mathbf{x}}}$ represent standard deviation of the respective images. In this work, $\sigma_{\mathbf{x} \hat{\mathbf{x}}}$ represents cross-covariance between the 2-D images $\mathbf{x}$ and $\hat{\mathbf{x}} . A_{1}, A_{2}$ and $A_{3}$ are regularization constants and defined in the following way: $A_{1}=(0.01 \times[0,1]) .^{2}, A_{2}=(0.03 \times[0,1]) .^{2}$ and $A_{3}=\frac{A_{2}}{2}$. Here $[0,1]$ represents 'dynamic range' for images of type double. Exponents of value $\alpha=\beta=\gamma=1$ is used in this work. Default MATLAB function ssim(OriginalImage, ReferenceImage) has been used in this work to evaluate the structural similarity. Readers are encouraged to refer to [98] and [99] for further details about SSIM analysis.

\subsection{Database Selection}

To evaluate the performance of the modified Kronecker-based technique, MR images from the NCIGT database [89] are chosen. The database contains a total of 10 cases, each containing 903 -D slices. 2-D brain MR images of size $256 \times 256$ are sliced from 
a 3-D data sequence of $100 \mathrm{~ms}$ interval. The images are sliced using a 3-D slicing tool [100]. Randomly chosen 10 different slices, from each case (Case 1 - Case 10), are considered for evaluation.

\subsection{Results and Analysis}

From the sliced 2-D image of size $256 \times 256$, each column of the image, consisting of 256 pixels, is segmented for column-wise processing. For example, 8 segments of $n=32$ pixels each is segmented. To compress segments containing 32 pixels, measurement matrix, $\boldsymbol{\Phi}_{m \times n}$ of $m=16,8,4,2$ corresponding to respective $\mathrm{CR}=$ $50 \%, 75 \%, 87.5 \%$ and $93.75 \%$, are generated. In the recovery phase, all 8 segments are concatenated and recovered at once using the standard Kronecker-based technique. On the other hand, recovery is also performed using the ordinary CS-based technique to compare the degradation incurred by the segmentation-based technique. Discrete Cosine Transform (DCT) was used as the sparsifying basis in these comparisons. Previously mentioned smoothed $l 0$ (SL0) algorithm was used to recover the sparse signal $[46]$.

Figure 5.4 shows the comparison between the quality of reconstruction for the segmentation-based CS approach and the ordinary CS approach. The SNR values presented in this figure are average reconstruction quality obtained by averaging over the 10 cases of an arbitrarily chosen slice, $38^{\text {th }}$ slice (randomly selected from 90 slices) in this analysis. All possible segmentation for each CR is considered. For instance, for $\mathrm{CR}=87.5 \%$, the following possibilities are considered: 256 to 32,128 to 16,64 to 8,32 to 4,16 to 2 and 8 to 1 . The average reconstruction quality (quantified as $\mathrm{SNR}$ in $\mathrm{dB}$ ) is then computed for every possibility. For every given CR, the reconstruction quality of segmented CS approach will never exceed that of ordinary CS 


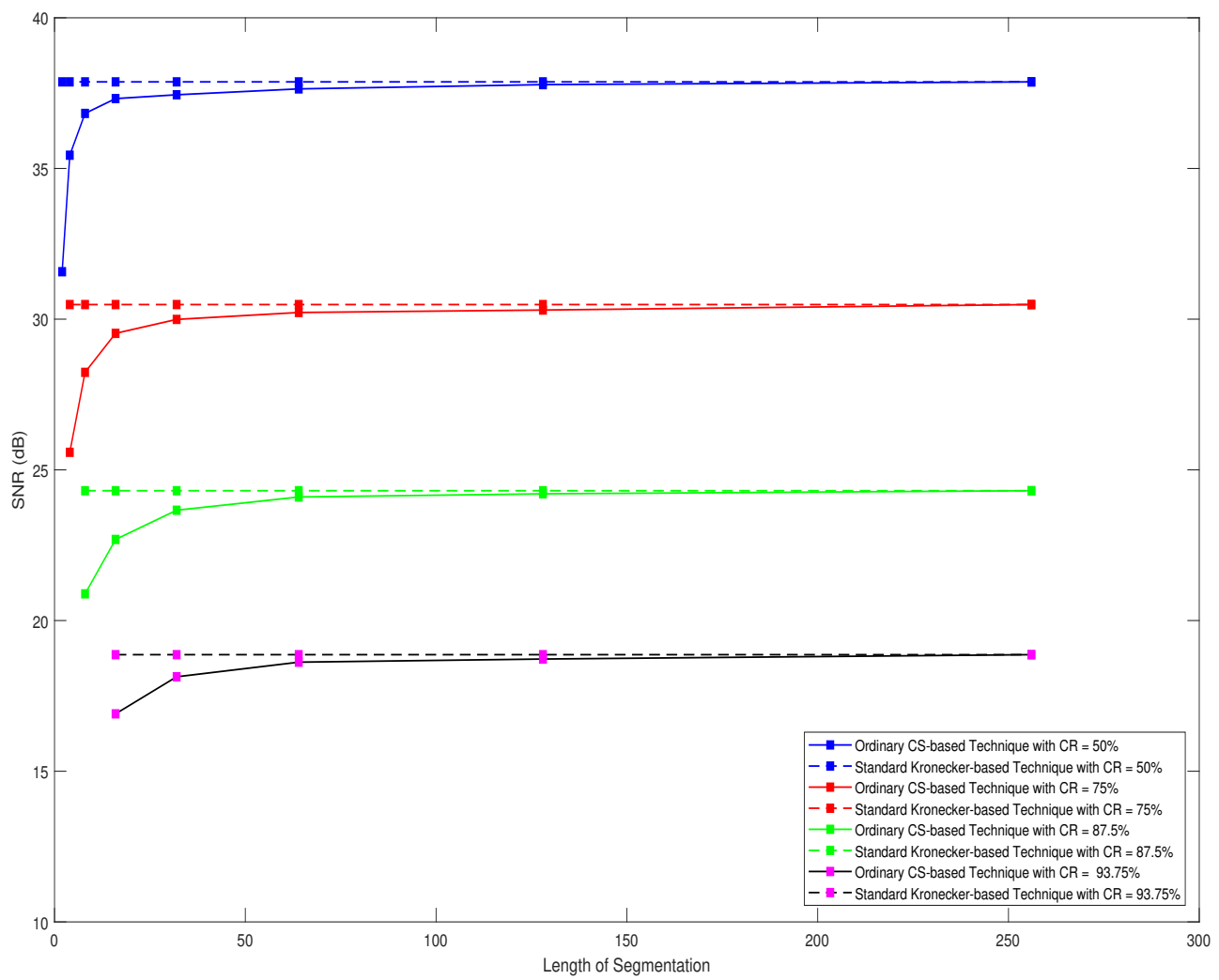

Figure 5.4: Reconstruction quality comparison between segmentation-based CS and ordinary CS methods. 
approach. The result shows that for all CRs, the reconstruction quality is almost the same as the ordinary (or non-segmentation-based) CS approach once past the knee of the curve. For higher CRs, measurement matrices of larger size are required in ordinary CS approach for maintaining the same quality of recovery when compared to segmentation-based CS method using the standard Kronecker-based recovery. Whereas in segmentation-based CS, same reconstruction quality can be achieved by using smaller measurement matrices, Furthermore, the quality obtained with $50 \%$ compression is higher than $87.5 \%$ compression, i.e. quality of the reconstructed signal reduces with increase in CR. Since there is a loss in quality of recovered images using segmented-CS approach, the modified Kronecker-based recovery is adopted for improving the quality of recovered images.

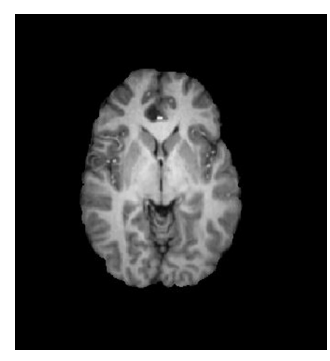

(a)

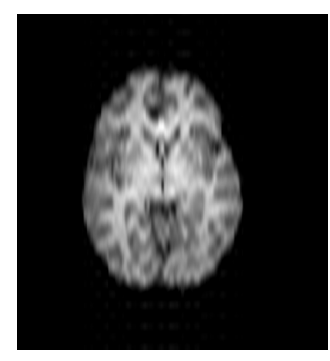

(b)

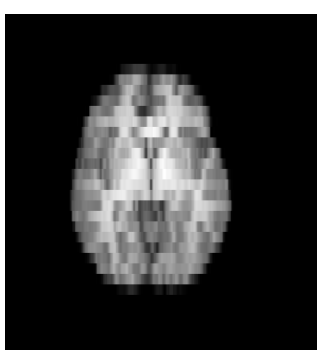

(c)

Figure 5.5: Analysis using DCT as sparsifying dictionary for $\mathrm{CR}=50 \%$ : (a) Original MR Image, (b) Recovered by the modified Kronecker-based CS recovery technique $(\mathrm{SNR}=38.99 \mathrm{~dB}),(\mathrm{c})$ Recovered by the standard Kronecker-based CS recovery technique $(\mathrm{SNR}=31.31 \mathrm{~dB})$.

Figure 5.5 - (a) is the original MR image, arbitrarily chosen $38^{\text {th }}$ slice from Case 2, while Figure $5.5-\{(\mathrm{b}) \&(\mathrm{c})\}$ are the recovered images obtained by applying the modified Kronecker-based and the standard Kronecker-based CS recovery techniques on 2-D MR image containing $256 \times 256$ pixels. DBBD matrix is used as a sensing 
matrix and DCT is the sparsifying basis at CR $=50 \%$. SL0 has been chosen as the recovery algorithm. Every column with 256 pixels was segmented into 8 segments, each having 32 pixels. Figure 5.6 shows the recovery of the MR image, arbitrarily chosen $18^{\text {th }}$ slice from Case 7 , for higher compression, i.e. $\mathrm{CR}=93.75 \%$ using DCT as a sparsifying basis. In both cases, the modified Kronecker-based technique produced a higher quality image in comparison to the standard Kronecker-based technique.

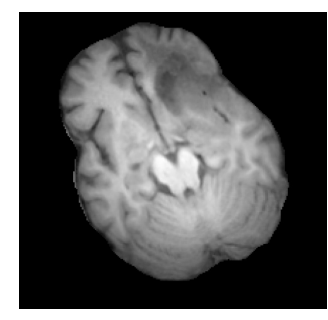

(a)

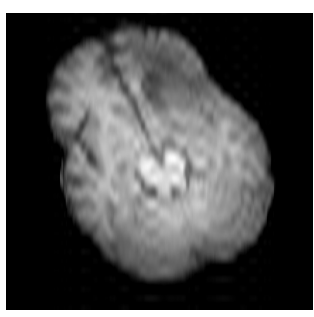

(b)

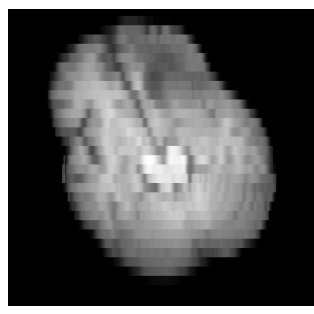

(c)

Figure 5.6: Analysis using DCT as sparsifying dictionary for CR $=93.75 \%$ : (a) Original MR Image, (b) Recovered by the modified Kronecker-based CS recovery technique (SNR $=23.05 \mathrm{~dB}$ ), (c) Recovered by the standard Kronecker-based CS recovery technique $(\mathrm{SNR}=20.74 \mathrm{~dB})$.

Both the modified and the standard Kronecker-based techniques are applied on all 10 available registered cases of the aforementioned repository. 10 slices were arbitrarily selected, from each case, and an average SNR was computed after recovery. The reconstruction quality, as a function of SNR, is presented in Table 1 - Table 10 (please see Appendices). From the results, it is clear that although the actual improvement varies from slice to slice, the improvement is always present for all CRs. These results again confirm the premise that the loss of quality caused by segmentation of the 2-D signal before compression can be countered using the modified Kronecker-based CS technique. The variations in the actual improvement are attributed to the difference in the degree of compressibility and content of the MR images.

Figure 5.7 indicates the structural similarity between the original MR image with 
that of the image recovered by both the standard and the modified Kronecker-based techniques. Aforementioned SSIM has been used as a similarity measure metric. The result is obtained by averaging the SSIM values recorded by averaging the arbitrarily selected 10 slices from all 10 cases. CR $=93.75 \%$ with DCT as a sparsifying basis has been chosen. DBBD has been used as a measurement matrix. Result clearly indicates that the similarity between the original MR image and the recovered image obtained by the modified Kronecker-based technique is consistently higher than that of the standard Kronecker-based technique, for all the 10 cases. It may be noted that the last chapter it was concluded recovery of signals obtained through deterministic framework was better than those obtained through the random measurement framework. So, it is concluded that SSIM for the recovered image using a deterministic matrix will be higher than that obtained using random matrices. Furthermore, in this thesis, only $\mathrm{CR}=93.75 \%$ is shown as this is the worst case and other CRs considered in this work will provide better SSIM.

\subsection{Summary of column-wise 2-D CS}

Discussions in the above sections address the problem stated in Section 5.1 of this chapter. An extension of the 1-D Kronecker-based CS recovery technique to 2-D signals have been presented. In order to reduce the size of the measurement matrix, a segmentation-based CS approach is introduced. The columns are segmented and each column is individually compressed (which resembles segmentation-based measurement for 1-D signals discussed in Chapter 4). Recovery of individual segments leads to loss of quality in the recovery. To improve the quality, combined or group recovery by recovering all the segments at once (the modified Kronecker-based recovery) is attempted. The proposed segmentation-based CS approach is applied to 2-D MR 


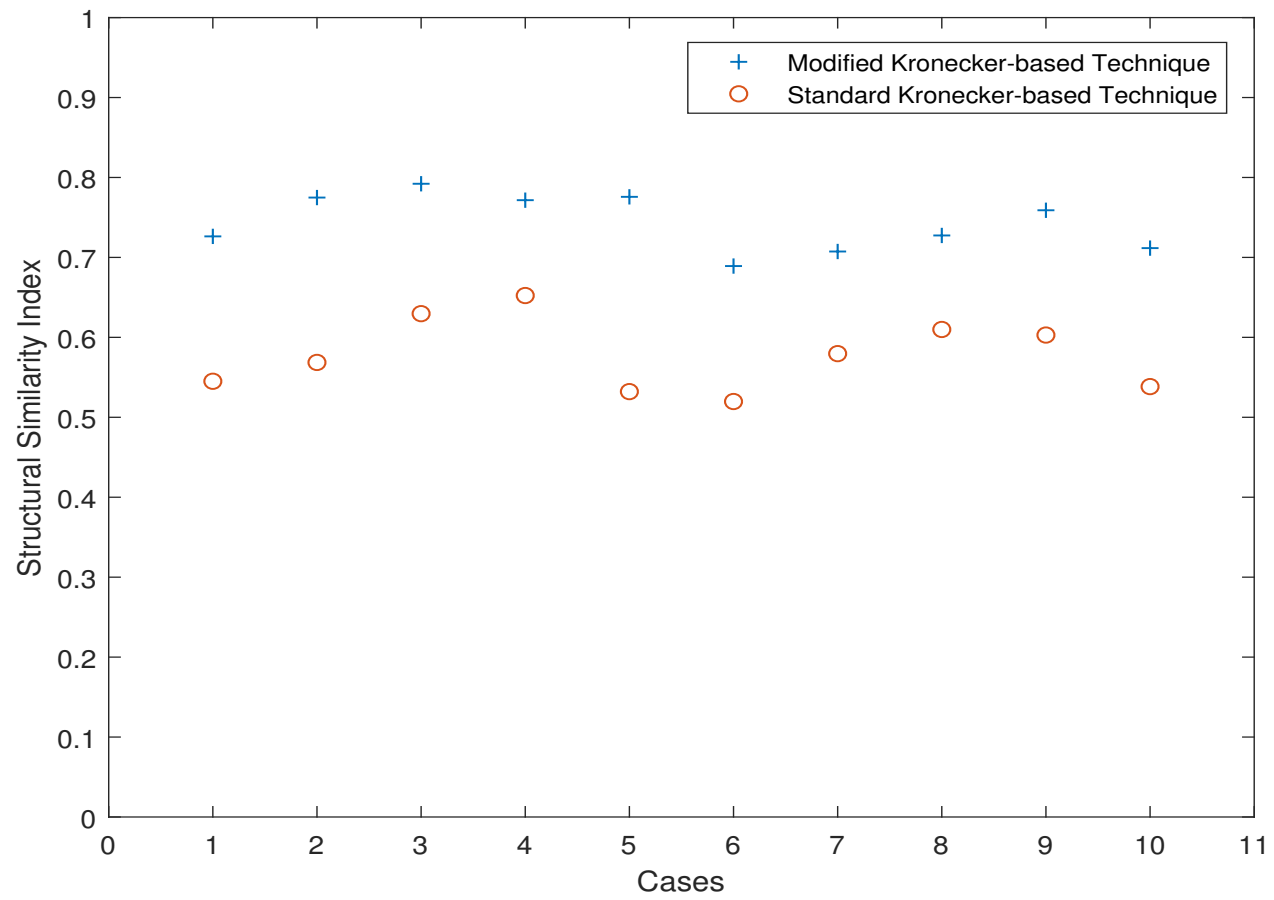

Figure 5.7: Comparison using structural similarity index for $\mathrm{CR}=93.75 \%$. 
images and the reconstruction quality is quantified and analyzed for varying CRs. For lower CRs, smaller segments of a column and hence smaller measurement matrices provide similar results like ordinary CS approach.

In the next sections of the thesis, a modified 2-D measurement technique is introduced and compared with the performance (in terms of quality of the reconstructed signals) with the column-wise CS approach, discussed in this chapter.

\subsection{Modified 2-D Compressive Sensing}

Unlike the column-wise 2-D compressive sensing, in the modified sensing approach, both the rows and columns are compressed during the measurement phase. In this thesis row \& column-wise 2-D CS technique and the modified 2-D CS has been used interchangeably.

One of the major disadvantages of the column-wise CS for 2-D signals is the aspect ratio of the measurements in the compressed domain. For example, the compressed domain representation of an image $\mathbf{X}_{N \times N}$ would be $\mathbf{Y}_{M \times N}$. Hence, the compressed domain processing (if at all chosen) of the signals, becomes difficult. Moreover, $M \times N$ number of pixels are required to be stored (in order to store the compressed images) in the column-wise CS approach. For $N$ being a large number, oftentimes the storage requirement might be impractical for many applications.

A measurement approach is introduced for 2-D signals which further reduces the storage space requirement in the compressed domain and preserves the aspect ratio. The compressed domain aspect ratio preservation may be advantageously used by signal processing applications in the compressed domain, thus avoiding computationally expensive sparse recovery.

Following the 1-D CS framework, one can consider both random and determinis- 
tic frameworks. Random matrices with elements drawn from Normal and Bernoulli distributions and DBBD deterministic matrix have been used for analysis purposes in this thesis.

In the modified 2-D CS, the $N \times N$ signal can be assumed to be decomposed in the following fashion.

$$
\mathbf{X}_{N \times N}=\mathbf{X}_{N \times N}^{0.5} \mathbf{X}_{N \times N}^{0.5}
$$

such that the energy of the 2-D signal is equally distributed in the two decomposed 2-D signals of the same size. Once the signal is decomposed into two parts, it may be further assumed that two separate measurement matrices of size $M \times N$ perform row and column-wise CS measurements. Once the row \& column-wise measurements are obtained, it may be further assumed that the two measurements are multiplied and the compressed measurement matrix $\mathbf{Y}_{M \times M}$ is obtained. The so obtained compressed measurement matrix preserves the aspect ratio. However, this is to be noted that measurements needed in the compressed domain are $M \times M$, as opposed to $N \times N$ to the column-wise CS approach.

The above explanations can be mathematically summarized as follows:

$$
\begin{aligned}
\mathbf{Y}_{M \times M} & =\boldsymbol{\Phi}_{M \times N} \mathbf{X}_{N \times N} \boldsymbol{\Phi}_{M \times N}^{T} \\
& =\left(\boldsymbol{\Phi}_{M \times N} \mathbf{X}_{N \times N}^{0.5}\right)\left(\boldsymbol{\Phi}_{M \times N}\left(\mathbf{X}_{N \times N}^{0.5}\right)^{T}\right)^{T}
\end{aligned}
$$

Figure 5.8 presents the block diagram of the modified CS approach, where the 2-D signal is compressed using row \& column-wise approach and recovered accordingly. 


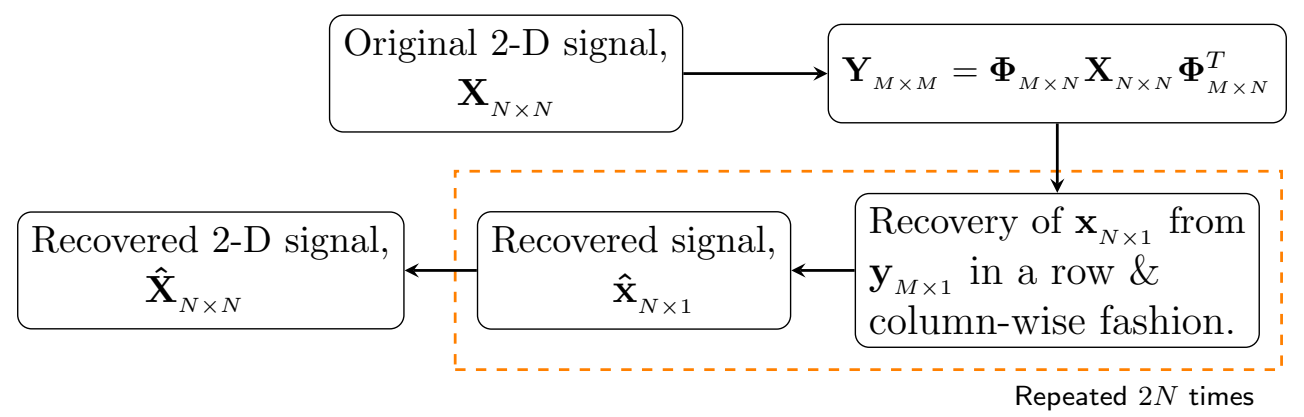

Figure 5.8: Modified 2-D CS: row \& column-wise measurement and recovery.

\subsubsection{Recovery in Modified 2-D CS}

Once the 2-D signals are measured compressively in the row \& column-wise fashion, the next task is to reconstruct the recovered signal back. The recovery is performed in the following two steps:

$$
\mathbf{Y}_{M \times N}^{\prime}=\Re\left(\mathbf{Y}_{M \times M}, \boldsymbol{\Phi}_{N \times M}^{T}, \Psi_{N \times N}^{T}\right)
$$

Here, $\mathbf{Y}_{M \times N}^{\prime}$ can be viewed as the reconstruction result of the first step which is fed to the second step as compressed measurement vector. Equation 5.6 can be viewed as row recovery whereas equation 5.7 can be treated as column recovery.

$$
\mathbf{S}_{N \times N}=\Re\left(\mathbf{Y}_{M \times N}^{\prime}, \boldsymbol{\Phi}_{M \times N}, \mathbf{\Psi}_{N \times N}\right)
$$

Figure 5.9 shows the block diagram of the row \& column-wise that is needed to be performed in order to obtain the sparse solution $\mathbf{S}_{N \times N} \cdot \ell_{0}$-norm-based fast recovery technique known as SL0 [46] is used as preferred sparse reconstruction algorithm. Reconstruction is performed $2 N$ times in the modified 2-D CS technique, as opposed to the $N$ times in the previously discussed column-wise CS approach. 


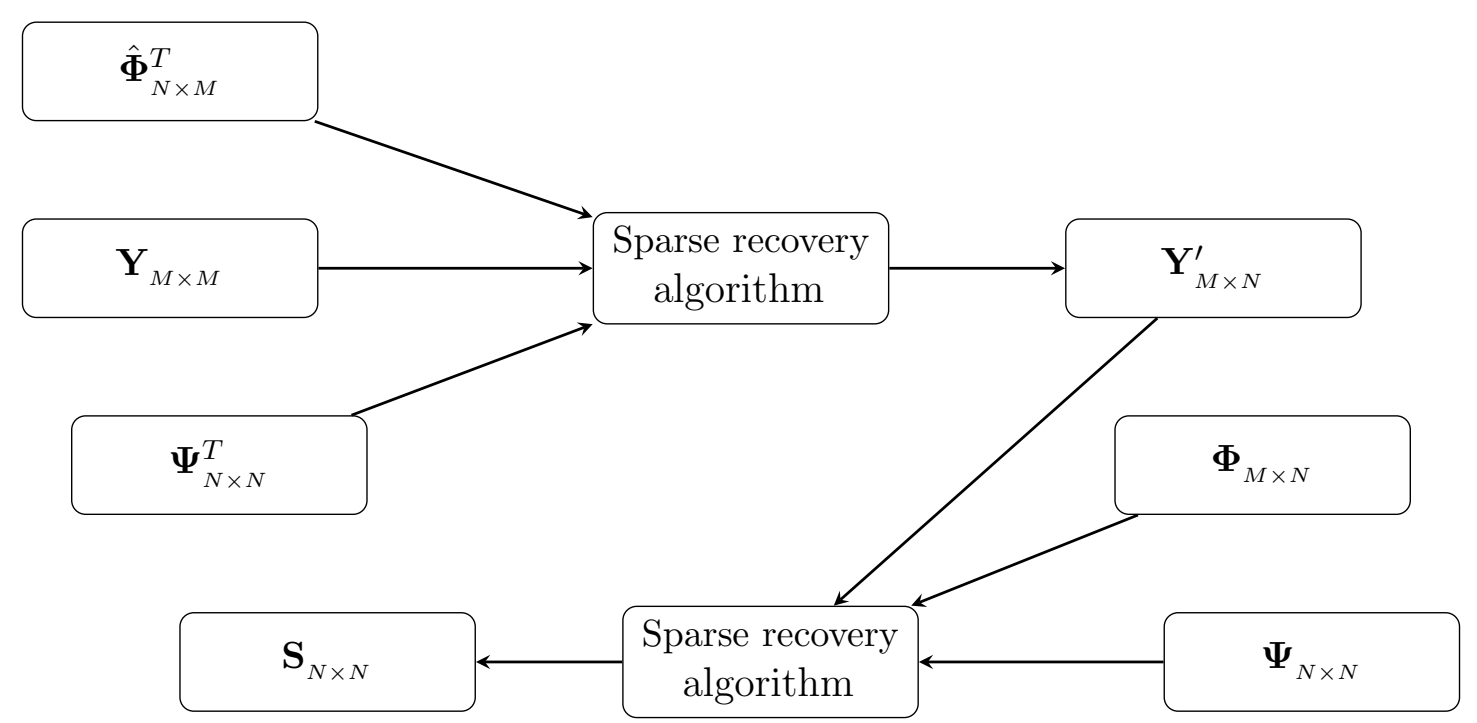

Figure 5.9: Block diagram representation of the modified 2-D CS recovery.

\subsection{Performance Analysis}

Following metrics have been used to evaluate the performance of the modified 2-D CS technique.

\subsubsection{Peak Signal-to-Noise Ratio (PSNR)}

MSE between two images, $P$ and $Q$, both of size $M \times N$ is defined as,

$$
M S E=\frac{1}{M N} \sum_{i=1}^{M} \sum_{j=1}^{N}\left(P_{i j}-Q_{i j}\right)^{2}
$$

In this thesis, PSNR is defined as follows [101]:

$$
P S N R=10 \log _{10}\left(\frac{255^{2}}{M S E}\right)
$$




\subsubsection{Structural Similarity Index (SSIM)}

The definition of SSIM follows equation 5.3 from Section 5.4.2 of this chapter.

\subsection{Database Selection}

In order to evaluate the improvement in the reconstructed signal quality of the modified Kronecker-based recovery technique with the modified 2-D CS, NCIGT database has been used [89]. The description of the database and the considered images are discussed in Section 5.5 of this chapter.

\subsection{Compression Ratio (CR)}

In this chapter, a different interpretation of $\mathrm{CR}$ is presented. This interpretation is different from the ones used in (and followed in all other sections of this thesis) Chapter 4 - Section 4.8.1. Let us consider 2-D signal of size $512 \times 512$. Based on the definition of CR in Section 4.8.1, in the column-wise 2-D CS for CR $=75 \%$ the size of the compressed measurement would be $128 \times 512$. In this case, a total of 65,536 measurements would be available in the compressed domain. The same number of measurements in the compressed domain may be obtained by simultaneously ${ }^{2}$ by applying $50 \% \mathrm{CR}$ in row \& column-wise compression. Row-wise compression of the 2-D signal would produce a matrix of size $512 \times 256$ and a further column-wise compression would produce $256 \times 256$ matrix. Hence, a total of 65, 536 measurements would be available in the compressed domain, which is equivalent to the $75 \%$ compression in column-wise CS. As a result, in the modified 2-D CS, 50\% compression both row and

\footnotetext{
${ }^{2}$ This is to be noted that in the thesis simultaneous has been used from the perspective of distributed processing. It is assumed that both row \& column-wise compression is conducted at the same time in a distributed manner.
} 
column-wise would lead to $\mathrm{CR}=75 \%$. Similarly, various other CRs can be realised.

\subsection{Result and Analysis}

In order to validate the improvement in signal recovery while using the modified 2-D CS, Images of size $256 \times 256$ are considered. In order to perform the standard or the modified Kronecker-based recovery, each column is segmented into 16 segments, each of length 16 (or 16 pixels). To compress segments containing 16 pixels, measurement matrix, $\boldsymbol{\Phi}_{m \times n}$ is chosen to be DBBD matrix. CRs of $75 \%, 87.5 \%$ and $93.75 \%$ are considered. In the recovery phase, all 16 segments are concatenated and recovered at once using both the standard and the modified Kronecker-based techniques. Dis-

crete Cosine Transform (DCT) is used as the sparsifying basis. Smoothed 10 (SL0) algorithm is used to recover the sparse signal.

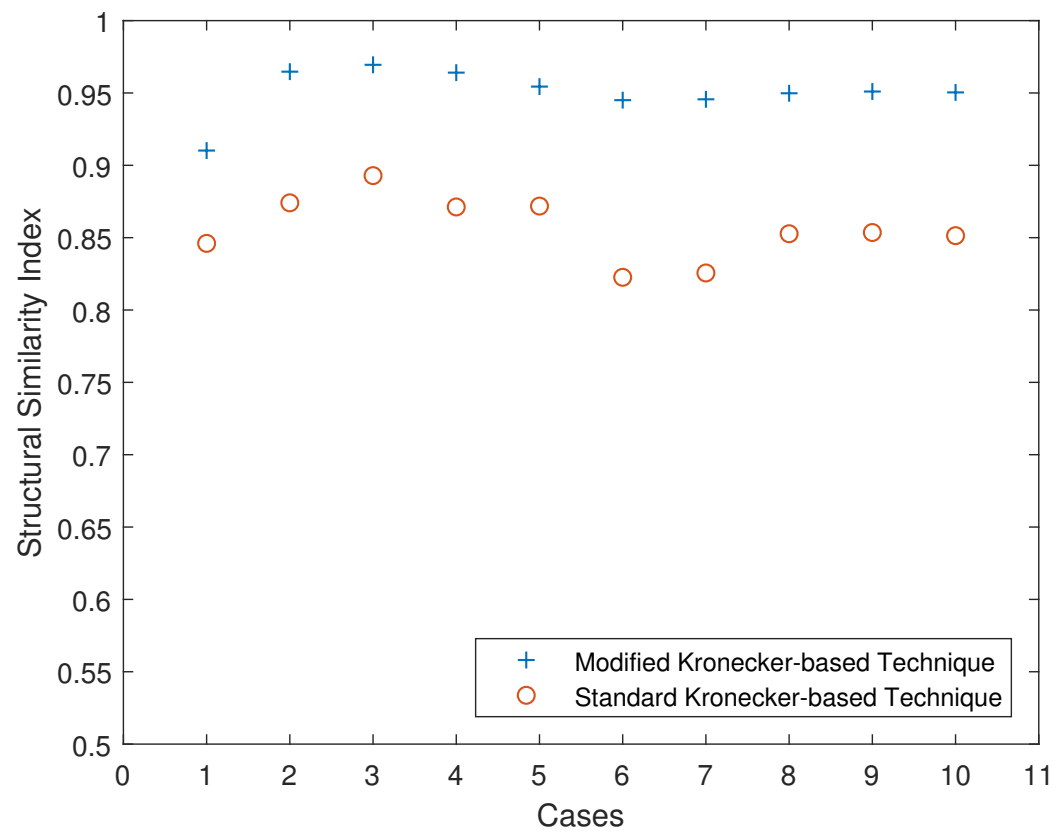

Figure 5.10: Comparison using structural similarity index for $\mathrm{CR}=75 \%$. 


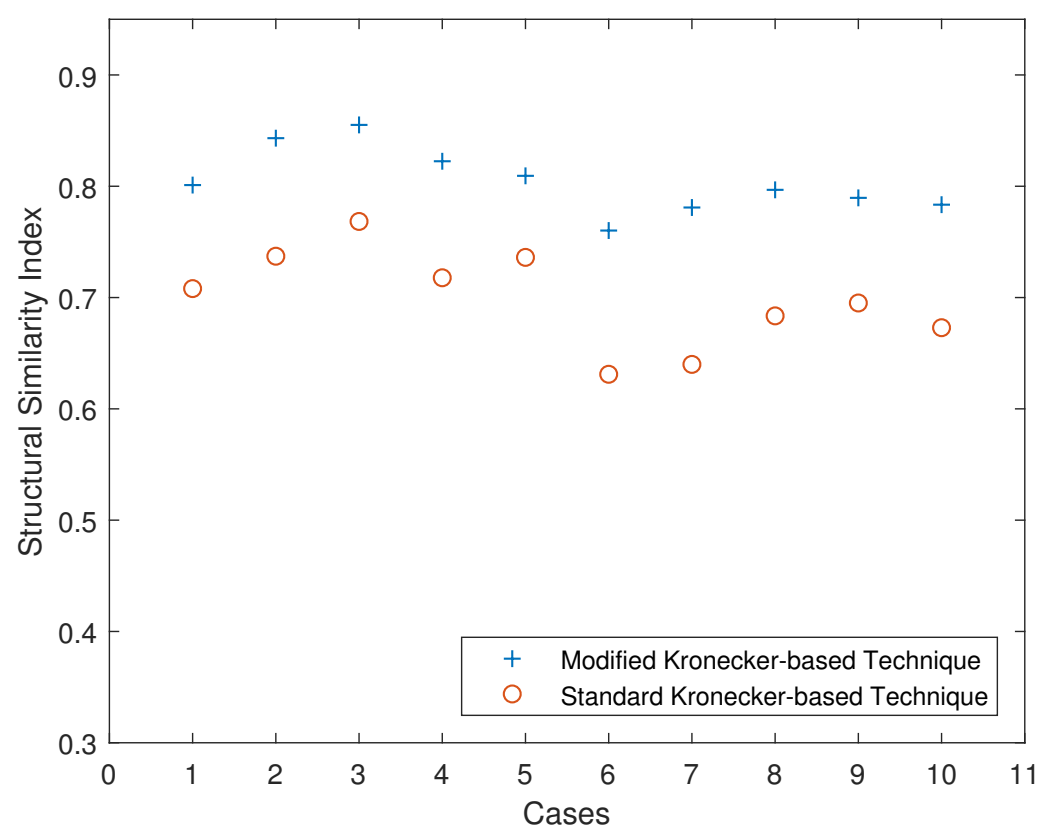

Figure 5.11: Comparison using structural similarity index for $\mathrm{CR}=87.5 \%$.

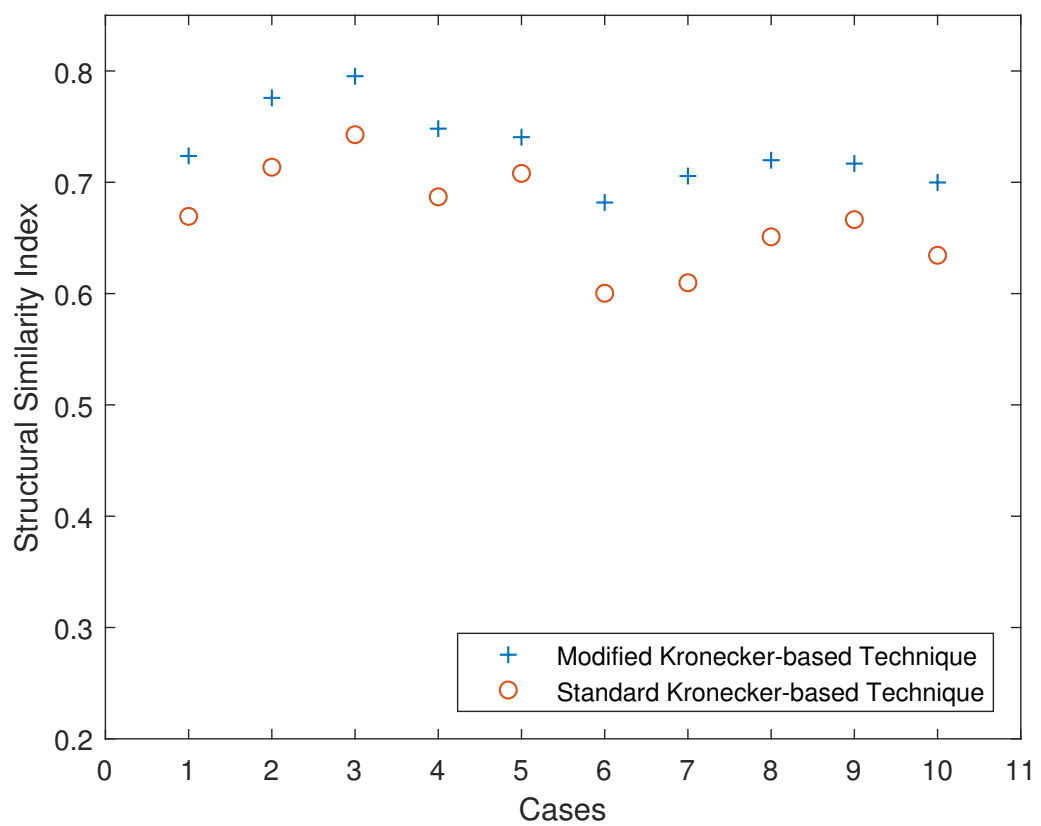

Figure 5.12: Comparison using structural similarity index for $\mathrm{CR}=93.75 \%$. 
Figures 5.10, 5.11 and 5.12 show the structural similarity analysis with $\mathrm{CR}=$ $75 \%, 87.5 \%$ and $93.75 \%$ respectively. Results are obtained by averaging the SSIM values from arbitrarily selected 10 slices from all 10 cases. Results for all the CRS indicate the improvement in reconstruction quality obtained by the modified Kronecker-based technique over the standard Kronecker-based recovery. Evidently, SSIM is higher for lower values of $\mathrm{CR}$.

The improvement in quality in terms of PSNR in $\mathrm{dB}$ is shown in Table 11 20 of Appendices. Table 21 - Table 30 presents the quality improvement attained by the modified Kronecker-based recovery technique while using random matrices with Normal distribution. Similar analysis using random matrices with Bernoulli distribution has been shown in Table 31 - Table 40 of Appendices. Analysis with the random matrices is performed by finding an average over 100 realisations. This is to be noted that, for all CRs, the modified Kronecker-based recovery technique outperformed the standard recovery technique. Results also indicate that the random matrix with elements drawn from the Normal distribution outperforms its Bernoulli counterpart for all CRs, in terms of reconstructed signal quality.

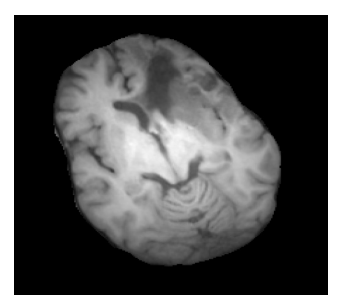

(a)

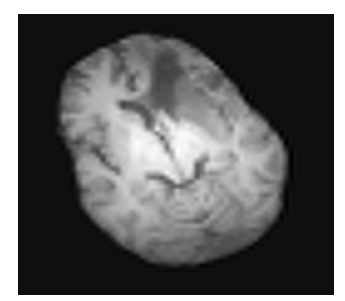

(b)

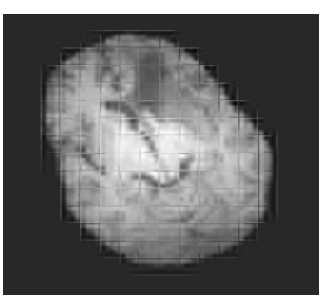

(c)

Figure 5.13: Analysis using DCT as sparsifying dictionary at CR $=93.75 \%$ for the modified 2-D CS: (a) Original MR Image, (b) Recovered by the modified Kroneckerbased CS recovery technique (PSNR $=34.89 \mathrm{~dB})$, (c) Recovered by the standard Kronecker-based CS recovery technique $(\mathrm{PSNR}=28.51 \mathrm{~dB})$.

In Figure 5.13, Slice 18 of Case 3 has been chosen to perform 2-D the modified CS 
measurement and recovery. This is to be noted that quality improvement in recovery (obtained by using the modified 2-D CS) can be seen by comparing Figure 5.6 and 5.13 .

\subsection{Summary}

In this chapter, the application of the modified Kronecker-based sparse recovery has been presented for 2-D signals (images). 1-D CS-based signal measurement and recovery framework have been extended to 2-D using two different approaches: columnwise measurement and recovery of 2-D signals and a row \& column-wise modified 2-D CS. In order to reduce the size of the measurement matrix, a segmentation-based approach has been proposed. For both the techniques, the modified Kronecker-based CS recovery technique (discussed in Chapter 3), has been used to remedy the signal quality deterioration caused by segmentation. The modified 2-D recovery technique is viewed as a two-step recovery process: a row-wise recovery followed by a column-wise recovery. Performance evaluation metrics have been introduced to quantify the improvement in reconstruction quality achieved through the modified Kronecker-based technique. Both visual (qualitative) and quantitative analysis using structural similarity index demonstrate improvement in the quality of the recovered images.

In the next chapter, the possibility of compressed domain signal processing in the compressed domain using the modified 2-D CS approach is explored. 


\section{Chapter 6}

\section{Deterministic Sensing-Based \\ Compressed Domain Image \\ Analysis $^{1}$}

In this chapter of the thesis, the possibility of compressed domain processing of 2-D signals, without the need to perform CS reconstruction is investigated. Based on the discussions of Chapter 5, the modified 2-D CS preserves the aspect ratio of the signals in the compressed domain. Hence, the compressive measurements obtained using a morphology preserving deterministic matrix is used.

Images obtained by an infra-red electro-optic camera on an airborne platform (low resolution), LandSat (medium resolution) and multispectral images (high resolution) are chosen for the modified 2-D CS. Features of chosen objects from an uncompressed image are compared with those of corresponding objects in the compressed image using template matching to demonstrate that image analysis can be done in the compressed domain. Frobenius norm-based structural similarity analysis for the images

\footnotetext{
${ }^{1}$ Contents presented in this chapter have partially been published (or submitted for publication) in $[102,103]$ by the author.
} 
at different levels of compression is presented to demonstrate the similarity in structure. Robustness of the deterministic CS technique is demonstrated through template matching using SURF algorithm.

\subsection{Introduction}

While compressing natural images, the quality of reconstruction relies on the local and non-local sparsity of the image [104]. In [105], principal component analysis (PCA) was used to adaptively decompose the image as local and non-local sparse images. By exploring the dissimilarity between the sparse components, adaptive sensing models were proposed in $[104,106]$. By imposing more computational burden during the encoding process, the simplicity of the encoding process is lost. Even if the low complexity encoding (measurement) process is maintained, these CS-based techniques for image compression have high computational complexity while decoding (reconstruction). Because of the heavy computational demand during reconstruction, it may be worthwhile to conduct signal or image processing in the compressed domain wherever possible without resorting to reconstruction and this paper investigates such an idea.

A dimensionally reduced matched filter to perform detection and object tracking application on radar images without performing the computationally expensive reconstruction process is proposed in [16]. In this work, a random measurement matrix has been used for compression purposes. Although several studies have appeared in the literature, where analysis have been proposed in the compressed domain [107-109] there has been no attempt to exploit simple and easy-to-implement deterministic sensing approach for obtaining compressed images. Also, there has been not much attempt to conduct image analyses in the compressed domain either. 
In this chapter, the modified 2D deterministic sensing technique (discussed in Chapter 5) for compressing an image is considered. Linear filtering-based deterministic matrix, DBBD, that preserves morphology of the image has been chosen for this study [35] (also see Chapter 2 - Section 2.4.2). As discussed in the previous chapter, the modified 2-D CS-based measurement maintains the aspect ratio of the image in the compressed domain. In conjunction with the linear filtering-based deterministic measurement, the structural similarity of the images are maintained in the compressed domain. Frobenius norm is used as a metric to demonstrate structural similarity while the objects in the compressed and uncompressed images are compared using a feature-based technique. Our analysis demonstrates that the significant features of the image remain intact in the compressed domain even when noise is present.

The novelty of the previously mentioned linear-filtering-based modified 2-D CS approach is as follows. The deterministic CS technique is applied to images which compresses the image both row and column-wise 'simultaneously' preserving the aspect ratio and the structural similarities of objects in the compressed image. Such

a technique has not been reported earlier in the literature. The modified 2-D CS approach using deterministic sensing may preserve structure and features in clean and noisy compressed images.

\subsection{2-D Deterministic Measurement and Recovery}

In order to perform the row \& column-wise measurement (to preserve the aspect ratio of the images in the compressed domain), the modified 2-D CS technique introduced in Chapter 5 - Section 5.8 is adopted. Compressed domain image is obtained following the steps shown in Figure 5.8. DBBD matrix is used as a deterministic mea- 
surement matrix, which enables easy implementation and morphology preservation in the compressed domain. For some applications, if the original image is required to be recovered, the 2-step recovery process described in Chapter 5 - Section 5.8 can be adopted.

\subsection{Feature-Based Template Matching}

A feature-based template matching approach is undertaken to demonstrate compressed domain feature preservation. Templates are formed from the original uncompressed images and a match is obtained in the compressed images. This approach also further validates the preservation of significant features in the compressed image obtained from deterministic compressive sensing, thus enabling analysis of the compressively sampled image in the compressed domain itself.

In the literature, a variety of feature extraction algorithms are proposed [110-113]. A scale-invariant feature transform (SIFT) algorithm to combine local descriptors with interest points by allowing repeatable and reliable measurement from images was presented in [114] and was considered a robust technique by experts till speeded up robust features (SURF) was proposed in [115] to encompass scale and rotation invariant detector and descriptor for applications like object recognition from images. In this work, the SURF algorithm is chosen because of its faster and robust performance over SIFT.

In SURF, points of correspondences between the uncompressed and the compressed images are sought after. This is achieved using the following three steps: a) Points which are distinctive in the images are chosen and termed as 'interest points' (generally blob-like structures in the images). b) The neighborhood of every interest point is considered as a feature vector and are assumed to be invariant to deforma- 
tions and robust to noise, and c) Feature vectors are then matched using a distance criterion.

As the interest points have to be scale-invariant, Hessian-based detection technique [116] is used. Since the calculation of Hessian is computationally expensive, approximations like the Difference of Gaussian (DoG) is preferred to make the implementation faster. In order to detect interest points, integral images are used. An integral image at position $(x, y)$ is represented by $\mathbf{I}_{\Sigma}(x, y)$ and defined by (Equation 6.1) the summation of all pixels in a rectangular area formed by origin and $(x, y)$.

$$
\mathbf{I}_{\Sigma}(x, y)=\sum_{i=0}^{\leq x} \sum_{j=0}^{\leq y} \mathbf{I}(i, j)
$$

Once the integral images are evaluated, a Fast-Hessian approach is used to identify significant features. Hessian matrix for a point $(x, y)$ in an image $\mathbf{I}(x, y)$ is defined as,

$$
\mathcal{H}(x, y, \sigma)=\left[\begin{array}{cc}
\frac{\partial^{2} G(\sigma)}{\partial x^{2}} * \mathbf{I} & \frac{\partial^{2} G(\sigma)}{\partial x y} * \mathbf{I} \\
\frac{\partial^{2} G(\sigma)}{\partial x y} * \mathbf{I} & \frac{\partial^{2} G(\sigma)}{\partial y^{2}} * \mathbf{I}
\end{array}\right]
$$

But, the approach depicted in Equation 6.2 introduces aliasing while using Gaussian filters for sub-sampled images. To avoid that, in the fast-Hessian matrix approach, convolution ( denoted by ${ }^{(*)}$ ) operation between $\mathbf{I}$ and a 'box filter approximation' ( $D_{x x}, D_{x y}$ and $D_{y y}$ ) of Gaussian kernel is performed. Equation 6.2 is modified in the following way,

$$
\mathcal{H}^{\prime}(k)=D_{x x}(k) D_{y y}(k)-\left(0.9 D_{x y}(k)\right)^{2}
$$

where $k=(x, y, \sigma)$. Note that $\sigma$ denotes the width of the Gaussian filter. Local maximas are calculated from the determinant of $\mathcal{H}^{\prime}(k), \operatorname{det}\left|\mathcal{H}^{\prime}(k)\right|$, and are identified 
as the significant features or 'detectors'.

Once the significant features are identified, 'descriptors' are identified. In [115], 'descriptors' are defined by the distribution of Haar wavelet response (to ensure 'lighting invariance') in the neighbourhood of 'detectors'. Haar wavelets may be thought of providing a directional derivative of the intensity of images. Haar wavelet response is calculated along the $\mathrm{x}, \mathrm{y}$-axis and a circular neighbourhood of radius $6 \sigma$. Wavelet responses for multiple $\sigma$ s are calculated, to ensure scale invariance. Selection criteria of $\sigma \mathrm{s}$ are discussed in [117]. The responses are centered around the 'detectors' and summed up in a sliding window technique (covering $\frac{\pi}{3}$ ) to obtain 'descriptors'.

To identify a particular object from a compressed image, SURF features and associated 'descriptor vectors' are evaluated from both the template (pre-chosen object in the uncompressed image) and the compressed image. Feature points are considered as a 'match' if the Euclidean distance between two descriptors is less than 0.7 times to that of the second nearest neighbour [110].

A histogram-based inlier selection technique using affine transform model, proposed in [118], is used to remove outliers from the feature points. The inliers in the template are mapped onto the inliers in the compressed image, after removing outliers using the MSAC algorithm [119]. Finally, a bounding box is plotted to identify the location of the template in the compressed image.

\subsection{Database Selection}

For this work, three different types of images: infra-red, high resolution and medium resolution are considered. The details of the data set are as follows: 


\subsubsection{Low Resolution Image}

Infra-red images are acquired using a commercial EO/IR sensor on an airborne platform. Images are obtained using the MX-15 IR camera system installed on NRC Convair 580 airborne platform [120].

\subsubsection{Medium Resolution Image}

Landsat medium resolution images are considered. Images contain 9 spectral bands and have a spatial resolution of 30 meters. Images acquired by Landsat 8 OLI sensor, having a spatial resolution of 15 meters, has been used for analysis. Images used in this analysis are acquired on a scale of 1:289K over the region of Ottawa, Canada. This data has been made freely available by United States Geological Survey [121,122].

\subsubsection{High Resolution Image}

High resolution images acquired by Digital Globe's 8-band multi-spectral commercial satellite, WorldView-2 are also considered. The satellite operates at $770 \mathrm{~km}$ altitude maintaining an inclination angle of $97.2^{\circ} \quad[123]$.

\subsection{Results and Analysis}

To validate the performance of $2 \mathrm{D}$ deterministic sensing technique, two types of analyses are performed: structural similarity analysis and compressed domain feature preservation analysis. In this work, compression ratio (CR) is defined as $C R(\%)=\left(1-\frac{M}{N}\right) \times 100 \%$. For these analyses, $\mathrm{CR}=50 \%$ and $75 \%$ are considered. Please note that the definition of $\mathrm{CR}$ followed in this chapter is different from that of the previous chapter (refer to Chapter 5 - Section 5.11). 


\subsubsection{Structural Similarity Analysis}

To quantify the structural similarity between the original uncompressed image and compressed images, matrix norm evaluation is undertaken. Since most of the information is preserved during the deterministic compressive sensing, eigenvectors and associated eigenvalues remain almost unchanged. A matrix norm-based on eigenvalue: Schatten $p-$ norm is a good candidate to demonstrate the structural similarity between the compressed and uncompressed images. Schatten norm for $p=2$ turns out to be Frobenius norm [124]. Frobenius norm for a matrix $A_{a \times b}$ is defined as follows,

$$
\|A\|_{F}=\sqrt{\sum_{i=1}^{a} \sum_{j=1}^{b}\left|A_{i, j}\right|^{2}}
$$

For $A$ being real, eq. 6.4 can further simplified as $\|A\|_{F}=\sqrt{\operatorname{Trace}\left(A^{T} A\right)}$.

Results in Table 6.1 records the Frobenius norm calculated for three different types of images at two different compression levels: $\mathrm{CR}=50 \%$ and $75 \%$. An analysis is performed on 10 test images for different resolutions from the above-mentioned database and average results are reported. Similar analysis has been performed on the recovered images using the modified Kronecker technique (shown in Table 6.2), obtained by sparse recovery of the compressed images for both the CRs. Smooth $\ell_{0}$ algorithm has been used to perform CS recovery $[46,88]$. From the results, it is clear that the Frobenius norm of both compressed and recovered images are very close to that of the original image. Hence, it may be inferred that the structural similarity has been maintained through the deterministic sensing technique.

Figure 6.1 shows the visual representation of images (randomly chosen from the 10 test images) at different CRs, for $50 \%$ and $75 \%$. Visual similarity can be noticed in infra-red, medium and high resolution images for both the CRs. This is to be noted 
Table 6.1: Structural Similarity Analysis (Compressed Images)

\begin{tabular}{|c||ccc|}
\hline Image Type & Frobenius Norm \\
& Original Image & $\mathrm{CR}=50 \%$ & $\mathrm{CR}=75 \%$ \\
\hline infra-red & 389.04 & 388.84 & 388.59 \\
\hline medium resolution & 279.59 & 279.57 & 279.49 \\
\hline high resolution & 452.37 & 452.02 & 450.81 \\
\hline
\end{tabular}

Table 6.2: Structural Similarity Analysis (Recovered Images)

\begin{tabular}{|c|c|c|c|}
\hline \multirow[t]{2}{*}{ Image Type } & \multicolumn{3}{|c|}{ Frobenius Norm } \\
\hline & Original Image & $\mathrm{CR}=50 \%$ & $\mathrm{CR}=75 \%$ \\
\hline infra-red & 389.04 & 390.39 & 389.03 \\
\hline medium resolution & 279.59 & 280.31 & 279.8 \\
\hline high resolution & 452.37 & 452.16 & 450.83 \\
\hline
\end{tabular}

that the analysis is performed on multiple images from each class of resolution and visual analysis for only one image from each class of resolution is presented, because of space constraint.

\subsubsection{Compressed Domain Feature Preservation}

To validate the notion of significant feature preservation in the compressed images, a feature-based template matching using SURF algorithm is performed. Template from an original uncompressed image is formed. The original image is then compressed using the 2-D deterministic sensing technique. SURF descriptors are extracted from both the compressed image and the template. If the position of the template can be identified in the compressed image, using the feature-based template matching technique discussed in Section 6.3, it is inferred that the significant features remain intact while using the deterministic sensing technique.

In the analysis, template matching is performed on the compressed domain (at $\mathrm{CR}=50 \%$ ) for infra-red, medium and high resolution images. In this analysis, the template of a ship is formed from the infra-red image and matching is performed 
(A)

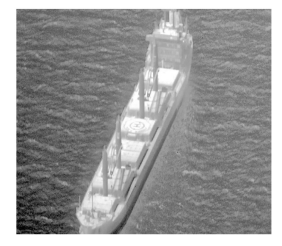

(A)

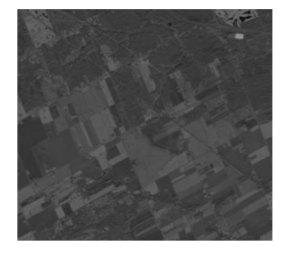

(b) Medium resolution image
(B)

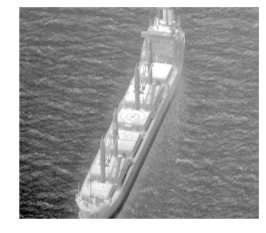

(a) infra-red image

(B)

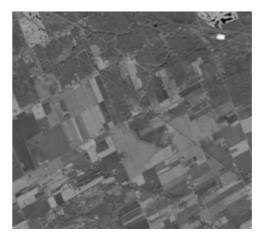

(B)

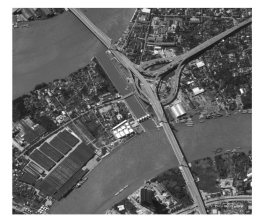

(C)

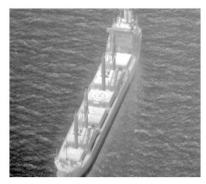

(C)

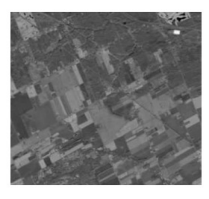

(C)

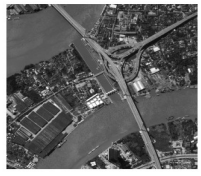

(c) High resolution image

Figure 6.1: Visual representation of images at different compression levels. (A) Original image, (B) Image with $\mathrm{CR}=50 \%$, (C) Image with $\mathrm{CR}=75 \%$. 
(A)
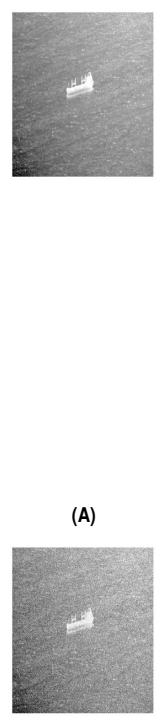

(B)

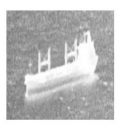

(D)

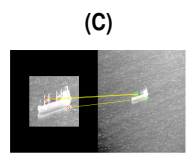

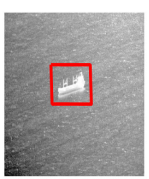

(a) Infra-red image in absence of noise.

(b) Infra-red image affected by additive Gaussian noise of $\mu=0$ and var $=0.03$.

Figure 6.2: Feature-based template matching from compressed infra-red images. (A) Original image compressed at $\mathrm{CR}=50 \%$. (B) Template generated from uncompressed image. (C) Outlier removed match point identification using SURF algorithm. (D) Bounding box indicating the position of the template in the compressed image. 

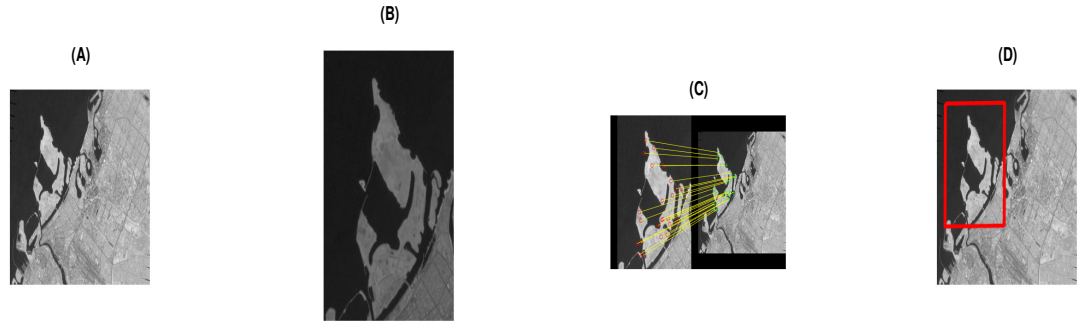

(a) Medium resolution image in absence of noise.

(B)

(A)

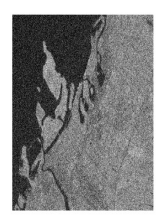

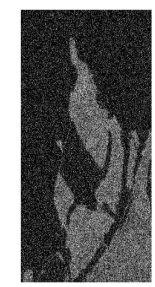

(D)

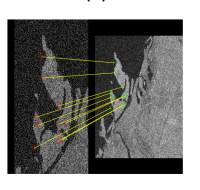

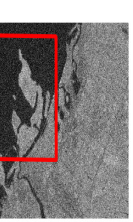

(b) Medium resolution image affected by additive Gaussian noise of $\mu=0$ and $v a r=0.03$.

Figure 6.3: Feature-based template matching from compressed medium resolution images. (A) Original image compressed at $\mathrm{CR}=50 \%$. (B) Template generated from uncompressed image. (C) Outlier removed match point identification using SURF algorithm. (D) Bounding box indicating the position of the template in the compressed image. 

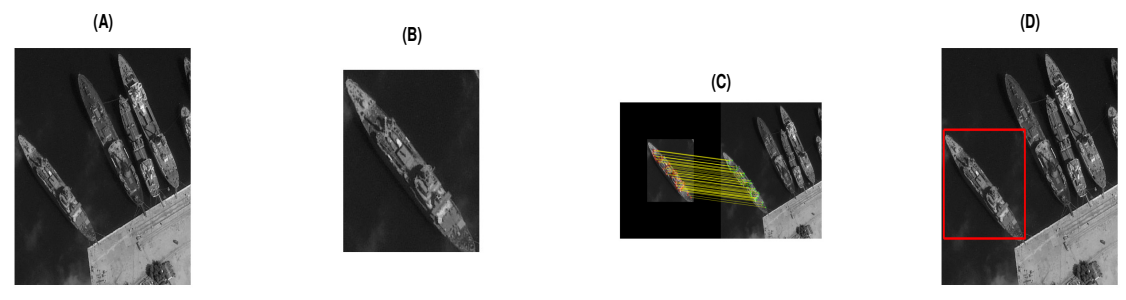

(a) High resolution image in absence of noise.

(A)

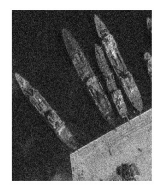

(B)

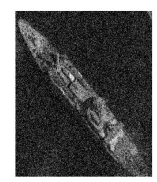

(c)

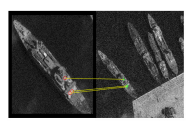

(D)

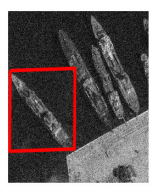

(b) High resolution image affected by additive Gaussian noise of $\mu=0$ and var $=0.03$.

Figure 6.4: Feature-based template matching from compressed high resolution images. (A) Original image compressed at $\mathrm{CR}=50 \%$. (B) Template generated from uncompressed image. (C) Outlier removed match point identification using SURF algorithm. (D) Bounding box indicating the position of the template in the compressed image. 
with the compressed infra-red image. Results are presented in Figure $6.2-\{(\mathrm{a}) \&$ (b)\}. Figure 6.2 -(a) shows the matching of the ship template with the compressed image in the absence of noise. Additive Gaussian noise of $\mu=0$ and var $=0.03$ has been added to the original image (before performing the 2-D compression) to perform the template matching, and this is shown in Figure 6.2 - (b). By performing similar operations in medium and high resolution images, templates of an island and a ship have been matched onto the corresponding noiseless and noisy compressed images from respective resolutions. From these analyses, it may be concluded that significant features are preserved in the compressed domain. Thus, image analysis may be performed in the compressive domain without resorting to computationally expensive sparse recovery.

\subsection{Summary}

In this chapter, to avoid the computationally intensive sparse recovery of compressed images prior to image analysis, a deterministic sensing-based compressed domain analysis of images are demonstrated. A morphology preserving, easily implementable linear-filtering-based deterministic matrix is utilized as a measurement matrix. The 1D deterministic CS idea is thus expanded to 2-D, using the modified 2-D CS technique introduced in Chapter 5. The proposed 2-D deterministic sensing technique preserves the aspect ratio (in the compressed domain) unlike the traditional column-wise image compression technique and hence is used for compressing the images. The proposed sensing technique is applied on several images with high, medium and low resolutions. The significant features in the compressed images are preserved in the compressed domain. Using a feature-based template matching technique, where the templates are formed from the uncompressed images and the match is found in the compressed 
images. Compressed domain image analysis demonstrates that image analyses can be performed in the compressed domain and is robust to noise. 


\section{Chapter 7}

\section{Conclusions, Limitations and}

\section{Future Works}

Continuous signal acquisition and transmission to a remote system for 'processing on demand' is a new trend in modern signal processing. In recent days, CS has been chosen as a signal acquisition technique to reduce the number of samples acquired by the sensor. In order to reduce the associated data transmission cost, measurements are acquired as smaller segments for transmission, which reduces the quality of the signal while performing reconstruction at the remote system. In this thesis, a Kronecker-based recovery solution is proposed to improve the quality of the reconstructed signal. The quality improvement is achieved through the reduction of mutual coherence between the measurement matrix expanded through the Kronecker product and the newly generated orthonormal sparsifying matrix. The performance improvement of this modified Kronecker-based technique is analyzed in 1-D by applying it on a variety of ECG signals acquired from the MIT-BIH Arrhythmia database. The proposed 1-D Kronecker-based recovery is extended to 2-D signals. A novel way

of compressing 2-D images that preserves aspect ratio is also developed and applied 
to various images. The modified Kronecker-based CS recovery is also applied to aspect ratio preserved compressed images. Thus, the thesis contributes to 1-D and 2-D signal processing.

As part of 1-D signal processing, the loss of signal quality associated with segmentation-based CS for continuous monitoring of bio-signal, such as ECG, has been addressed. A detailed investigation of the proposed modified Kronecker-based technique has been performed for various CRs and sparsifying basis. Signal quality analysis of the recovered ECG signals have been performed for both random and deterministic measurement frameworks. An easily implementable linear filtering-based deterministic measurement matrix, DBBD is considered. This measurement framework is observed to preserve the morphology of the ECG signal, in the compressed domain. Application specific inferences have been drawn on the selection of a particular measurement framework. Inferences have been made on the selection of measurement matrix framework in presence of noise (at source) by performing visual reconstruction analysis on an ECG signal, from the MIT-BIH Arrhythmia database, representing a pathological case.

Two different CS approaches for 2-D signals are considered in this work: traditional column-wise 2-D CS and a novel modified 2-D CS (or row \& column-wise 2-D CS). Novel extensions of the Kronecker-based recovery technique (originally developed for 1-D signals) to images are presented. In the column-wise approach, individual columns of the measurements are considered as 1-D vectors whereas in the modified 2-D CS, row \& column-wise measurements have been adopted. The modified 2-D $\mathrm{CS}$, row \& column-wise measurement preserves the aspect ratio (of the compressed measurements) while the column-wise 2-D CS does not. For these two 2-D CS approaches, the standard Kronecker-based recovery and the modified Kronecker-based recovery have been attempted. The modified Kronecker-based recovery technique for 
simultaneous row \& column-wise measurement is a novel extension. Statistical analysis has been performed on the NCIGT MRI database with structural similarity and SNR-based reconstruction quality indices. The results confirm improvement in signal recovery while using the modified Kronecker-based CS reconstruction technique.

This thesis further exploits the aspect ratio preserving row \& column-wise 2-D CS technique using morphology preserving deterministic sensing framework for conducting compressed domain image processing. Frobenius norm-based structural similarity analysis is performed on images of different quality, like low, medium and high resolution images. Feature-based template matching has been applied on images, of various resolutions, for multiple CRs in both noisy and noiseless images to demonstrate the feasibility of image processing in the compressed domain without the need for computationally intensive recovery.

\subsection{Limitations and Scope of Future Works}

In this work, an assumption has been made that all the signals are compressively acquired and the processing is performed in the digital domain. Future study would include a hardware-based implementation of Kronecker-based CS recovery and acquisition of 1-D signals, such as ECG. A theoretical bound should be derived for improvement that can be attained with the proposed modified Kronecker-based CS recovery technique. Although the notion of reduction in energy requirement during transmission of the data packets to a remote system can be intuitively understood, collection of analytical data in support of the notion has been out of scope of this thesis. As a part of the future work, theoretical and analytical observation would be performed quantifying the reduction in energy requirement by the transmission nodes of the sensors. A possibility of signal de-noising using the Kronecker-based technique 
is also a possible direction for future study.

Considering Kronecker-based technique for 2-D signals, an interesting pattern in signal quality improvement (as a function of PSNR in dB) has been noticed while changing CRs. The relationship connecting the optimum CR, the measurement matrix and required signal quality may provide better insight while making design choices such as CR or reconstructed signal quality and required memory storage. A detailed study for 1-D and 2-D signal with a variety of deterministic, structured random, repeated block diagonal and pseudo-random measurement matrices can be conducted for various CRs as an extension to this work.

While considering the 2-D CS-based measurement, in this thesis, the correlation information between the columns of the signal is not considered. Such considerations may lead to block-based CS for 2-D signals, where the signals are first classified into compressible and non-compressible blocks and then an effective measurement matrix is designed for each compressible block. Subsequently, block-based recovery is performed to obtain a sparse solution. Future study can focus on exploring the implementation of the modified Kronecker-based recovery technique for such blockbased 2-D CS approach.

The main focus of the thesis is on the Kronecker-based recovery for 1-D and 2D signals. However, the possibility of Kronecker-based measurement is not explored. Future work may include Kronecker-based measurement for multi-dimensional signals, such as 3-D MR image and recovery may be attempted using the modified Kroneckerbased CS recovery technique.

Although DBBD measurement matrix is identified as morphology preserving matrix, no signal processing of the ECG signal is carried out in the compressed domain. There is a possibility of developing new signal processing techniques suitable for the compressed domain signal analysis. Such techniques may be suitable for IoT devices 
when CS is used to acquire ECG for continuous monitoring. When an anomaly is detected, then the signal can be transferred to a health-care professional for further analysis.

This thesis emphasises on using deterministic compressive sensing using morphology preserving DBBD matrix. A morphology preserving compressive sensing approach might not be suitable for applications that require to preserve privacy. As random matrices do offer 'very weak' encoding of the acquired signal during compression, it may be wise not to recover the signal in the remote centre such as a cloud. Instead, a scrambled version of the signal may be recovered while true signal recovery happens only at the end user's site. This will require the use of ciphers and an extension of this thesis with security and privacy in mind would be well-worth a consideration.

In defence applications for enhancing situational awareness say in maritime environments, passive sensors such electro-optic sensors are used in air-borne surveillance vehicles such as an unmanned aerial vehicle (UAV). Since objects of interest are not present in every frame, an intelligent way of conducting timely analysis is in order. Proposed 2-D compressive sensing scheme may have utility as the proposed approach is amenable to real-time and parallel processing techniques. Machine learning techniques may be employed to detect, classify, geo-locate and track objects in the maritime environment. 
Appendices 
Results related to the analysis performed with 1-D ECG signals (discussed in Chapter 4) are available in: https://drive.google.com/drive/folders/ 1093Zk674mB3dW_ra3oPg0V1IMIteCE2g?usp=sharing

Table 1: Reconstruction Quality Analysis: Case 1

\begin{tabular}{|c|c|c|c|}
\hline \multirow[t]{2}{*}{$\mathrm{CR}(\%)$} & \multicolumn{3}{|c|}{ SNR (dB) } \\
\hline & Modified & Standard & Improvement \\
\hline 50 & 33.45 & 29.41 & 4.03 \\
\hline 75 & 30.14 & 24.16 & 5.87 \\
\hline 87.5 & 23.19 & 19.39 & 3.79 \\
\hline 93.75 & 16.83 & 15.32 & 1.50 \\
\hline
\end{tabular}

Table 2: Reconstruction Quality Analysis: Case 2

\begin{tabular}{|c||ccc|}
\hline CR (\%) & SNR (dB) & \\
& Modified & Standard & Improvement \\
\hline 50 & 35.23 & 31.59 & 3.64 \\
\hline 75 & 31.63 & 26.36 & 5.27 \\
\hline 87.5 & 26.61 & 21.50 & 5.11 \\
\hline 93.75 & 19.89 & 17.07 & 2.81 \\
\hline
\end{tabular}

Table 3: Reconstruction Quality Analysis: Case 3

\begin{tabular}{|c|c|c|c|}
\hline \multirow[t]{2}{*}{ CR (\%) } & \multicolumn{3}{|c|}{ SNR $(d B)$} \\
\hline & Modified & Standard & Improvement \\
\hline 50 & 34.70 & 30.83 & 3.87 \\
\hline 75 & 31.53 & 25.27 & 6.26 \\
\hline 87.5 & 25.11 & 20.25 & 4.86 \\
\hline 93.75 & 17.83 & 15.79 & 2.04 \\
\hline
\end{tabular}


Table 4: Reconstruction Quality Analysis: Case 4

\begin{tabular}{|c|c|c|c|}
\hline \multirow[t]{2}{*}{ CR (\%) } & \multicolumn{3}{|c|}{ SNR (dB) } \\
\hline & Modified & Standard & Improvement \\
\hline 50 & 36.15 & 32.51 & 3.63 \\
\hline 75 & 32.04 & 26.76 & 5.27 \\
\hline 87.5 & 26.05 & 21.99 & 4.05 \\
\hline 93.75 & 19.71 & 17.82 & 1.89 \\
\hline
\end{tabular}

Table 5: Reconstruction Quality Analysis: Case 5

\begin{tabular}{|c|c|c|c|}
\hline \multirow[t]{2}{*}{ CR (\%) } & \multicolumn{3}{|c|}{ SNR $(\mathrm{dB})$} \\
\hline & Modified & Standard & Improvement \\
\hline 50 & 31.14 & 28.41 & 2.73 \\
\hline 75 & 26.99 & 23.64 & 3.34 \\
\hline 87.5 & 22.70 & 19.62 & 3.07 \\
\hline 93.75 & 17.66 & 15.72 & 1.94 \\
\hline
\end{tabular}

Table 6: Reconstruction Quality Analysis: Case 6

\begin{tabular}{|c||ccc|}
\hline CR (\%) & SNR (dB) & \\
& Modified & Standard & Improvement \\
\hline 50 & 35.52 & 31.41 & 3.10 \\
\hline 75 & 31.50 & 27.41 & 4.08 \\
\hline 87.5 & 26.85 & 22.99 & 3.86 \\
\hline 93.75 & 21.59 & 18.89 & 2.70 \\
\hline
\end{tabular}

Table 7: Reconstruction Quality Analysis: Case 7

\begin{tabular}{|c|c|c|c|}
\hline \multirow[t]{2}{*}{ CR (\%) } & \multicolumn{3}{|c|}{$\mathrm{SNR}(\mathrm{dB})$} \\
\hline & Modified & Standard & Improvement \\
\hline 50 & 37.25 & 34.50 & 2.74 \\
\hline 75 & 34.18 & 29.43 & 4.75 \\
\hline 87.5 & 29.13 & 24.73 & 4.39 \\
\hline 93.75 & 23.17 & 20.43 & 2.74 \\
\hline
\end{tabular}

Table 8: Reconstruction Quality Analysis: Case 8

\begin{tabular}{|c||ccc|}
\hline CR (\%) & SNR (dB) & \\
& Modified & Standard & Improvement \\
\hline 50 & 34.25 & 31.43 & 2.81 \\
\hline 75 & 30.79 & 26.10 & 4.69 \\
\hline 87.5 & 25.40 & 21.39 & 4.01 \\
\hline 93.75 & 19.04 & 17.36 & 1.67 \\
\hline
\end{tabular}


Table 9: Reconstruction Quality Analysis: Case 9

\begin{tabular}{|c|c|c|c|}
\hline \multirow[t]{2}{*}{ CR (\%) } & \multicolumn{3}{|c|}{ SNR (dB) } \\
\hline & Modified & Standard & Improvement \\
\hline 50 & 33.67 & 30.73 & 2.94 \\
\hline 75 & 29.16 & 25.74 & 3.41 \\
\hline 87.5 & 24.27 & 21.54 & 2.72 \\
\hline 93.75 & 19.87 & 17.85 & 2.02 \\
\hline
\end{tabular}

Table 10: Reconstruction Quality Analysis: Case 10

\begin{tabular}{|c|c|c|c|}
\hline \multirow[t]{2}{*}{ CR (\%) } & \multicolumn{3}{|c|}{$\mathrm{SNR}(\mathrm{dB})$} \\
\hline & Modified & Standard & Improvement \\
\hline 50 & 33.19 & 30.57 & 2.62 \\
\hline 75 & 30.67 & 25.50 & 5.16 \\
\hline 87.5 & 24.98 & 20.75 & 4.23 \\
\hline 93.75 & 18.65 & 16.53 & 2.12 \\
\hline
\end{tabular}

Table 11: Reconstruction Quality Analysis (Modified 2-D CS): Case 1

\begin{tabular}{|c||ccc|}
\hline CR(\%) & PSNR(dB) & \\
& Modified & Standard & Improvement \\
\hline 75 & 46.19 & 42.09 & 4.09 \\
\hline 87.5 & 40.12 & 34.23 & 5.89 \\
\hline 93.75 & 37.78 & 33.33 & 4.44 \\
\hline 96.88 & 32.33 & 29.48 & 2.85 \\
\hline
\end{tabular}

Table 12: Reconstruction Quality Analysis (Modified 2-D CS): Case 2

\begin{tabular}{|c||ccc|}
\hline CR(\%) & Modified & $\begin{array}{c}\text { PSNR(dB) } \\
\text { Standard }\end{array}$ & Improvement \\
\hline 75 & 45.22 & 40.11 & 5.11 \\
\hline 87.5 & 38.99 & 31.23 & 7.76 \\
\hline 93.75 & 36.47 & 30.19 & 6.28 \\
\hline 96.88 & 30.46 & 26.21 & 4.24 \\
\hline
\end{tabular}

Table 13: Reconstruction Quality Analysis (Modified 2-D CS): Case 3

\begin{tabular}{|c|c|c|c|}
\hline \multirow[t]{2}{*}{$\mathrm{CR}(\%)$} & \multicolumn{3}{|c|}{$\operatorname{PSNR}(\mathrm{dB})$} \\
\hline & Modified & Standard & Improvement \\
\hline 75 & 48.33 & 43.28 & 5.04 \\
\hline 87.5 & 41.27 & 34.07 & 7.21 \\
\hline 93.75 & 38.26 & 33.06 & 5.20 \\
\hline 96.88 & 32.05 & 29.03 & 3.02 \\
\hline
\end{tabular}


Table 14: Reconstruction Quality Analysis (Modified 2-D CS): Case 4

\begin{tabular}{|c||ccc|}
\hline CR(\%) & PSNR(dB) & \\
& Modified & Standard & Improvement \\
\hline 75 & 47.31 & 42.30 & 5.00 \\
\hline 87.5 & 39.97 & 33.07 & 6.89 \\
\hline 93.75 & 37.33 & 32.04 & 5.29 \\
\hline 96.88 & 31.64 & 28.08 & 3.56 \\
\hline
\end{tabular}

Table 15: Reconstruction Quality Analysis (Modified 2-D CS): Case 5

\begin{tabular}{|c|c|c|c|}
\hline \multirow[t]{2}{*}{$\mathrm{CR}(\%)$} & \multicolumn{3}{|c|}{ PSNR(dB) } \\
\hline & Modified & Standard & Improvement \\
\hline 75 & 41.09 & 38.23 & 2.85 \\
\hline 87.5 & 34.67 & 29.85 & 4.82 \\
\hline 93.75 & 32.91 & 28.84 & 4.07 \\
\hline 96.88 & 28.02 & 25.01 & 3.01 \\
\hline
\end{tabular}

Table 16: Reconstruction Quality Analysis (Modified 2-D CS): Case 6

\begin{tabular}{|c|c|c|c|}
\hline \multirow[t]{2}{*}{$\mathrm{CR}(\%)$} & \multicolumn{3}{|c|}{ PSNR(dB) } \\
\hline & Modified & Standard & Improvement \\
\hline 75 & 42.43 & 38.29 & 4.14 \\
\hline 87.5 & 36.28 & 29.71 & 6.57 \\
\hline 93.75 & 34.24 & 28.60 & 5.64 \\
\hline 96.88 & 29.30 & 24.90 & 4.40 \\
\hline
\end{tabular}

Table 17: Reconstruction Quality Analysis (Modified 2-D CS): Case 7

\begin{tabular}{|c|c|c|c|}
\hline \multirow[t]{2}{*}{$\mathrm{CR}(\%)$} & \multicolumn{3}{|c|}{$\overline{P S N R}(\mathrm{~dB})$} \\
\hline & Modified & Standard & Improvement \\
\hline 75 & 42.46 & 37.95 & 4.51 \\
\hline 87.5 & 36.99 & 29.49 & 7.49 \\
\hline 93.75 & 34.76 & 28.44 & 6.32 \\
\hline 96.88 & 29.36 & 24.75 & 4.61 \\
\hline
\end{tabular}

Table 18: Reconstruction Quality Analysis (Modified 2-D CS): Case 8

\begin{tabular}{|c|c|c|c|}
\hline \multirow[t]{2}{*}{$\mathrm{CR}(\%)$} & \multicolumn{3}{|c|}{$\operatorname{PSNR}(\mathrm{dB})$} \\
\hline & Modified & Standard & Improvement \\
\hline 75 & 46.83 & 43.08 & 3.75 \\
\hline 87.5 & 40.84 & 34.36 & 6.49 \\
\hline 93.75 & 38.45 & 33.42 & 5.03 \\
\hline 96.88 & 32.70 & 29.28 & 3.42 \\
\hline
\end{tabular}


Table 19: Reconstruction Quality Analysis (Modified 2-D CS): Case 9

\begin{tabular}{|c|c|c|c|}
\hline \multirow[t]{2}{*}{$\mathrm{CR}(\%)$} & \multicolumn{3}{|c|}{$\operatorname{PSNR}(\mathrm{dB})$} \\
\hline & Modified & Standard & Improvement \\
\hline 75 & 44.17 & 40.69 & 3.49 \\
\hline 87.5 & 37.19 & 32.09 & 5.10 \\
\hline 93.75 & 35.46 & 30.95 & 4.51 \\
\hline 96.88 & 30.93 & 27.41 & 3.51 \\
\hline
\end{tabular}

Table 20: Reconstruction Quality Analysis (Modified 2-D CS): Case 10

\begin{tabular}{|c||ccc|}
\hline CR(\%) & Modified & $\begin{array}{c}\text { PSNR(dB) } \\
\text { Standard }\end{array}$ & Improvement \\
\hline 75 & 45.53 & 41.66 & 3.87 \\
\hline 87.5 & 39.13 & 32.82 & 6.31 \\
\hline 93.75 & 36.77 & 31.92 & 4.85 \\
\hline 96.88 & 31.33 & 28.08 & 3.24 \\
\hline
\end{tabular}

Table 21: Reconstruction Quality Analysis (Modified 2-D CS with Random (Normal) Matrix): Case 1

\begin{tabular}{|c||ccc|}
\hline CR(\%) & Modified & $\begin{array}{c}\text { PSNR(dB) } \\
\text { Standard }\end{array}$ & Improvement \\
\hline 75 & $37.77 \pm 0.14$ & $35.59 \pm 0.14$ & $2.17 \pm 0.13$ \\
\hline 87.5 & $32.25 \pm 0.71$ & $29.72 \pm 0.66$ & $2.53 \pm 0.35$ \\
\hline 93.75 & $23.91 \pm 1.36$ & $22.33 \pm 1.40$ & $1.59 \pm 1.88$ \\
\hline
\end{tabular}

Table 22: Reconstruction Quality Analysis (Modified 2-D CS with Random (Normal) Matrix): Case 2

\begin{tabular}{|c||ccc|}
\hline \multicolumn{1}{|c||}{ CR(\%) } & \multicolumn{3}{|c|}{ PSNR $(\mathrm{dB})$} \\
& Modified & Standard & Improvement \\
\hline 75 & $37.47 \pm 0.15$ & $34.99 \pm 0.14$ & $2.48 \pm 0.14$ \\
\hline 87.5 & $31.38 \pm 0.72$ & $28.52 \pm 0.65$ & $2.87 \pm 0.38$ \\
\hline 93.75 & $22.71 \pm 1.40$ & $20.86 \pm 1.40$ & $1.85 \pm 1.94$ \\
\hline
\end{tabular}

Table 23: Reconstruction Quality Analysis (Modified 2-D CS with Random (Normal) Matrix): Case 3

\begin{tabular}{|c|c|c|c|}
\hline \multirow[t]{2}{*}{$\mathrm{CR}(\%)$} & \multicolumn{3}{|c|}{$\operatorname{PSNR}(\mathrm{dB})$} \\
\hline & Modified & Standard & Improvement \\
\hline 75 & $37.87 \pm 0.15$ & $35.40 \pm 0.15$ & $2.46 \pm 0.14$ \\
\hline 87.5 & $31.99 \pm 0.68$ & $29.04 \pm 0.62$ & $2.95 \pm 0.36$ \\
\hline 93.75 & $23.22 \pm 1.39$ & $21.39 \pm 1.37$ & $1.83 \pm 1.90$ \\
\hline
\end{tabular}


Table 24: Reconstruction Quality Analysis (Modified 2-D CS with Random (Normal) Matrix): Case 4

\begin{tabular}{|c|c|c|c|}
\hline \multirow[t]{2}{*}{$\mathrm{CR}(\%)$} & \multicolumn{3}{|c|}{$\operatorname{PSNR}(\mathrm{dB})$} \\
\hline & Modified & Standard & Improvement \\
\hline 75 & $37.74 \pm 0.15$ & $35.31 \pm 0.15$ & $2.43 \pm 0.14$ \\
\hline 87.5 & $31.84 \pm 0.74$ & $28.91 \pm 0.66$ & $2.93 \pm 0.39$ \\
\hline 93.75 & $23.17 \pm 1.45$ & $21.34 \pm 1.41$ & $1.83 \pm 1.96$ \\
\hline
\end{tabular}

Table 25: Reconstruction Quality Analysis (Modified 2-D CS with Random (Normal) Matrix): Case 5

\begin{tabular}{|c||ccc|}
\hline CR(\%) & PSNR $(\mathrm{dB})$ \\
& Modified & Standard & Improvement \\
\hline 75 & $37.25 \pm 0.15$ & $34.89 \pm 0.14$ & $2.36 \pm 0.13$ \\
\hline 87.5 & $31.12 \pm 0.72$ & $28.35 \pm 0.65$ & $2.77 \pm 0.38$ \\
\hline 93.75 & $22.59 \pm 1.43$ & $20.81 \pm 1.38$ & $1.78 \pm 1.93$ \\
\hline
\end{tabular}

Table 26: Reconstruction Quality Analysis (Modified 2-D CS with Random (Normal) Matrix): Case 6

\begin{tabular}{|c||ccc|}
\hline \multicolumn{1}{|c||}{ CR(\%) } & \multicolumn{3}{|c|}{ PSNR(dB) } \\
& Modified & Standard & Improvement \\
\hline 75 & $36.87 \pm 0.14$ & $34.45 \pm 0.14$ & $2.42 \pm 0.13$ \\
\hline 87.5 & $30.56 \pm 0.81$ & $27.78 \pm 0.73$ & $2.78 \pm 0.41$ \\
\hline 93.75 & $21.89 \pm 1.54$ & $20.08 \pm 1.46$ & $1.81 \pm 2.05$ \\
\hline
\end{tabular}

Table 27: Reconstruction Quality Analysis (Modified 2-D CS with Random (Normal) Matrix): Case 7

\begin{tabular}{|c||ccc|}
\hline CR(\%) & PSNR(dB) \\
& Modified & Standard & Improvement \\
\hline 75 & $36.62 \pm 0.14$ & $34.45 \pm 0.14$ & $2.42 \pm 0.13$ \\
\hline 87.5 & $30.18 \pm 0.85$ & $27.30 \pm 0.78$ & $2.88 \pm 0.43$ \\
\hline 93.75 & $21.39 \pm 1.60$ & $19.58 \pm 1.47$ & $1.82 \pm 2.09$ \\
\hline
\end{tabular}

Table 28: Reconstruction Quality Analysis (Modified 2-D CS with Random (Normal) Matrix): Case 8

\begin{tabular}{|c||ccc|}
\hline \multicolumn{1}{|c||}{ CR(\%) } & \multicolumn{3}{|c|}{ PSNR(dB) } \\
& Modified & Standard & Improvement \\
\hline 75 & $36.75 \pm 0.14$ & $34.25 \pm 0.14$ & $2.50 \pm 0.13$ \\
\hline 87.5 & $30.44 \pm 0.85$ & $27.58 \pm 0.79$ & $2.86 \pm 0.43$ \\
\hline 93.75 & $21.68 \pm 1.59$ & $19.90 \pm 1.49$ & $1.78 \pm 2.10$ \\
\hline
\end{tabular}


Table 29: Reconstruction Quality Analysis (Modified 2-D CS with Random (Normal) Matrix): Case 9

\begin{tabular}{|c||ccc|}
\hline \multicolumn{1}{|c||}{ CR(\%) } & \multicolumn{3}{c|}{ PSNR(dB) } \\
& Modified & Standard & Improvement \\
\hline 75 & $36.74 \pm 0.14$ & $34.28 \pm 0.14$ & $2.46 \pm 0.13$ \\
\hline 87.5 & $30.44 \pm 0.86$ & $27.64 \pm 0.80$ & $2.80 \pm 0.43$ \\
\hline 93.75 & $21.73 \pm 1.60$ & $19.94 \pm 1.49$ & $1.78 \pm 2.12$ \\
\hline
\end{tabular}

Table 30: Reconstruction Quality Analysis (Modified 2-D CS with Random (Normal) Matrix): Case 10

\begin{tabular}{|c||ccc|}
\hline \multicolumn{1}{|c||}{ CR(\%) } & \multicolumn{3}{c|}{ PSNR(dB) } \\
& Modified & Standard & Improvement \\
\hline 75 & $36.73 \pm 0.14$ & $34.28 \pm 0.14$ & $2.46 \pm 0.13$ \\
\hline 87.5 & $30.43 \pm 0.86$ & $27.65 \pm 0.8$ & $2.78 \pm 0.43$ \\
\hline 93.75 & $21.75 \pm 1.59$ & $19.97 \pm 1.49$ & $1.77 \pm 2.11$ \\
\hline
\end{tabular}

Table 31: Reconstruction Quality Analysis (Modified 2-D CS with Random (Bernoulli) Matrix): Case 1

\begin{tabular}{|c||ccc|}
\hline CR(\%) & \multicolumn{3}{|c|}{ PSNR(dB) } \\
& Modified & Standard & Improvement \\
\hline 75 & $30.82 \pm 0.96$ & $28.41 \pm 1.02$ & $2.41 \pm 1.41$ \\
\hline 87.5 & $26.02 \pm 1.03$ & $23.00 \pm 1.22$ & $30.2 \pm 1.60$ \\
\hline 93.75 & $19.75 \pm 1.09$ & $19.46 \pm 0.97$ & $0.29 \pm 0.32$ \\
\hline
\end{tabular}

Table 32: Reconstruction Quality Analysis (Modified 2-D CS with Random (Bernoulli) Matrix): Case 2

\begin{tabular}{|c||ccc|}
\hline CR(\%) & \multicolumn{3}{|c|}{ PSNR(dB) } \\
& Modified & Standard & Improvement \\
\hline 75 & $29.86 \pm 0.94$ & $27.66 \pm 1.08$ & $2.20 \pm 1.44$ \\
\hline 87.5 & $24.89 \pm 1.00$ & $21.80 \pm 1.21$ & $3.09 \pm 1.56$ \\
\hline 93.75 & $17.80 \pm 1.21$ & $17.45 \pm 1.07$ & $0.35 \pm 0.36$ \\
\hline
\end{tabular}

Table 33: Reconstruction Quality Analysis (Modified 2-D CS with Random (Bernoulli) Matrix): Case 3

\begin{tabular}{|c||ccc|}
\hline \multicolumn{1}{|c|}{ CR(\%) } & \multicolumn{3}{|c|}{ PSNR(dB) } \\
& Modified & Standard & Improvement \\
\hline 75 & $30.39 \pm 0.93$ & $28.17 \pm 1.07$ & $2.22 \pm 1.41$ \\
\hline 87.5 & $25.35 \pm 1.02$ & $22.20 \pm 1.20$ & $3.15 \pm 1.57$ \\
\hline 93.75 & $18.47 \pm 1.33$ & $18.09 \pm 1.16$ & $0.37 \pm 0.39$ \\
\hline
\end{tabular}


Table 34: Reconstruction Quality Analysis (Modified 2-D CS with Random (Bernoulli) Matrix): Case 4

\begin{tabular}{|c||ccc|}
\hline \multicolumn{1}{|c||}{ CR(\%) } & \multicolumn{3}{|c|}{ PSNR(dB) } \\
& Modified & Standard & Improvement \\
\hline 75 & $30.34 \pm 0.91$ & $27.89 \pm 1.06$ & $2.45 \pm 1.38$ \\
\hline 87.5 & $25.30 \pm 1.01$ & $22.25 \pm 1.20$ & $3.05 \pm 1.57$ \\
\hline 93.75 & $14.14 \pm 1.31$ & $17.79 \pm 1.16$ & $0.35 \pm 0.39$ \\
\hline
\end{tabular}

Table 35: Reconstruction Quality Analysis (Modified 2-D CS with Random (Bernoulli) Matrix): Case 5

\begin{tabular}{|c||ccc|}
\hline CR(\%) & PSNR $(\mathrm{dB})$ \\
& Modified & Standard & Improvement \\
\hline 75 & $29.49 \pm 0.90$ & $27.22 \pm 1.06$ & $2.27 \pm 1.38$ \\
\hline 87.5 & $24.63 \pm 1.01$ & $21.64 \pm 1.21$ & $2.99 \pm 1.57$ \\
\hline 93.75 & $17.59 \pm 1.35$ & $17.24 \pm 1.20$ & $0.34 \pm 0.39$ \\
\hline
\end{tabular}

Table 36: Reconstruction Quality Analysis (Modified 2-D CS with Random (Bernoulli) Matrix): Case 6

\begin{tabular}{|c||ccc|}
\hline \multicolumn{1}{|c||}{ CR(\%) } & \multicolumn{3}{c|}{ PSNR(dB) } \\
& Modified & Standard & Improvement \\
\hline 75 & $28.84 \pm 0.87$ & $26.54 \pm 1.04$ & $2.30 \pm 1.34$ \\
\hline 87.5 & $24.20 \pm 1.00$ & $21.16 \pm 1.20$ & $30.4 \pm 1.55$ \\
\hline 93.75 & $16.69 \pm 1.29$ & $16.37 \pm 1.15$ & $0.32 \pm 0.37$ \\
\hline
\end{tabular}

Table 37: Reconstruction Quality Analysis (Modified 2-D CS with Random (Bernoulli) Matrix): Case 7

\begin{tabular}{|c||ccc|}
\hline CR(\%) & PSNR(dB) \\
& Modified & Standard & Improvement \\
\hline 75 & $28.30 \pm 0.86$ & $25.93 \pm 1.04$ & $2.37 \pm 1.34$ \\
\hline 87.5 & $23.87 \pm 0.99$ & $20.74 \pm 1.20$ & $3.13 \pm 1.54$ \\
\hline 93.75 & $15.91 \pm 1.29$ & $15.61 \pm 1.15$ & $0.30 \pm 0.38$ \\
\hline
\end{tabular}

Table 38: Reconstruction Quality Analysis (Modified 2-D CS with Random (Bernoulli) Matrix): Case 8

\begin{tabular}{|c|c|c|c|}
\hline \multirow[t]{2}{*}{$\mathrm{CR}(\%)$} & \multicolumn{3}{|c|}{$\operatorname{PSNR}(\mathrm{dB})$} \\
\hline & Modified & Standard & Improvement \\
\hline 75 & $28.57 \pm 0.87$ & $26.15 \pm 1.04$ & $2.42 \pm 1.35$ \\
\hline 87.5 & $24.07 \pm 1.00$ & $21.05 \pm 1.20$ & $3.02 \pm 1.55$ \\
\hline 93.75 & $16.21 \pm 1.29$ & $15.91 \pm 1.15$ & $0.3 \pm 0.38$ \\
\hline
\end{tabular}


Table 39: Reconstruction Quality Analysis (Modified 2-D CS with Random (Bernoulli) Matrix): Case 9

\begin{tabular}{|c||ccc|}
\hline \multicolumn{1}{|c||}{ CR(\%) } & \multicolumn{3}{|c|}{ PSNR(dB) } \\
& Modified & Standard & Improvement \\
\hline 75 & $28.58 \pm 0.87$ & $26.18 \pm 1.04$ & $2.40 \pm 1.34$ \\
\hline 87.5 & $24.11 \pm 0.98$ & $21.14 \pm 1.19$ & $2.98 \pm 1.54$ \\
\hline 93.75 & $16.20 \pm 1.28$ & $15.91 \pm 1.15$ & $0.30 \pm 0.38$ \\
\hline
\end{tabular}

Table 40: Reconstruction Quality Analysis (Modified 2-D CS with Random (Bernoulli) Matrix): Case 10

\begin{tabular}{|c||ccc|}
\hline \multicolumn{1}{|c||}{ CR(\%) } & \multicolumn{3}{c|}{ PSNR(dB) } \\
& Modified & Standard & Improvement \\
\hline 75 & $28.51 \pm 0.86$ & $26.20 \pm 1.04$ & $2.31 \pm 1.34$ \\
\hline 87.5 & $24.15 \pm 0.99$ & $21.15 \pm 1.20$ & $3.00 \pm 1.55$ \\
\hline 93.75 & $16.30 \pm 1.26$ & $16.01 \pm 1.12$ & $0.29 \pm 0.38$ \\
\hline
\end{tabular}




\section{References}

[1] C. E. Shannon, "A mathematical theory of communication," The Bell System Technical Journal, vol. 27, pp. 379-423, July 1948.

[2] D. L. Donoho, "Compressed sensing," IEEE Transactions on Information Theory, vol. 52, pp. 1289-1306, April 2006.

[3] R. G. Baraniuk, "Compressive sensing [lecture notes]," IEEE Signal Processing Magazine, vol. 24, pp. 118-121, July 2007.

[4] M. Aharon, M. Elad, and A. Bruckstein, "rmK-SVD: An algorithm for designing overcomplete dictionaries for sparse representation," IEEE Transactions on Signal Processing, vol. 54, pp. 4311-4322, Nov 2006.

[5] Z. Li, S. Ding, and Y. Li, "A fast algorithm for learning overcomplete dictionary for sparse representation based on proximal operators," Neural Computation, vol. 27, no. 9, pp. 1951-1982, 2015.

[6] X. Chen, Z. Du, J. Li, X. Li, and H. Zhang, "Compressed sensing based on dictionary learning for extracting impulse components," Signal Processing, vol. 96, no. Part A, pp. 94-109, 2014.

[7] G. Peyre, "Best basis compressed sensing," IEEE Transactions on Signal Processing, vol. 58, pp. 2613-2622, May 2010. 
[8] W. Dong, L. Zhang, G. Shi, and X. Wu, "Image deblurring and super-resolution by adaptive sparse domain selection and adaptive regularization," IEEE Transactions on Image Processing, vol. 20, pp. 1838-1857, July 2011.

[9] A. Krause and V. Cevher, "Submodular dictionary selection for sparse representation," in Proceedings of the 27th International Conference on International Conference on Machine Learning, ICML'10, (USA), pp. 567-574, Omnipress, 2010.

[10] L. Zelnik-Manor, K. Rosenblum, and Y. C. Eldar, "Dictionary optimization for block-sparse representations," IEEE Transactions on Signal Processing, vol. 60, pp. 2386-2395, May 2012.

[11] E. J. Candès, "Theory of signals/mathematical analysis," Comptes rendus Mathematique, vol. 346, no. 9-10, pp. 589-592, 2008.

[12] M. Elad, Sparse and Redundant Representations: From Theory to Applications in Signal and Image Processing. Springer Publishing Company, Incorporated, 1st ed., 2010.

[13] D. L. Donoho and X. Huo, "Uncertainty principles and ideal atomic decomposition," IEEE Transactions on Information Theory, vol. 47, no. 7, pp. 2845 2862, 2001.

[14] L. C. Potter, E. Ertin, J. T. Parker, and M. Cetin, "Sparsity and compressed sensing in radar imaging," Proceedings of the IEEE, vol. 98, pp. 1006-1020, June 2010. 
[15] Y. Arjoune, N. Kaabouch, H. El Ghazi, and A. Tamtaoui, "A performance comparison of measurement matrices in compressive sensing," International Journal of Communication Systems, vol. 31, no. 10, pp. 1-9, 2018.

[16] R. Baraniuk, M. Davenport, R. DeVore, and M. Wakin, "A simple proof of the restricted isometry property for random matrices," Constructive Approximation, vol. 28, pp. 253-263, Dec 2008.

[17] E. J. Candès, "The restricted isometry property and its implications for compressed sensing," Comptes Rendus Mathematique, vol. 346, no. 9, pp. 589 - 592, 2008.

[18] R. van Handel, "Structured random matrices," in Convexity and Concentration, pp. 107-156, Springer New York, 2017.

[19] H. L. Yap, M. B. Wakin, and C. J. Rozell, "Stable manifold embeddings with structured random matrices," IEEE Journal of Selected Topics in Signal Processing, vol. 7, pp. 720-730, Aug 2013.

[20] W. U. Bajwa, J. D. Haupt, G. M. Raz, S. J. Wright, and R. D. Nowak, "Toeplitzstructured compressed sensing matrices," in 2007 IEEE/SP 14th Workshop on Statistical Signal Processing, pp. 294-298, Aug 2007.

[21] G. E. Pfander, H. Rauhut, and J. A. Tropp, "The restricted isometry property for time-frequency structured random matrices," Probability Theory and Related Fields, vol. 156, pp. 707-737, Aug 2013.

[22] H. Rauhut, "Compressive sensing and structured random matrices," Theoretical Foundations and Numerical Methods for Sparse Recovery, 2010. 
[23] K. Życzkowski, K. A. Penson, I. Nechita, and B. Collins, "Generating random density matrices," Journal of Mathematical Physics, vol. 52, no. 6, pp. 062201(1-20), 2011.

[24] J. Aljadeff, D. Renfrew, and M. Stern, "Eigenvalues of block structured asymmetric random matrices," Journal of Mathematical Physics, vol. 56, no. 10, pp. 103502-(1-14), 2015.

[25] H. Rauhut, J. Romberg, and J. A. Tropp, "Restricted isometries for partial random circulant matrices," Applied and Computational Harmonic Analysis, vol. 32 , no. 2, pp. $242-254,2012$.

[26] L. Yu, J.-P. Barbot, G. Zheng, and H. Sun, "Toeplitz-structured chaotic sensing matrix for compressive sensing," in IEEE, IET International Symposium on Communication Systems, Networks and Digital Signal Processing, (Newcastle, United Kingdom), Oct. 2010.

[27] K. Li, L. Gan, and C. Ling, "Convolutional compressed sensing using deterministic sequences," IEEE Transactions on Signal Processing, vol. 61, pp. 740-752, Feb 2013.

[28] R. R. Naidu, P. Jampana, and C. S. Sastry, "Deterministic compressed sensing matrices: Construction via euler squares and applications," IEEE Transactions on Signal Processing, vol. 64, pp. 3566-3575, July 2016.

[29] L. Applebaum, S. D. Howard, S. Searle, and R. Calderbank, "Chirp sensing codes: Deterministic compressed sensing measurements for fast recovery," $A p$ plied and Computational Harmonic Analysis, vol. 26, no. 2, pp. 283 - 290, 2009. 
[30] S. D. Howard, A. R. Calderbank, and S. J. Searle, "A fast reconstruction algorithm for deterministic compressive sensing using second order reed-muller codes," in 2008 42nd Annual Conference on Information Sciences and Systems, pp. 11-15, March 2008.

[31] N. Ailon and E. Liberty, "Fast dimension reduction using rademacher series on dual BCH codes," Discrete \& Computational Geometry, vol. 42, p. 615, Sep 2008.

[32] R. Calderbank, S. Howard, and S. Jafarpour, "Construction of a large class of deterministic sensing matrices that satisfy a statistical isometry property," IEEE Journal of Selected Topics in Signal Processing, vol. 4, pp. 358-374, April 2010

[33] A. Amini and F. Marvasti, "Deterministic construction of binary, bipolar, and ternary compressed sensing matrices," IEEE Transactions on Information Theory, vol. 57, pp. 2360-2370, April 2011.

[34] A. Amini, V. Montazerhodjat, and F. Marvasti, "Matrices with small coherence using p-ary block codes," IEEE Transactions on Signal Processing, vol. 60, pp. 172-181, Jan 2012.

[35] A. Ravelomanantsoa, H. Rabah, and A. Rouane, "Compressed sensing: A simple deterministic measurement matrix and a fast recovery algorithm," IEEE Transactions on Instrumentation and Measurement, vol. 64, pp. 3405-3413, Dec 2015.

[36] E. J. Candès, J. Romberg, and T. Tao, "Robust uncertainty principles: exact signal reconstruction from highly incomplete frequency information," IEEE Transactions on Information Theory, vol. 52, pp. 489-509, Feb 2006. 
[37] S. S. Chen, D. L. Donoho, and M. A. Saunders, "Atomic decomposition by basis pursuit," Society for Industrial and Applied Mathematics, vol. 43, pp. 129-159, Mar 2001.

[38] R. Tibshirani, "Regression shrinkage and selection via the LASSO," Journal of the Royal Statistical Society, vol. 58, no. 1, pp. 267-288, 1996.

[39] M. A. T. Figueiredo, R. D. Nowak, and S. J. Wright, "Gradient projection for sparse reconstruction: Application to compressed sensing and other inverse problems," IEEE Journal of Selected Topics in Signal Processing, vol. 1, pp. 586-597, Dec 2007.

[40] Y. C. Pati, R. Rezaiifar, and P. S. Krishnaprasad, "Orthogonal matching pursuit: recursive function approximation with applications to wavelet decomposition," in Proceedings of 27th Asilomar Conference on Signals, Systems and Computers, pp. 40-44 vol.1, Nov 1993.

[41] J. A. Tropp and A. C. Gilbert, "Signal recovery from random measurements via orthogonal matching pursuit," IEEE Transactions on Information Theory, vol. 53, pp. 4655-4666, Dec 2007.

[42] D. Needell and R. Vershynin, "Uniform uncertainty principle and signal recovery via regularized orthogonal matching pursuit," Foundations of Computational Mathematics, vol. 9, pp. 317-334, Jun 2009.

[43] D. Needell and J. Tropp, "CoSaMP: Iterative signal recovery from incomplete and inaccurate samples," Applied and Computational Harmonic Analysis, vol. 26, no. 3, pp. $301-321,2009$. 
[44] D. L. Donoho, Y. Tsaig, I. Drori, and J. luc Starck, "Sparse solution of underdetermined linear equations by stagewise orthogonal matching pursuit, submitted to," IEEE Transactions on Information theory, 2006.

[45] E. Crespo Marques, N. Maciel, L. Naviner, H. Cai, and J. Yang, "A review of sparse recovery algorithms," IEEE Access, vol. 7, pp. 1300-1322, 2019.

[46] H. Mohimani, M. Babaie-Zadeh, and C. Jutten, "A fast approach for overcomplete sparse decomposition based on smoothed $\ell^{0}$ norm," IEEE Transactions on Signal Processing, vol. 57, pp. 289-301, Jan 2009.

[47] M. F. Duarte and R. G. Baraniuk, "Kronecker compressive sensing," IEEE Transactions on Image Processing, vol. 21, pp. 494-504, Feb 2012.

[48] M. F. Duarte and R. G. Baraniuk, "Kronecker product matrices for compressive sensing," Rice University, Department of Electrical and Computer Engineering, Houston, TX, Tech. Rep. TREE-1105, March 2011.

[49] H. Zanddizari, S. Rajan, and H. Zarrabi, "Increasing the quality of reconstructed signal in compressive sensing utilizing kronecker technique," Biomedical Engineering Letters, vol. 8, pp. 239-247, May 2018.

[50] D. Mitra, H. Zanddizari, and S. Rajan, "Improvement of signal quality during recovery of compressively sensed ECG signals," in 2018 IEEE International Symposium on Medical Measurements and Applications (MeMeA), pp. 1-5, June 2018.

[51] D. Mitra, H. Zanddizari, and S. Rajan, "Investigation of kronecker-based recovery of compressed ECG measurements," IEEE Transactions on Instrumentation and Measurement, 2019. (Under Correction). 
[52] "MIT-BIH Arrhythmia Database." Available: http://www.physionet.org/ physiobank/database/mitdb/. [Online].

[53] "World health statistics 2018." Available: https://apps.who.int/iris/ bitstream/handle/10665/272596/9789241565585-eng.pdf?ua=1. [Online].

[54] C. D. Capua, A. Meduri, and R. Morello, "A smart ECG measurement system based on web-service-oriented architecture for telemedicine applications," IEEE Transactions on Instrumentation and Measurement, vol. 59, pp. 2530-2538, Oct 2010.

[55] L. Fanucci, S. Saponara, T. Bacchillone, M. Donati, P. Barba, I. Sanchez-Tato, and C. Carmona, "Sensing devices and sensor signal processing for remote monitoring of vital signs in CHF patients," IEEE Transactions on Instrumentation and Measurement, vol. 62, pp. 553-569, March 2013.

[56] C. Park, P. H. Chou, Y. Bai, R. Matthews, and A. Hibbs, "An ultra-wearable, wireless, low power ECG monitoring system," in 2006 IEEE Biomedical Circuits and Systems Conference, pp. 241-244, Nov 2006.

[57] Y. Chuo, M. Marzencki, B. Hung, C. Jaggernauth, K. Tavakolian, P. Lin, and B. Kaminska, "Mechanically flexible wireless multisensor platform for human physical activity and vitals monitoring," IEEE Transactions on Biomedical Circuits and Systems, vol. 4, pp. 281-294, Oct 2010.

[58] E. Nemati, M. J. Deen, and T. Mondal, "A wireless wearable ECG sensor for long-term applications," IEEE Communications Magazine, vol. 50, pp. 36-43, January 2012. 
[59] S. Movassaghi, M. Abolhasan, J. Lipman, D. Smith, and A. Jamalipour, "Wireless body area networks: A survey," IEEE Communications Surveys Tutorials, vol. 16, no. 3, pp. 1658-1686, 2014.

[60] J. Zhang, Z. L. Yu, Z. Gu, Y. Li, and Z. Lin, "Multichannel electrocardiogram reconstruction in wireless body sensor networks through weighted $\ell_{1,2}$ minimization," IEEE Transactions on Instrumentation and Measurement, vol. 67, pp. 2024-2034, Sep. 2018.

[61] R. Yan, H. Sun, and Y. Qian, "Energy-aware sensor node design with its application in wireless sensor networks," IEEE Transactions on Instrumentation and Measurement, vol. 62, pp. 1183-1191, May 2013.

[62] H. Cao, V. Leung, C. Chow, and H. Chan, "Enabling technologies for wireless body area networks: A survey and outlook," IEEE Communications Magazine, vol. 47, pp. 84-93, Dec 2009.

[63] A. Kadrolkar, R. X. Gao, R. Yan, and W. Gong, "Variable-word-length coding for energy-aware signal transmission," IEEE Transactions on Instrumentation and Measurement, vol. 61, pp. 850-864, April 2012.

[64] S. Aviyente, "Compressed sensing framework for EEG compression," in 2007 IEEE/SP 14th Workshop on Statistical Signal Processing, pp. 181-184, Aug 2007.

[65] S. Şenay, L. F. Chaparro, M. Sun, and R. J. Sclabassi, "Compressive sensing and random filtering of EEG signals using slepian basis," in 2008 16th European Signal Processing Conference, pp. 1-5, Aug 2008. 
[66] P. R. Muduli and A. Mukherjee, "A subspace projection-based joint sparse recovery method for structured biomedical signals," IEEE Transactions on Instrumentation and Measurement, vol. 66, pp. 234-242, Feb 2017.

[67] S. M. S. Jalaleddine, C. G. Hutchens, R. D. Strattan, and W. A. Coberly, "ECG data compression techniques-a unified approach," IEEE Transactions on Biomedical Engineering, vol. 37, pp. 329-343, April 1990.

[68] C. Ku, K. Hung, T. Wu, and H. Wang, "Wavelet-based ECG data compression system with linear quality control scheme," IEEE Transactions on Biomedical Engineering, vol. 57, pp. 1399-1409, June 2010.

[69] H.-H. Chou, Y.-J. Chen, Y.-C. Shiau, and T. son Kuo, "An effective and efficient compression algorithm for ECG signals with irregular periods," IEEE Transactions on Biomedical Engineering, vol. 53, pp. 1198-1205, June 2006.

[70] H. Mamaghanian, N. Khaled, D. Atienza, and P. Vandergheynst, "Compressed sensing for real-time energy-efficient ECG compression on wireless body sensor nodes," IEEE Transactions on Biomedical Engineering, vol. 58, pp. 2456-2466, Sept 2011.

[71] L. F. Polania, R. E. Carrillo, M. Blanco-Velasco, and K. E. Barner, "Compressed sensing based method for ECG compression," in 2011 IEEE International Conference on Acoustics, Speech and Signal Processing (ICASSP), pp. 761-764, May 2011.

[72] W. Yan, Q. Wang, Y. Shen, Y. Wang, and Q. Han, "An efficient data gathering and reconstruction method in WSNs based on compressive sensing," in 2012 IEEE International Instrumentation and Measurement Technology Conference Proceedings, pp. 2028-2033, May 2012. 
[73] A. M. R. Dixon, E. G. Allstot, D. Gangopadhyay, and D. J. Allstot, "Compressed sensing system considerations for ECG and EMG wireless biosensors," IEEE Transactions on Biomedical Circuits and Systems, vol. 6, pp. 156-166, April 2012.

[74] K. Kanoun, H. Mamaghanian, N. Khaled, and D. Atienza, "A real-time compressed sensing-based personal electrocardiogram monitoring system," in 2011 Design, Automation Test in Europe, pp. 1-6, March 2011.

[75] D. Craven, B. McGinley, L. Kilmartin, M. Glavin, and E. Jones, "Compressed sensing for bioelectric signals: A review," IEEE Journal of Biomedical and Health Informatics, vol. 19, pp. 529-540, March 2015.

[76] G. Da Poian, R. Bernardini, and R. Rinaldo, "Separation and analysis of fetalECG signals from compressed sensed abdominal ECG recordings," IEEE Transactions on Biomedical Engineering, vol. 63, pp. 1269-1279, June 2016.

[77] G. Da Poian, C. J. Rozell, R. Bernardini, R. Rinaldo, and G. D. Clifford, "Matched filtering for heart rate estimation on compressive sensing ECG measurements," IEEE Transactions on Biomedical Engineering, vol. 65, pp. 1349 1358, June 2018.

[78] C. Chung, D. Lee, W. Luk, and J. D. Villasenor, "Hardware generation of arbitrary random number distributions from uniform distributions via the inversion method," IEEE Transactions on Very Large Scale Integration (VLSI) Systems, vol. 15, pp. 952 - 962, Aug 2007.

[79] S. Raj and K. C. Ray, "ECG signal analysis using dct-based dost and pso optimized svm," IEEE Transactions on Instrumentation and Measurement, vol. 66, pp. 470-478, March 2017. 
[80] R. G. Baraniuk, V. Cevher, M. F. Duarte, and C. Hegde, "Model-based compressive sensing," IEEE Transactions on Information Theory, vol. 56, pp. 19822001, April 2010.

[81] M. S. Crouse, R. D. Nowak, and R. G. Baraniuk, "Wavelet-based statistical signal processing using hidden markov models," IEEE Transactions on Signal Processing, vol. 46, pp. 886-902, April 1998.

[82] Y. Zigel, A. Cohen, and A. Katz, "The weighted diagnostic distortion (WDD) measure for ECG signal compression," IEEE Transactions on Biomedical Engineering, vol. 47, pp. 1422-1430, Nov 2000.

[83] M. Ishijima, "Fundamentals of the decision of optimum factors in the ECG data compression (special section on ECG data compression)," IEICE transactions on information and systems, vol. 76, pp. 1398-1403, dec 1993.

[84] C. M. Fira and L. Goras, "An ECG signals compression method and its validation using nns," IEEE Transactions on Biomedical Engineering, vol. 55, pp. 1319-1326, April 2008.

[85] A. Al-Fahoum, "Quality assessment of ECG compression techniques using a wavelet-based diagnostic measure," IEEE Transactions on Information Technology in Biomedicine, vol. 10, no. 1, pp. 182 - 191, 2006.

[86] "MIT-BIH Arrhythmia Database Directory." Available: https://physionet. org/physiobank/database/html/mitdbdir/intro.htm. [Online].

[87] G. B. Moody and R. G. Mark, "The impact of the MIT-BIH Arrhythmia Database," IEEE Engineering in Medicine and Biology Magazine, vol. 20, pp. 45-50, May 2001. 
[88] D. Mitra, H. Zanddizari, and S. Rajan, "Improvement of recovery in segmentation-based parallel compressive sensing," in 2018 IEEE International Symposium on Signal Processing and Information Technology (ISSPIT), pp. 501-506, Dec 2018.

[89] "National center for image-guided therapy, harvard medical school." Available: http://2014.ncigt.org/publications/item/view/541. [Online].

[90] B. Han, F. Wu, and D. Wu, "Image representation by compressed sensing," in 2008 15th IEEE International Conference on Image Processing, pp. 1344-1347, Oct 2008 .

[91] S. Mun and J. E. Fowler, "Block compressed sensing of images using directional transforms," in 2009 16th IEEE International Conference on Image Processing (ICIP), pp. 3021-3024, Nov 2009.

[92] L. Gan, "Block compressed sensing of natural images," in 2007 15th International Conference on Digital Signal Processing, pp. 403-406, July 2007.

[93] L. Gan, T. T. Do, and T. D. Tran, "Fast compressive imaging using scrambled block hadamard ensemble," in 2008 16th European Signal Processing Conference, pp. 1-5, Aug 2008.

[94] G. Coluccia, S. K. Kuiteing, A. Abrardo, M. Barni, and E. Magli, "Progressive compressed sensing and reconstruction of multidimensional signals using hybrid transform/prediction sparsity model," IEEE Journal on Emerging and Selected Topics in Circuits and Systems, vol. 2, pp. 340-352, Sep. 2012. 
[95] S. F. Cotter, B. D. Rao, and K. Kreutz-Delgado, "Sparse solutions to linear inverse problems with multiple measurement vectors," IEEE Transactions on Signal Processing, vol. 53, pp. 2477-2488, July 2005.

[96] S. Ujan, S. Ghorshi, M. Pourebrahim, and S. A. Khoshnevis, "On the use of compressive sensing for image enhancement," in 2016 UKSim-AMSS 18th International Conference on Computer Modelling and Simulation (UKSim), pp. 167171, April 2016.

[97] "Mathworks ${ }^{\circledR}$, (2013). image processing toolbox ${ }^{\text {TM }}$." Retrived from: https : //www.mathworks. com/help/images/index.html. [Online].

[98] Z. Wang, A. C. Bovik, H. R. Sheikh, and E. P. Simoncelli, "Image quality assessment: from error visibility to structural similarity," IEEE Transactions on Image Processing, vol. 13, pp. 600-612, April 2004.

[99] "MathWorks ${ }^{\circledR}$ Documentation on SSIM." Available: https://www . mathworks . com/help/images/ref/ssim.html\#bt5rkbx-1-DynamicRange. [Online].

[100] A. Fedorov, R. Beichel, J. Kalpathy-Cramer, J. Finet, J.-C. Fillion-Robin, S. Pujol, C. Bauer, D. Jennings, F. Fennessy, M. Sonka, J. Buatti, S. Aylward, J. V. Miller, S. Pieper, and R. Kikinis, "3D slicer as an image computing platform for the quantitative imaging network," Magnetic Resonance Imaging, vol. 30, no. 9, pp. 1323 - 1341, 2012.

[101] A. Hore and D. Ziou, "Image quality metrics: Psnr vs. ssim," in 2010 20th International Conference on Pattern Recognition, pp. 2366-2369, Aug 2010. 
[102] D. Mitra, S. Rajan, and B. Balaji, "A deterministic compressive sensing approach for compressed domain image analysis," in 2019 IEEE Sensors Applications Symposium (SAS), pp. 1-6, March 2019.

[103] H. Sadreazami, D. Mitra, S. Rajan, and M. Bolic, "Fall detection in compressed domain using machine learning," 2019, (Under Preparation).

[104] X. Wu, W. Dong, X. Zhang, and G. Shi, "Model-assisted adaptive recovery of compressed sensing with imaging applications," IEEE Transactions on Image Processing, vol. 21, pp. 451-458, Feb 2012.

[105] X. Liu, D. Zhai, J. Zhou, X. Zhang, D. Zhao, and W. Gao, "Compressive sampling-based image coding for resource-deficient visual communication," IEEE Transactions on Image Processing, vol. 25, pp. 2844-2855, June 2016.

[106] N. Eslahi, A. Aghagolzadeh, and S. M. H. Andargoli, "Image/video compressive sensing recovery using joint adaptive sparsity measure," Neurocomputing, vol. 200 , pp. 88 - 109, 2016.

[107] E. Lagunas, S. K. Sharma, S. Chatzinotas, and B. Ottersten, "Compressive sensing based energy detector," in 2016 24th European Signal Processing Conference (EUSIPCO), pp. 1678-1682, Aug 2016.

[108] K. Zhang, L. Zhang, and M. Yang, "Fast compressive tracking," IEEE Transactions on Pattern Analysis and Machine Intelligence, vol. 36, pp. 2002-2015, Oct 2014 .

[109] V. Singhal, A. Majumdar, and R. K. Ward, "Semi-supervised deep blind compressed sensing for analysis and reconstruction of biomedical signals from compressive measurements," IEEE Access, vol. 6, pp. 545-553, 2018. 
[110] A. Baumberg, "Reliable feature matching across widely separated views," in Proceedings IEEE Conference on Computer Vision and Pattern Recognition. CVPR 2000 (Cat. No.PR00662), vol. 1, pp. 774-781 vol.1, June 2000.

[111] T. Lindeberg, "Feature detection with automatic scale selection," International Journal of Computer Vision, vol. 30, pp. 79-116, Nov 1998.

[112] K. Mikolajczyk and C. Schmid, "An affine invariant interest point detector," in Computer Vision - ECCV 2002 (A. Heyden, G. Sparr, M. Nielsen, and P. Johansen, eds.), (Berlin, Heidelberg), pp. 128-142, Springer Berlin Heidelberg, 2002.

[113] K. Mikolajczyk and C. Schmid, "Scale \& affine invariant interest point detectors," International Journal of Computer Vision, vol. 60, pp. 63-86, Oct 2004.

[114] D. G. Lowe, "Distinctive image features from scale-invariant keypoints," International Journal of Computer Vision, vol. 60, pp. 91-110, Nov 2004.

[115] H. Bay, A. Ess, T. Tuytelaars, and L. V. Gool, "Speeded-up robust features (surf)," Computer Vision and Image Understanding, vol. 110, no. 3, pp. 346 359, 2008. Similarity Matching in Computer Vision and Multimedia.

[116] K. Mikolajczyk and C. Schmid, "A performance evaluation of local descriptors," IEEE Transactions on Pattern Analysis and Machine Intelligence, vol. 27, pp. 1615-1630, Oct. 2005.

[117] J. Svab, T. Krajnik, J. Faigl, and L. Preucil, "Fpga based speeded up robust features," in 2009 IEEE International Conference on Technologies for Practical Robot Applications, pp. 35-41, Nov 2009. 
[118] D. Fleck and Z. Duric, "Affine invariant-based classification of inliers and outliers for image matching," in Image Analysis and Recognition (M. Kamel and A. Campilho, eds.), (Berlin, Heidelberg), pp. 268-277, Springer Berlin Heidelberg, 2009.

[119] P. Torr and A. Zisserman, "MLESAC: A new robust estimator with application to estimating image geometry," Computer Vision and Image Understanding, vol. 78 , no. 1 , pp. $138-156,2000$.

[120] B. Bhashyam, R. Sithiravel, Z. Daya, and K. Thiagalingam, "Aspects of detection and tracking of ground targets from an airborne EO/IR sensor," in Proc. SPIE Signal Processing, Sensor/Information Fusion, and Target Recognition XXIV (M. Kamel and A. Campilho, eds.), (Baltimore, United States), SPIE $9474,2015$.

[121] "LANDSAT medium resolution image courtesy of the u.s. geological survey." Available: https://usgs.gov. [Online].

[122] J. A. Barsi, K. Lee, G. Kvaran, B. L. Markham, and J. A. Pedelty, "The spectral response of the Landsat-8 operational land imager," Remote Sensing, vol. 6, no. 10, pp. 10232-10251, 2014.

[123] "Worldview-2 earth observation satellite, USA." Available: https://www. digitalglobe.com/about/our-constellation. [Online].

[124] G. H. Golub and C. F. V. Loan, Matrix Computations. MD: Johns Hopkins, 3rd ed., 1996. 